\title{
Development of constant-time correlated spectroscopy on a 3T clinical MRI/MRS scanner
}

\author{
Srilatha Ainala \\ West Virginia University
}

Follow this and additional works at: https://researchrepository.wvu.edu/etd

\section{Recommended Citation}

Ainala, Srilatha, "Development of constant-time correlated spectroscopy on a 3T clinical MRI/MRS scanner" (2005). Graduate Theses, Dissertations, and Problem Reports. 1877.

https://researchrepository.wvu.edu/etd/1877

This Thesis is protected by copyright and/or related rights. It has been brought to you by the The Research Repository @ WVU with permission from the rights-holder(s). You are free to use this Thesis in any way that is permitted by the copyright and related rights legislation that applies to your use. For other uses you must obtain permission from the rights-holder(s) directly, unless additional rights are indicated by a Creative Commons license in the record and/ or on the work itself. This Thesis has been accepted for inclusion in WVU Graduate Theses, Dissertations, and Problem Reports collection by an authorized administrator of The Research Repository @ WVU. For more information, please contact researchrepository@mail.wvu.edu. 
Development of Constant-Time Correlated Spectroscopy on a 3T clinical MRI/MRS Scanner

\author{
Srilatha Ainala
}

Thesis submitted to the

College of Engineering and Mineral Resources

at West Virginia University

in partial fulfillment of the requirements

for the degree of

\author{
Master of Science \\ in \\ Electrical Engineering
}

Dr. Wils L. Cooley Ph.D., Chair

Dr. Mark A. Jerabek Ph.D.

Dr. Matthew C. Valenti Ph.D.

Dr. S. Sendhil Velan Ph.D.

Dr. Raymond R. Raylman Ph.D.

Lane Department of Computer Science and Electrical Engineering

Center for Advanced Imaging, WVU School of Medicine

\author{
Morgantown, West Virginia \\ 2005
}

Keywords: MRS, L-COSY, CT-COSY.

Copyright 2005 Srilatha Ainala 


\title{
ABSTRACT \\ Development of Constant-Time Correlated Spectroscopy on a 3T clinical MRI/MRS Scanner
}

\author{
Srilatha Ainala
}

Magnetic Resonance Spectroscopy (MRS) is widely used both as a fundamental research technique, as well as diagnostic tool in medical research providing unique information about the metabolites. In this thesis, we report our work on the development of novel localized constant-time correlated Magnetic Resonance Spectroscopic technique for resolving the overlapping metabolites and also to improve the quantification strategies. The new sequence was implemented on a GE 3T MRI/MRS scanner. The underlying spin dynamics is discussed for a two-spin $1 / 2$ system with a $J$ coupling. We demonstrate the coherence transfer to cross peaks and diagonal peaks by changing the constant time. Theoretical simulations of the MR spectra for simple spin systems were performed and for our new sequence using GAMMA program. In addition we have also implemented theoretical simulations of one and two-dimensional MRS sequences within GAMMA environment for understanding the effect of field strength on MR spectra and also $J$ modulation. 


\section{Dedication}

This thesis is dedicated to my uncle Late Narsimha Reddy and aunt Vimala for providing unlimited love, guidance, support and enthusiasm throughout my life. I also dedicate this thesis work to my parents for their constant support, guidance and their help to fulfill my dreams in pursuing my masters. I also thank my sisters and brother for their love and affection.

And above all to my fiancé Ramgopal Reddy who has been my great source of inspiration and motivation. 


\section{Acknowledgements}

I would like to express my sincere thanks to Dr. Sendhil Velan, for giving me the opportunity to work under him and finish my thesis under his guidance. Also special thanks to Dr. Raylman Raymond for his help in finding this job and also for his encouragement in my work.

I also wish to thank Dr. Mark Jerabek, Dr. Wils Cooley my committee members, for their valuable guidance, suggestions, comments. I am indebted to Dr. Mattew Valenti for being on my committee and giving valuable comments.

I gratefully acknowledge my friend Senthil Ramamurthy for giving me extreme support and encouragement in the project.

Finally I would like to thank the members of Center for Advanced Imaging / Department of Radiology for all their timely support and guidance. 


\section{Table of Contents}

Chapter 1

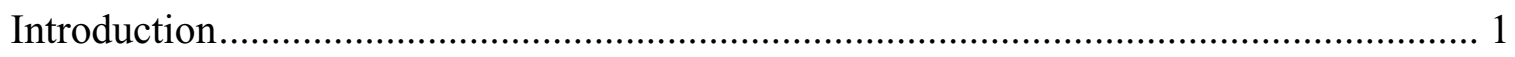

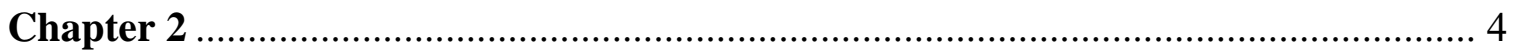

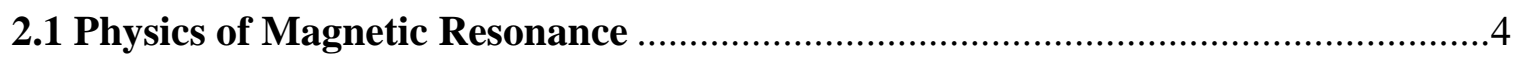

2.1.1 Energy levels, Populations and signal to noise.............................................. 4

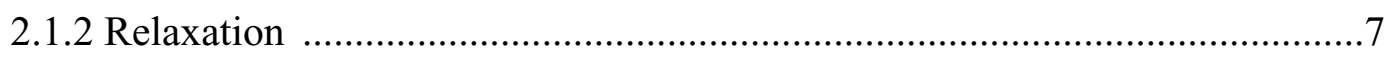

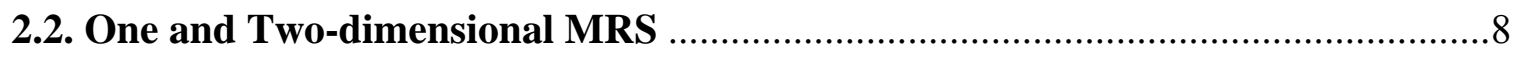

2.2.1. One -Dimensional MRS ........................................................................ 8

2.2.2. Chemical Shifts ............................................................................. 9

2.2.3. Spin-Spin Coupling ......................................................................... 10

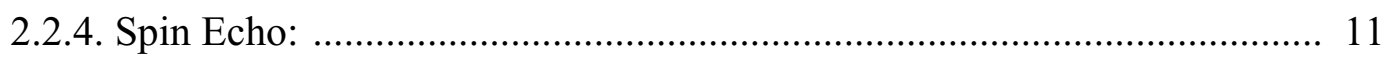

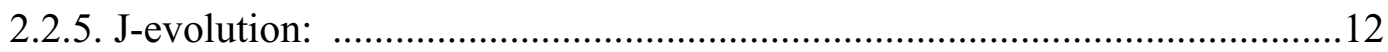

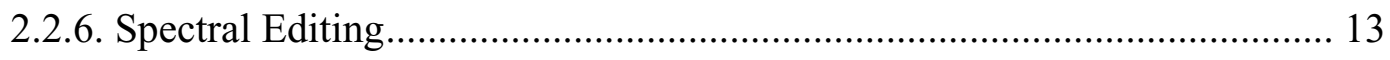

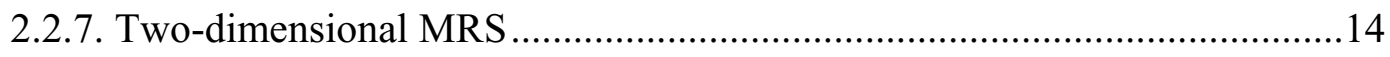

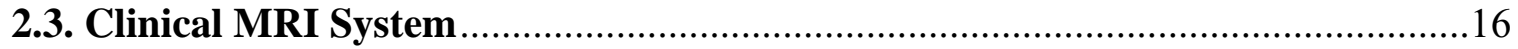

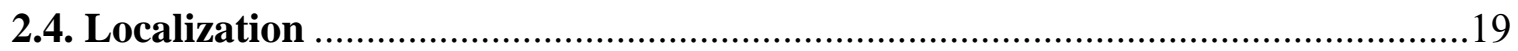

2.4.1. Magnetic Field Gradients....................................................................2

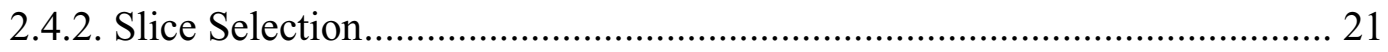

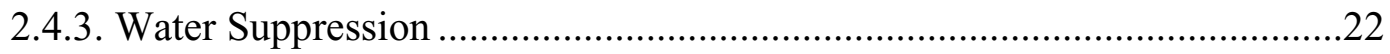

2.4.4. Outer Volume Saturation (OVS) .............................................................23

2.5. Volume Selective Excitation............................................................................................25

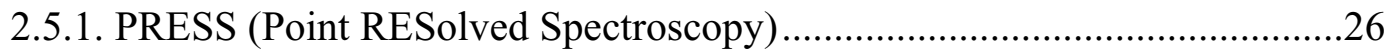

2.5.2. STEAM (Stimulated Echo Acquisition Mode)..............................................27

2.5.3 Limitations of volume localized 1D MRS ……………………………….....28

2.5.4. Localized COrrelated SpectroscopY (LCOSY) ……………………….......28

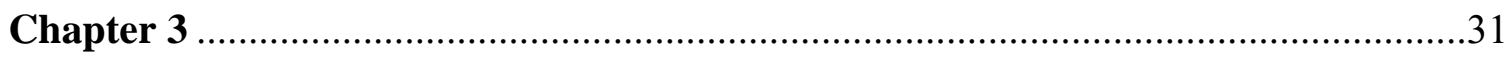

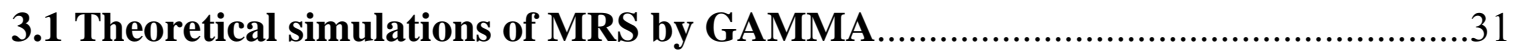

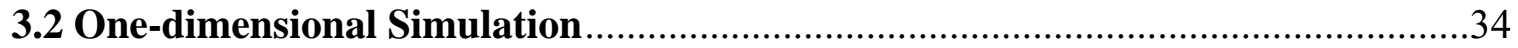

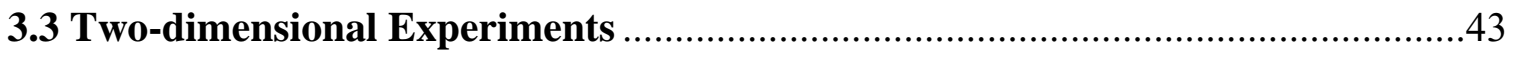


Chapter 4

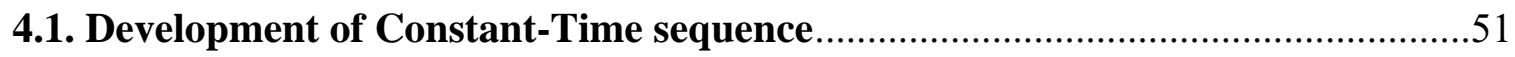

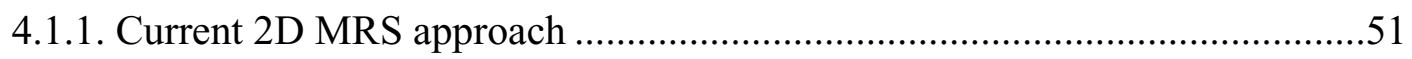

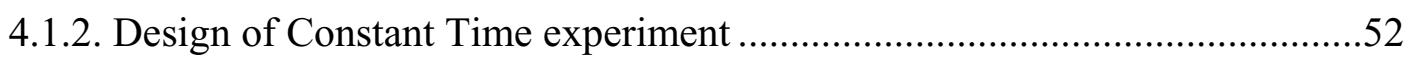

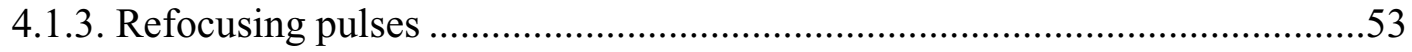

4.2. Implementation of sequence for a GE 3T MRI/MRS scanner .........................55

4.3. Environment for Pulse Programming in C (EPIC) .........................................57

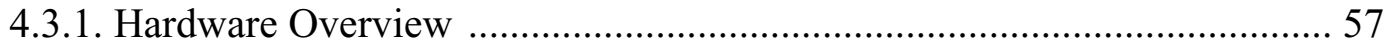

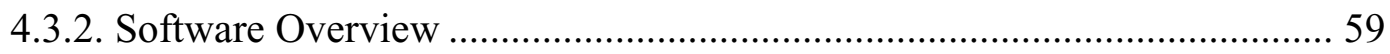

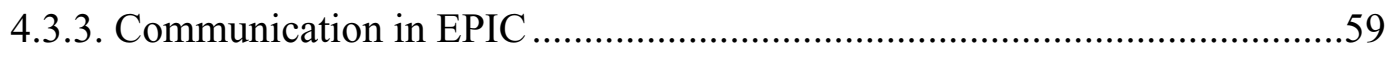

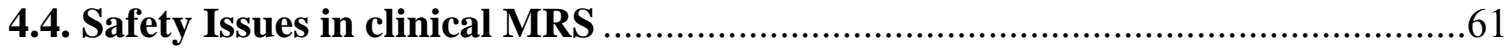

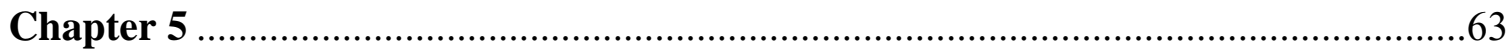

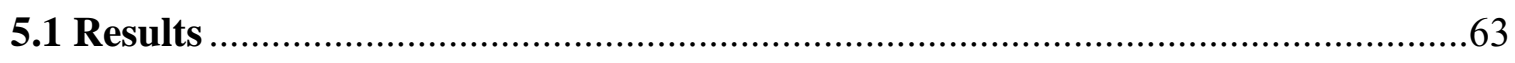

5.1.1 Phantom Preparation ........................................................................63

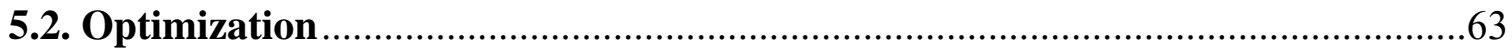

5.2.1. Optimization of solvent suppression............................63

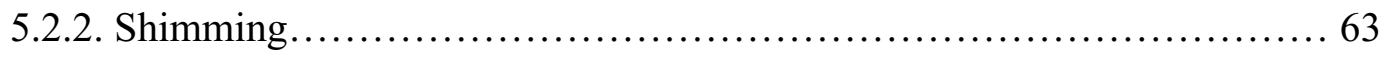

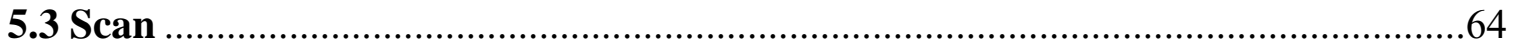

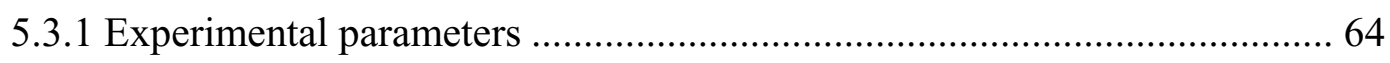

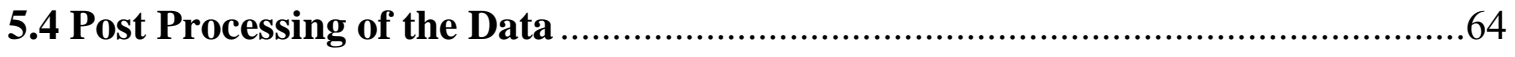

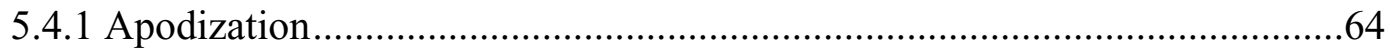

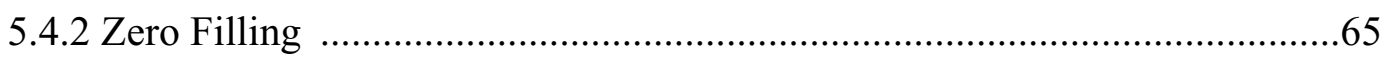

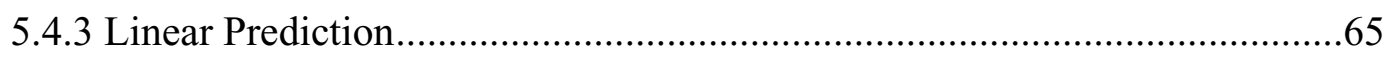

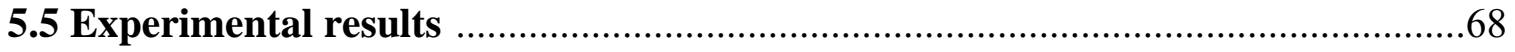

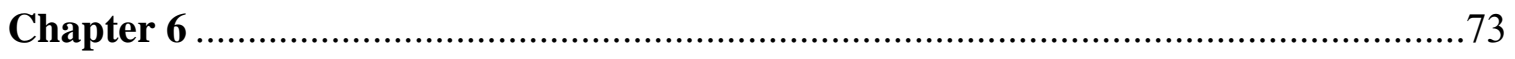

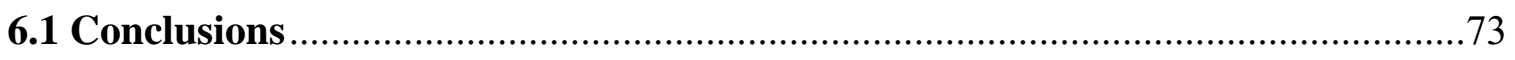

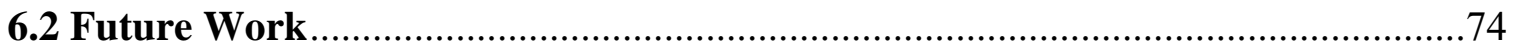

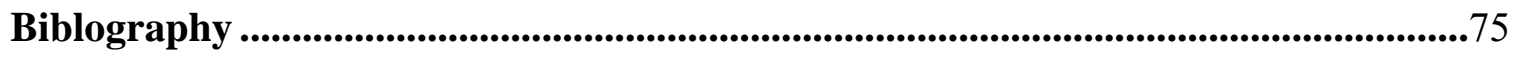




\section{Table of Figures}

FIG. 2.1 Energy level splitting and Boltzmann distribution...............................................

FIG. 2.2 1D spectrum of AB spin system at 3T field strength ..........................................

FIG. 2.3(A) Energy level diagram for a single (uncoupled) nucleus...................................10

FIG. 2.3(B) Energy level diagram for a coupled two-spin system ....................................11

FIG. 2.4 Spin-Echo formation for uncoupled spins ........................................................12

FIG. 2.5 J-Evolution as a function of the echo time in spin echo experiment...................13

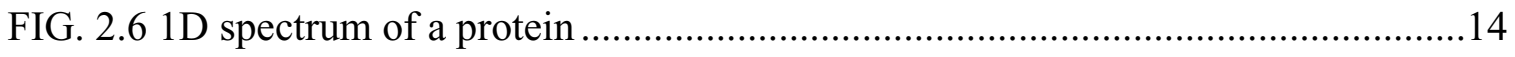

FIG. 2.7 General scheme for two-dimensional MR....................................................14

FIG. 2.8 COSY spectrum for two coupled spins, A and X............................................15

FIG. 2.9 RF system with single RF coil for both transmission and reception....................17

FIG. 2.10 RF system with separate transmitter and receiver coils .....................................18

FIG. 2.11 Brain image showing the selected volume …………………………...........19

FIG. 2.12 Magnetic field distribution by magnetic field gradients in $\mathrm{x}, \mathrm{y}, \mathrm{z}$ directions ....20

FIG. 2.13 Principle of slice selection with magnetic field gradients .................................21

FIG. 2.14 Diagram for water suppression pulse …………………………………….......23

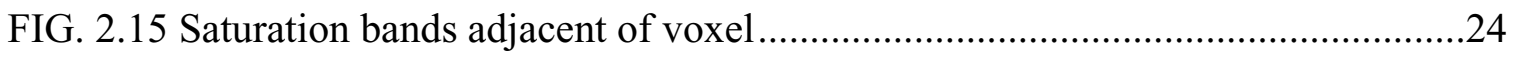

FIG. 2.16 The spectroscopy SAT waveforms...............................................................25

FIG. 2.17 PRESS pulse sequence for 3D spatial localization...........................................26

FIG. 2.18 Echo formation during a sequence containing three RF pulses ........................27

FIG. 2.19 1D MR spectrum of a volume selected from brain ...........................................28

FIG. 2.20 Localized two-dimensional shift correlated MR spectroscopic sequence

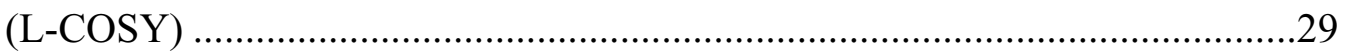

FIG. 2.21 2D L-COSY spectrum of a brain phantom...................................................

FIG. 3.0.0 Theoretical simulation of Acetate at 1.5T ………………...............................35

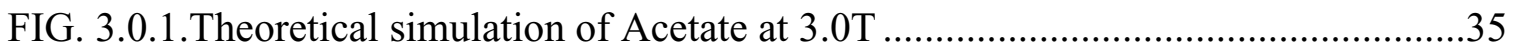

FIG. 3.0.3 Theoretical simulation of Aspartate at 1.5T .....................................................36

FIG. 3.0.4. Theoretical simulation of Aspartate at 3.0T ..................................................36

FIG. 3.0.5 Theoretical simulation of Creatine at 1.5T ....................................................

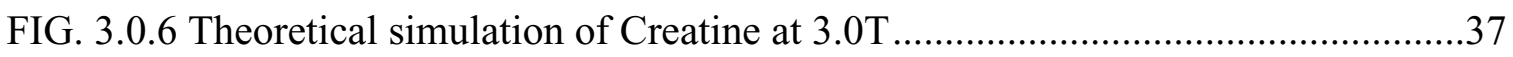

FIG. 3.0.7 Theoretical simulation of GABA at 1.5T .........................................................38 


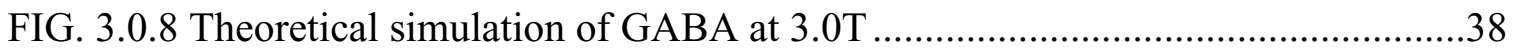

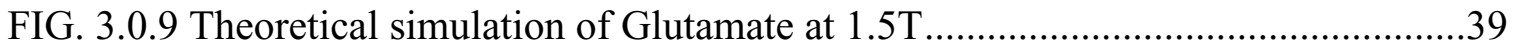

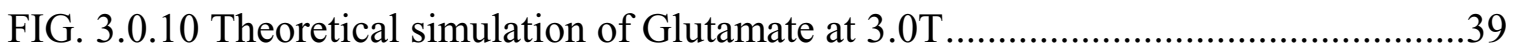

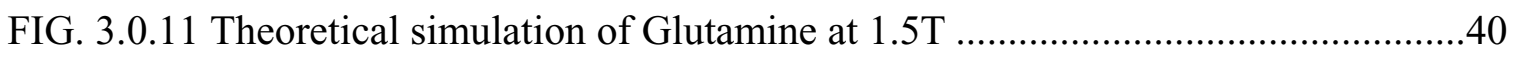

FIG. 3.0.12 Theoretical simulation of Glutamine at 3.0T ..........................................40

FIG. 3.0.13 Theoretical simulation of Lactate at 1.5T .............................................. 41

FIG. 3.0.14 Theoretical simulation of Lactate at 3.0T ................................................41

FIG. 3.0.15 Theoretical simulation of Mio-Inositol at 1.5T .......................................42

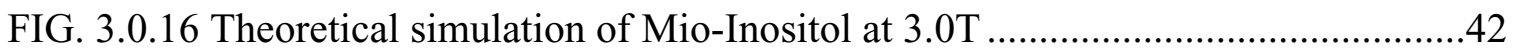

FIG. 3.0.17 Theoretical simulation of Phospho-Creatine at 1.5T ..................................43

FIG. 3.0.18 Theoretical simulation of Phospho-Creatine at 3.0T ..................................43

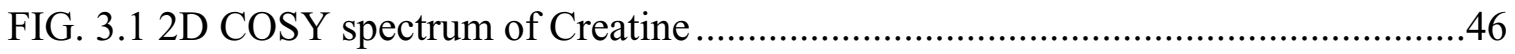

FIG. 3.2 2D COSY spectrum of GABA …......................................................... 47

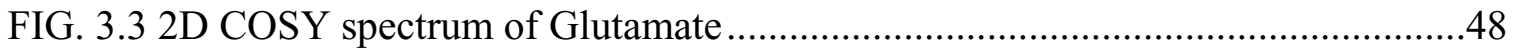

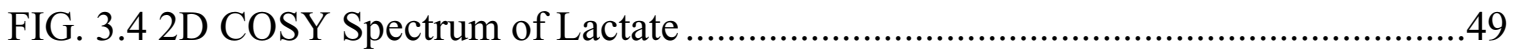

FIG. 3.5 2D COSY spectrum of Myo-Inositol ….....................................................50

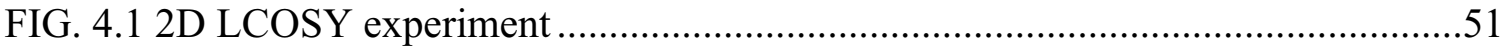

FIG. 4.2 2D Localized Constant-Time Correlated Pulse Sequence.................................56

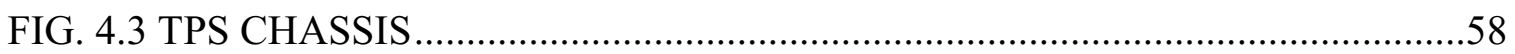

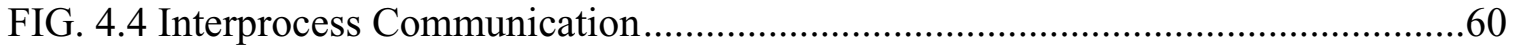

FIG. 5.1 Chemical structure of trans-cinnamic acid ..............................................63

FIG. 5.2 2D L-COSY MR Spectrum of trans-cinnamic acid ......................................66

FIG. 5.3 Simulated 2D COSY MR Spectrum of trans-cinnamic acid ............................67

FIG. 5.4 2D CT-COSY Spectrum $\left(\mathrm{T}_{\mathrm{ct}}=3 / 2 \mathrm{~J}(93 \mathrm{~ms})\right)$............................................69

FIG. 5.5 Simulated spectrum of CT-COSY sequence ............................................ 70

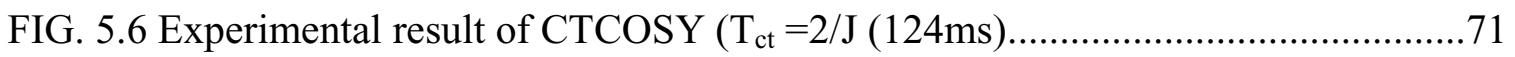

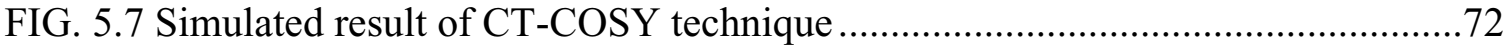




\section{Chapter 1}

\section{Introduction:}

Magnetic Resonance spectroscopy (MRS) is one of the most powerful techniques used for molecular characterization. The predominant developments of universal procedures of coherent spectroscopy within NMR over the past 50 years have found widespread application in a variety of other fields. Many scientists have been honored by Noble Prizes for their key experiments in magnetic resonance starting with Isidor I.Rabi in 1944 for his earliest work. Later Bloch and Purcell shared the 1952 Noble Prize in Physics for the first experimental verifications of the magnetic resonance phenomenon. Prof. R.R. Ernst received the 1991 Nobel Prize in Chemistry, for the methodology development where he introduced a variety of multi-dimensional NMR techniques. Prof. Kurt Wüthrich received the Nobel Prize in Chemistry in 2001 for solving the threedimensional structure of protiens using Magnetic Resonance. In 2003 Prof. Lauterbur and Prof. Mansfield received the Nobel Prize in Medicine for inventing Magnetic Resonance Imaging.

Magnetic Resonance Spectroscopy (MRS) is the study of molecular structure through measurement of the interaction of radio-frequency electromagnetic radiation with a collection of nuclei immersed in a strong magnetic field. A Magnetic Resonance (MR) spectrum can provide detailed information about molecular structure and dynamics, information that would be difficult to obtain from any other method. The physical foundation of MRS lies in the magnetic properties of atomic nuclei. The interaction of the nuclear magnetic moment of the nucleus with an external magnetic field gives rise to splitting of energy levels. This is characterized by absorption and emission of photons by the atoms. The absorption of energy can be detected, amplified and recorded as spectral line, the resonance signal.

Magnetic Resonance Spectroscopy (MRS) has made a quantum leap in the last few decades, becoming a staple tool in such divergent fields as chemistry, physics, materials sciences, biology, and medicine. Over the past 50 years MR spectroscopy has developed into one of the most important techniques for determining the structure and dynamics of molecules. The development of in vivo Magnetic Resonance (MR) has perhaps been even more impressive. Magnetic Resonance Spectroscopy (MRS) has been 
applied in vivo from the early beginnings of MR, although dedicated techniques for in vivo MRS were not developed until the 1970's. In vivo MRS has provided a wealth of non-invasive, spectroscopic information on, for example, energy metabolism, amino acids, and intracellular $\mathrm{pH}$. It has been used both as a fundamental research technique as well as a diagnostic tool in medical research. MRS has not gained the same importance as Magnetic Resonance Imaging (MRI) due to the technical aspects associated with it such as low sensitivity, spatial localization, water suppression and spectral overlap, giving MRS in general a low temporal and spatial resolution. However, its achievements in fundamental research show that in vivo MRS can be an important tool in diagnostic medical research providing unique information about the metabolic status of tissues. In vivo MRS has evolved from relatively simple one or two RF pulse sequences to complex MRS and time-varying magnetic field gradients.

MRS is feasible on any nucleus possessing a magnetic moment. For in vivo MRS applications, the metabolically most interesting nuclei that have this property are proton $\left({ }^{1} \mathrm{H}\right)$, carbon-13 $\left({ }^{13} \mathrm{C}\right)$, phosphorus $\left({ }^{31} \mathrm{P}\right)$, and sodium $\left({ }^{23} \mathrm{Na}\right) .{ }^{1} \mathrm{H}$ MRS allows the detection of a number of important amino acids, and the end product of (anaerobic) glycolysis, lactate. The water signal is a problem for ${ }^{1} \mathrm{H}$ MRS, since the resonances obscure the much smaller resonances arising from the ${ }^{1} \mathrm{H}$-containing metabolites which are present in the millimolar range. Due to the recent developments in MR techniques good quality water suppression is achieved for ${ }^{1} \mathrm{H}$ MRS. Furthermore, the potential of in vivo ${ }^{1} \mathrm{H}$ MRS has not been fully exploited due to the small chemical shift range in which the metabolite signals resonate. Since the ${ }^{1} \mathrm{H}$ chemical shift range is only $\sim 10 \mathrm{ppm}$, many of the numerous metabolite resonances are overlapping, thereby obscuring their presence. Due to severe overlap of the metabolites, an unambiguous assignment of coupled metabolite multiplets is severely hindered at low field strengths (1.5T, 3T).

Many research groups have been working on developing new techniques to resolve the overlapping metabolites for in vivo clinical applications using the approaches of one-dimensional spectral editing, multiple-quantum coherences and two-dimensional MRS. Many localized pulse sequences have been developed. Two-dimensional MR techniques such as Localized Correlated Spectroscopy (L-COSY) have been suggested to 
detect all the overlapping metabolites. These techniques give a complex spectrum, and also pose challenges for absolute quantification of the metabolites.

In this thesis we have developed a novel localized two-dimensional Constant-Time Correlated pulse sequence (CT-COSY) on a GE 3T MRI/MRS scanner. The sequence is designed to achieve maximum coherence transfer to the desired fragment of the spin cluster.

Further, we have simulated theoretical MR spectra using GAMMA, a C++ library written for simulation of Magnetic Resonance experiments [42]. The MR spectrum of various metabolites is simulated to understand the spectral patterns at different field strengths (1.5T and 3T). The CT-COSY pulse sequence was simulated and compared to the experimental results.

The Thesis is organized as follows:

Chapter 2 introduces the concepts of Magnetic Resonance Spectroscopy. It discusses the magnetic properties of nuclei, chemical shifts, and couplings. It also gives information on the clinical MR system. One and two-dimensional MRS concepts are discussed. Further the current state of the art clinical MRS experiments are described.

Chapter 3 introduces the GAMMA, which is a platform for magnetic resonance techniques written in $\mathrm{C}++$ language. It gives information on how a MR spectrum is obtained and also shows the simulations of various metabolites at different field strengths. The two-dimensional MR spectra of some metabolites are shown.

Chapter 4 describes the design of our new constant-time experiment on a GE 3T MRI/MRS scanner. It also talks about the limitations of the localized correlated experiments. An overview of EPIC (Environment for Pulse Programming in C), a GE software package is given. This chapter also includes the safety issues that are considered in programming the clinical scanners.

Chapter 5 describes the experimental results on our constant-time technique for a twospin $1 / 2$ system. The experimental results on a phantom containing trans-cinnamic acid have been presented. Theoretical simulations are compared with the experimental results.

Chapter 6 presents the conclusions and the future work on our new constant-time localized MRS technique. 


\section{Chapter 2}

\subsection{Physics of Magnetic Resonance:}

Atoms are made up of electrons and nuclei. An atomic nucleus is specified by atomic number, mass number and spin quantum number. The atomic number specifies the number of protons in the nucleus. The mass number specifies the total number of neutrons and protons in the nucleus. Nuclei which possess an odd number of protons, an odd number of neutrons or both exhibit spin phenomena and have a nonzero spin angular momentum $\mathrm{p}(\mathrm{p}=\mathrm{Ih} / 2 \pi)$, where $\mathrm{h}$ is the Planck's constant and I is spin quantum number. Nuclei with odd mass and atomic number possess magnetic moment. In accordance with the principles of quantum mechanics, spin angular momentum can take on the values $1 / 2$, $1,3 / 2,2,5 / 2$, etc. In an external magnetic field nucleus with a spin quantum number I can take up $2 \mathrm{I}+1$ possible eigenstates (spin states, energy levels) related to the magnetic spin quantum number $\mathrm{m}(\mathrm{m}=-\mathrm{I},-\mathrm{I}+1, . \mathrm{I}-1, \mathrm{I})$.

\subsubsection{Energy levels, Populations and Signal to Noise}

When a sample containing nuclear spins $(\mathrm{I}=1 / 2)$ is inserted into a static magnetic field $B_{0}$ the spins in the ensemble of molecules take on either an energy level $m=+1 / 2$ or $m=-1 / 2$. That is the spins precess around the axis of the static magnetic field $B_{0}$ with a $z$ axis projection being parallel ( $\alpha$ spin state) or antiparallel ( $\beta$ spin state) to $B_{0}$. The populations of the $\alpha$ and $\beta$ states are described by the Boltzmann distribution and are almost equal $\left(\sim 10^{-5}\right.$ difference) since the energies involved are fairly small. Nevertheless the small population difference produces an effective magnetization along the $z$-axis. However, there is no magnetization observed in the $x y$-plane since the individual precession frequencies are uncorrelated, e.g. out of phase such that the $x$ or $y$ components average to zero. In order to measure the precession frequencies, the system is disturbed and brought into a non-equilibrium state, which allows monitoring of transverse ( $x$ or $y$ ) magnetization as shown in FIG 2.1. 

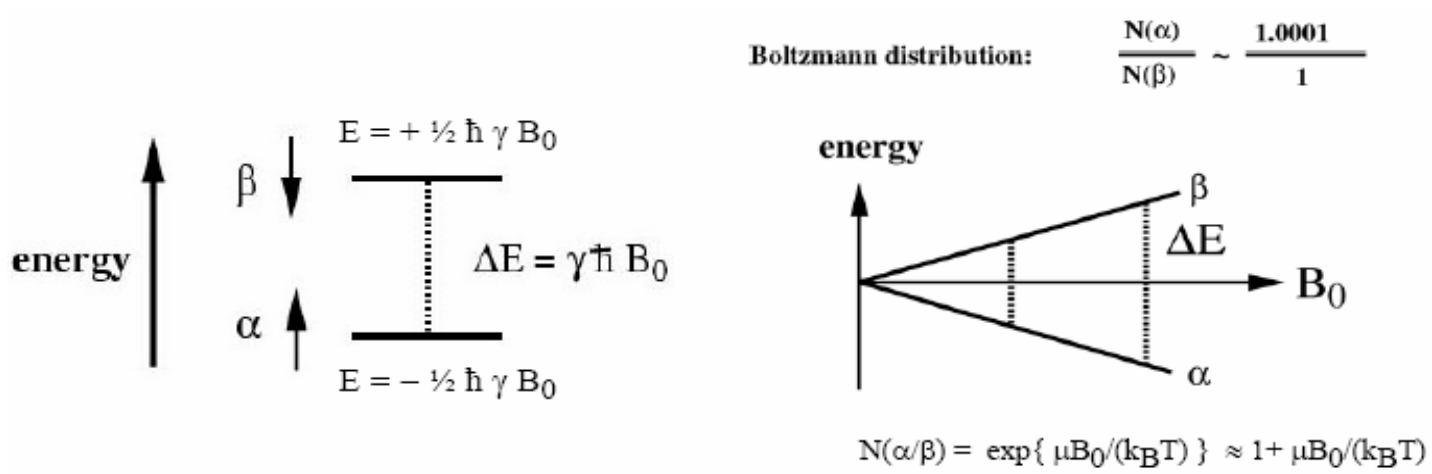

FIG. 2.1 Energy level splitting and Boltzmann distribution [19]

The nuclear magnetic moment precesses in the direction of the field with a frequency known as the Larmor frequency given by

$$
\omega=\gamma B_{0}
$$

where $\gamma$ is the gyromagnetic ratio and $\mathrm{B}_{0}$ is the strength of the magnetic field.

The Larmor frequency depends on the strength of the field $\mathrm{B}_{0}$.

The gyromagnetic ratio $\gamma$, depends on the nucleus and is defined as a constant of proportionality between nuclear angular momentum and magnetic moment.

$$
\gamma=2 \pi \mu / h I
$$

Magnetic Resonance Spectroscopy (MRS) involves resonance frequencies that correspond to radiofrequency wavelengths and is thus located at the low frequency end of the electromagnetic spectrum. The separation of the energy levels defines the population difference $\Delta \mathrm{E}$ (according to Boltzmann statistics) that provides the magnetization and is measured in an MRS experiment. Since $\triangle \mathrm{E}$ is very small MRS is a rather insensitive spectroscopic method, and optimizing the signal-to-noise ratio is always a critical issue for MRS. The signal-to-noise ( $\mathrm{S} / \mathrm{N})$ [47] can be increased by using more spins (higher sample concentration), but also by using higher strengths for the static magnetic field $\left(\mathrm{B}_{0}\right)$, and optimizing the design of MRS experiments, e.g. using spins with the highest $\gamma$ (such as ${ }^{1} \mathrm{H}$ ) for excitation and detection.

$$
\frac{S}{N} \approx n \gamma^{5 / 2} B_{0}^{3 / 2}
$$

Where $\mathrm{n}$ - number of spins (sample concentration), 
$\gamma$ - gyromagnetic ratio,

$\mathrm{B}_{0}$ - static magnetic field strength.

The magnetic moments precess along the z- direction (in the direction of the field $B_{0}$ ) and cancel each other when they are added in transverse plane, perpendicular to field $B_{0}$ and only longitudinal magnetization, parallel to field remains given by

$$
M_{0}=\frac{N \gamma^{2} \hbar^{2} I(I+1)}{3 \kappa T} B_{0}
$$

Where

$\mathrm{N}$ - the number of spins,

$\hbar$ - the Planck's constant divided by $2 \Pi$,

$k$ - the Boltzmann constant,

$T$ - the absolute temperature,

$\gamma$ - gyro magnetic ratio,

I - total magnetic quantum number of the spin.

In order to induce nuclear magnetic resonance, an oscillatory magnetic field has to be applied at the frequency that corresponds to the separation $(\Delta \mathrm{E})$ of the two spin energy levels. In modern Fourier Transform (FT) MR, the $\mathrm{B}_{1}$ (second magnetic field in the transverse plane) is applied as a RF pulse. During the RF pulse, the magnetization will precess about $\mathrm{B}_{0}$ and $\mathrm{B}_{1}$. The initially longitudinal magnetization experiences a torque from the applied $\mathrm{B}_{1}$ field, which results in a rotation of $\mathrm{M}_{0}$ towards the transverse plane. When the $B_{1}$ field is applied for long enough, $M_{0}$ can be completely excited onto the transverse plane or even inverted to the z-axis, giving rise to so-called $90^{\circ}$ and $180^{\circ}$ RF pulses, respectively. The magnetization precesses about $\mathrm{B}_{0}$ at the Larmor frequency and induces an electromotive force (emf) in a receiving coil positioned in the transverse plane. As the nuclear moments precess, they lose phase coherences as a result of interactions among them and magnetic field inhomogeinity effects. Consequently the emf will decrease as a function of time. Thus, the transverse magnetization decreases toward its equilibrium value of zero, and the signal decays. The time-dependence of the emf (or signal intensity) is called the free induction decay (FID).

The response obtained from a FT MRS experiment is a superposition of the frequencies of all spins in the molecule as a function of time, $f(t)$. In order to obtain the 
corresponding spectrum F ( $\omega)$ (intensity as a function of frequency) a Fourier transformation is performed. The Fourier transformation of a time-domain signal $f(t)$ gives a frequency-domain signal $\mathrm{F}(\omega)$ according to

$$
F(\omega)=\int_{-\infty}^{+\infty} f(t) e^{-i \omega t} d t
$$

Fourier transformation is a reversible operation, so that a time-domain signal can be calculated from a frequency-domain signal with an inverse Fourier transformation given by

$$
f(t)=\int_{-\infty}^{+\infty} F(\omega) e^{+i \omega t} d \omega
$$

\subsubsection{Relaxation}

After a $90^{\circ}$ pulse has been applied to the equilibrium $z$-magnetization, transverse magnetization is created in which the phases of the individual spin precession frequencies are correlated. Such a non-random superposition of states is called coherence.

However, the population difference between the $\alpha$ and $\beta$ states (which corresponds to $z$ magnetization) has disappeared. Thus, the system is in a non-equilibrium state and will therefore relax back into the equilibrium state.

The relaxation processes can be explained in two ways: longitudinal (spin-lattice) relaxation and transverse (spin-spin) relaxation.

In longitudinal relaxation, energy is transferred to the molecular frame, the lattice. The half-life for this process is called the spin-lattice relaxation time $\left(\mathrm{T}_{1}\right)$. After the excitation pulse the recovery of magnetization along longitudinal directions is described by $\mathrm{T}_{1}$ which of the order of a few seconds.

In transverse relaxation energy is transferred to neighboring nuclei. The half-life for this process is called the spin-spin relaxation $T_{2}$. After RF pulse is switched off the protons lose phase coherence resulting in dephasing of spins. Thus the transverse magnetization decreases with a decaying with time constant $T_{2}$. The $T_{2}$ is of the order of few tens of milliseconds. The line width of an MRS signal is described by the exponential decay of the FID, and reciprocal to the transverse relaxation time $\mathrm{T}_{2}$. 


\subsection{One and Two-dimensional MRS}

\subsubsection{One-dimensional MRS}

A one-dimensional MRS experiment consists of two sections: preparation and detection. In any pulse sequence, the MRS experiment starts with preparation where the spin system is set to a defined state, magnetization aligned along the main $\mathrm{B}_{0}$ field and ends with detection of a signal that is directly proportional to the magnetization of a selected nucleus in the transverse plane.

The simplest pulse sequence consists of a single $90^{0} \mathrm{RF}$ pulse (applied along the $\mathrm{x}$ axis), which rotates the equilibrium magnetization $\mathrm{M}_{\mathrm{z}}$ onto the $\mathrm{y}$-axis $\left(\mathrm{M}_{\mathrm{y}}\right)$. After this pulse each spin precesses with its own Larmor frequency around the z-axis and induces a signal in the receiver coil. The signal decays due to $\mathrm{T}_{2}$ relaxation and is therefore called free induction decay (FID). Usually, the experiment is repeated several times and the data are summed up to increase the signal to noise ratio. After summation the data are Fourier transformed to yield the final 1D spectrum.

All the information in a one-dimensional spectrum is contained in two dimensions: frequency and intensity. A regular 1D MRS spectrum informs us about the chemical shifts of the nuclei from the positions of the resonances, the sizes of the $J$ coupling constants from the splitting into multiplets, and the relative numbers of spins from the intensities (FIG. 2.2).

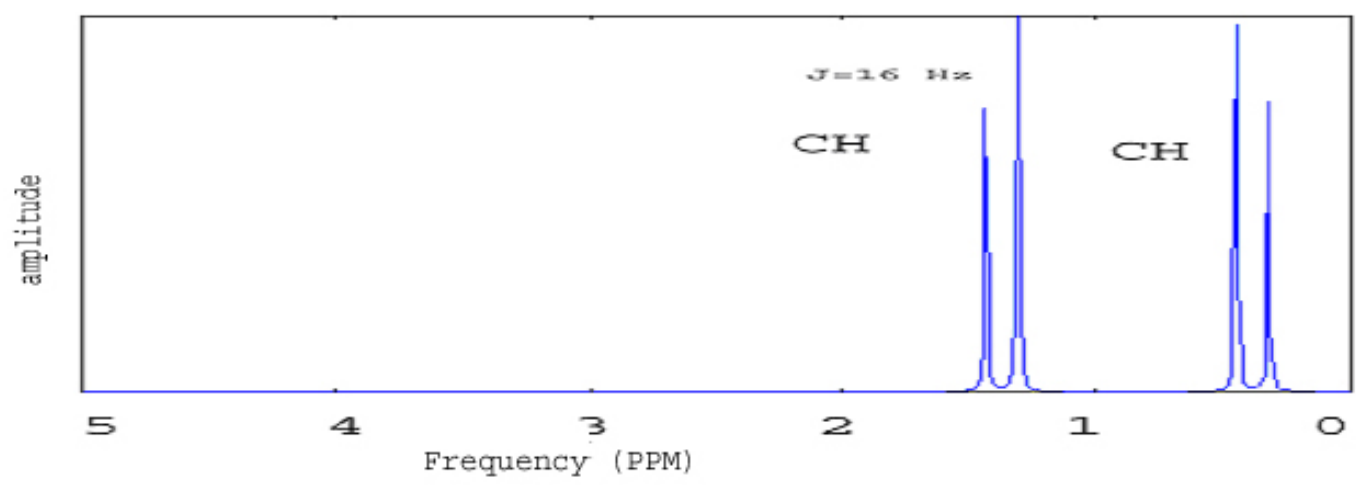

FIG. $2.21 D$ spectrum of AB spin system at $3 T$ field strength 


\subsubsection{Chemical Shifts}

MR spectroscopy in chemistry is significant based on its ability to distinguish a particular nucleus with respect to its environment in the molecule. Chemically different protons in a molecule experience small differences in magnetic field $[16,17]$. The spectrum produced by the different chemical environments of the protons in the molecule, is known as the chemical shift of the resonance frequency.

When an atom is placed in a magnetic field, the nucleus experiences not the magnetic field that is applied to the sample $\left(B_{0}\right)$ but rather the field after it has been altered by the screening or shielding of the electrons surrounding the nucleus. The applied magnetic field $B_{0}$ induces circulations in the electron cloud surrounding the nucleus such that, following Lenz's law, a magnetic moment $\mu$, opposed to $B_{0}$, is produced. Thus the effective magnetic field at the nucleus is less than applied field by a fraction

$$
B_{\text {local }}=B_{0}(1-\sigma)
$$

This effect corresponds to a magnetic shielding of the nucleus that reduces $B_{0}$ by an amount equal to $\sigma B_{0}$.

The resonance frequency varies with field strength and it is technically difficult to obtain an absolute determination of field strength. Hence chemical shifts are measured and reported relative to some agreed reference substance. In proton MRS, the compound used under normal circumstances is TetraMethylSilane (TMS), the twelve protons of which give a sharp signal that is always recorded simultaneously with the spectrum of the sample under investigation.

In an applied magnetic field $B_{o}$ the sample and reference materials, with shielding $\sigma_{S}$ and $\sigma_{R}$, respectively, have resonance frequencies $v_{S}$ and $v_{R}$. Chemical shift is defined by

$$
\delta \equiv \frac{v_{S}-v_{R}}{v_{R}} \times 10^{6}
$$

The $\delta$ scale is clearly dimensionless, but with the factor of $10^{-6}, \delta$ has the units of parts per million (ppm) independent of the RF frequency or magnetic field strength. 
We choose letters of the alphabet representative of relative chemical shifts, say, A, B, X. For nuclei that have a small chemical shift relative to each other, two letters of the alphabet that are close to each other are chosen and for nuclei with large relative chemical shifts, letters from opposite ends of the alphabet are chosen.

\subsubsection{Spin-Spin Coupling}

The important features that characterize MRS spectra are resonant frequencies, signal amplitudes and splitting of resonance lines into several smaller lines. Nonequivalent nuclei (different chemical shifts) that are close to one another exert an influence on each other's effective magnetic field. This leads to splitting of resonance lines into many smaller lines in MRS spectra. We notice not only a difference in the position of the resonance signals but also a difference in the multiplicity of the MRS signals. This interaction is called spin-spin (or J) coupling [18].

This phenomenon can be explained in terms of energy level diagrams. For a single nucleus two levels are observed, with an energy difference of $\Delta E=h v$, giving rise to a single resonance line of frequency $v$. In the case of two-coupled nuclei relative to each other there are four energy levels corresponding to the four possible parallel and antiparallel orientations. The vertical lines in FIG. 2.3(A) and FIG. 2.3(B) represent the allowed transitions between energy levels. Allowed transitions is one where the spin of one nucleus changes from spin up to spin down, or spin down to spin up. FIG. 2.3(A) shows the energy levels for an uncoupled spin system, whereas FIG. 2.3 (B) shows for a coupled spin system. Energy difference between the transition levels is given by $\Delta E$.

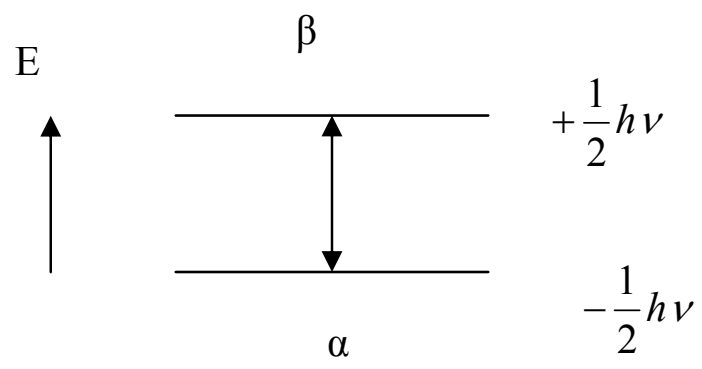

FIG. 2.3(A) Energy level diagram for a single (uncoupled) nucleus 


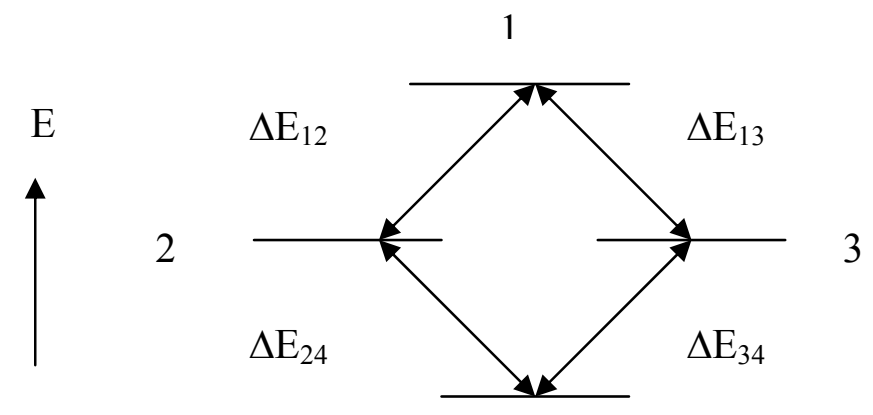

4

FIG. 2.3(B) Energy level diagram for a coupled two-spin system [19]

The magnitude of interaction between nuclei $\mathrm{A}$ and $\mathrm{X}$ is given by a spin-spin coupling constant $J_{A X}$ (often called scalar coupling), which is expressed in frequency units (hertz). The separation between two lines in a spectrum can be identified as a coupling constant, which is independent of the spectrometer frequency. Electrons carry the spin coupling information through chemical bonds. The magnitude of the coupling decreases as the number of the intervening bonds increases.

\subsubsection{Spin Echo:}

E.L. Hahn in 1950 [20] introduced the concept of spin echoes in magnetic resonance. The sequence used two $90^{\circ}$ pulses to show the formation of the echo and was named by Hahn. The simplest experiment to generate spin-echoes is the sequence of two RF pulses shown in FIG. 2.4. Spin echo permits measurement of $T_{2}$ without magnetic field interference. An initial $90^{\circ} \mathrm{RF}$ pulse irradiated along the $\mathrm{x}$-axis creates transverse magnetization in xy plane. During the subsequent delay, the magnetization starts losing coherence due to $\mathrm{T}_{2}$ relaxation and inhomogenity in $\mathrm{B}_{0}$. A $180^{\circ} \mathrm{RF}$ pulse is applied after this delay, which causes all the magnetization vectors to rotate about the y-axis by $180^{\circ}$. In the second delay, which is equal to the first delay, the resultant magnetization, which diminishes in magnitude during the dephasing process with associated free induction signal (FID) signal, is restored during the rephasing, and the signal builds up to a maximum (the echo) before decaying as the magnetization again dephases. The time 
between the $90^{\circ}$ pulse and the top of the spin echo is called as echo time (TE). The spin echo thus reverses the dephasing resulting from the inhomogenity in $\mathrm{B}_{0}$.

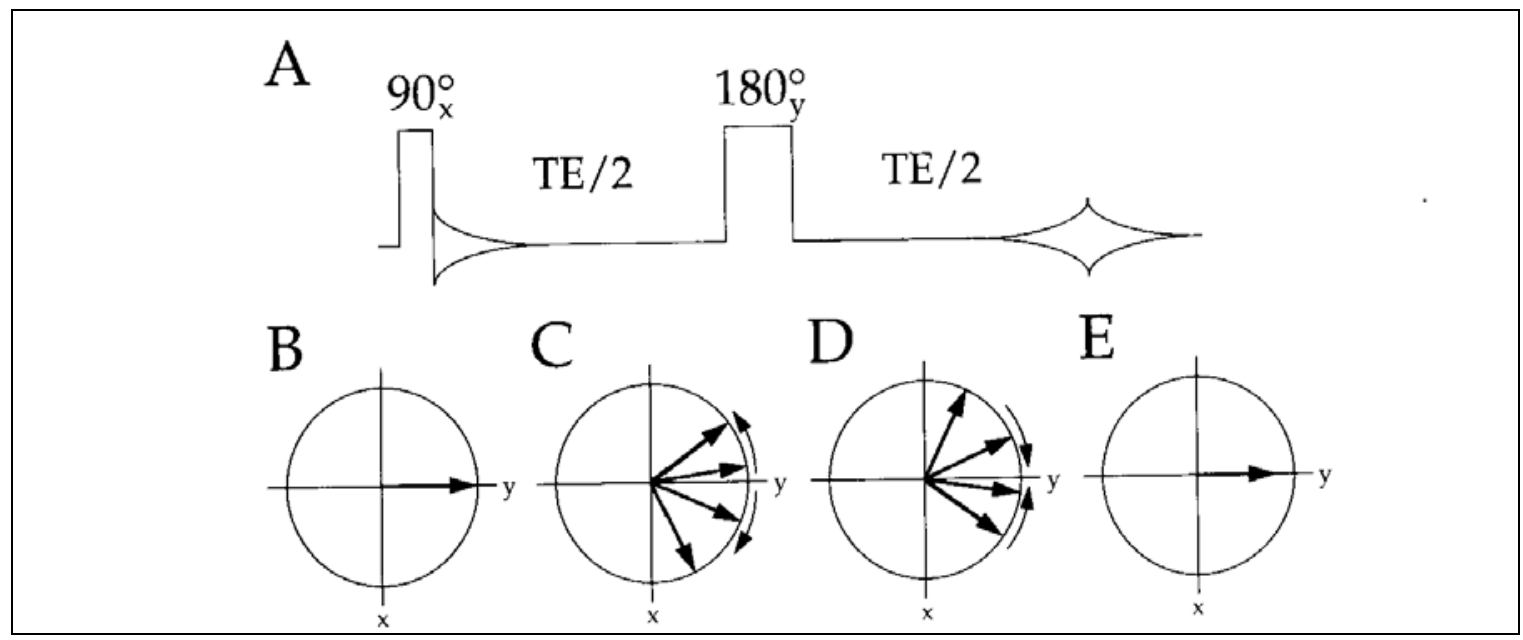

FIG. 2.4 Spin-echo formation for uncoupled spins. In a spin-echo experiment (A) spins are excited, (B) spins dephase in the transverse plane during first half of the echo time (C) A $180^{\circ}$ refocusing pulse mirrors all magnetization vectors along y-axis (D) after which spins rephrase during the second half of the echo time (E) at the echo time TE refocusing is complete and spin echo is formed [19]

\subsubsection{J-evolution:}

The formation of spin echoes in the case of coupled spins is more complicated. Assume an AX spin system with a coupling constant J. The NMR spectrum of an AX spin system consists of two doublets centered around the resonance frequencies of $\mathrm{A}$ and $\mathrm{X}$ respectively. After applying the first $90^{\circ}$ pulse the magnetization is aligned along the $\mathrm{y}$ axis. During the first half of the echo time (TE/2), the magnetization is spreading out due to $\mathrm{B}_{0}$ inhomogenities and frequency offsets. The resonance frequencies of the two doublets are given by $\left(v_{\mathrm{A}}+\mathrm{J} / 2\right),\left(v_{\mathrm{A}}-\mathrm{J} / 2\right),\left(v_{\mathrm{X}}+\mathrm{J} / 2\right),\left(v_{\mathrm{X}}-\mathrm{J} / 2\right)$. The frequency offsets for coupled spins are expressed in terms of $\mathrm{J}$ coupling constant. We can observe that half of the spins are precessing $\mathrm{J} / 2$ Hertz slower and the other half $\mathrm{J} / 2$ Hertz faster, than the center frequencies $v_{\mathrm{A}}, v_{\mathrm{X}}$. The $180^{\circ}$ pulse applied after the delay inverts the spin populations of both resonances, thereby causing an interchange of the transition energies and of the resonance frequencies. The spins with resonance frequency $\left(v_{\mathrm{A}}+\mathrm{J} / 2\right)$ before $180^{\circ}$ pulse will precess with resonance frequency $\left(v_{\mathrm{A}}-\mathrm{J} / 2\right)$ after the $180^{\circ}$ pulse. During a spin-echo, $\mathrm{B}_{0}$ inhomogeneities and frequency offsets are always refocused (both for uncoupled and coupled spins), but for coupled spins the evolution due to $\mathrm{J}$ coupling ( $\mathrm{J}$ 
evolution) is not refocused. The spectra of the coupled spins due to $J$ evolution acquired with a spin-echo experiment are given in FIG. 2.5.

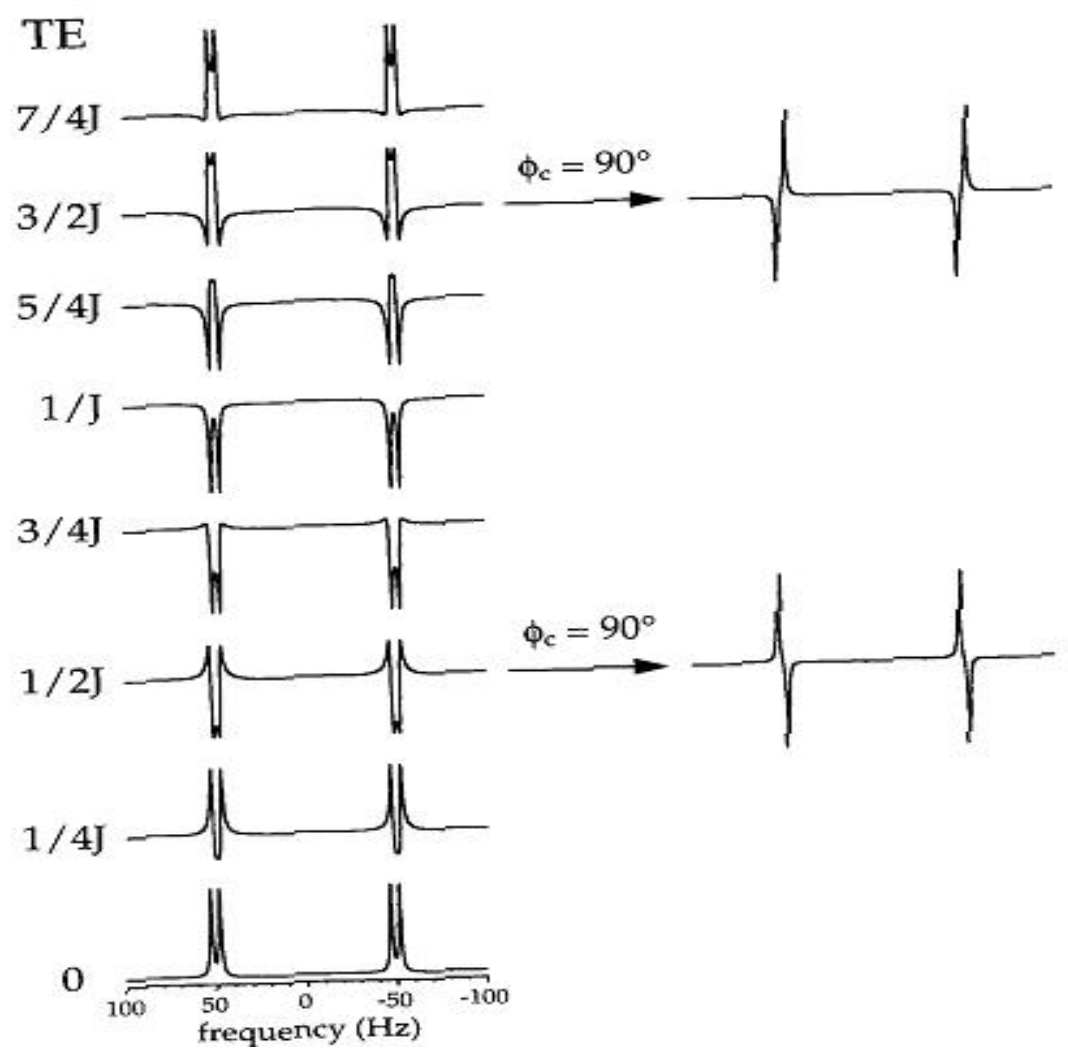

FIG. 2.5 J-evolution as a function of the echo time in spin echo experiment [19]

From FIG. 2.5 it can be seen that for $\mathrm{TE}=0$, the spectrum appears the same as acquired with a $90^{\circ}$-acquisition experiment. For $\mathrm{TE}=1 / \mathrm{J}$ the acquired phase is $\pi$ and the magnetization is said to be in-phase. For $\mathrm{TE}=1 / 2 \mathrm{~J}$ the magnetization is in anti-phase, the positions in the transverse plane of the two resonance lines within one doublet are opposite. J evolution can be used for identification of resonances. For example, in the NMR spectrum with $T E=144 \mathrm{~ms}$, the lactate doublet appears negative with respect to the other (uncoupled) resonances.

\subsubsection{Spectral Editing}

A one-dimensional proton MRS spectrum appears complicated with many overlapping peaks resonating in a small chemical shift range (FIG. 2.5). This complicates unambiguous peak assignment and quantification. Spectral editing may include all techniques, which can simplify a MR spectrum in order to limit the detection to specific 
metabolites. Two-dimensional MRS can be seen as the ultimate spectral editing technique, making no assumptions about the spin system under investigation. FIG. 2.6 shows the 1D spectrum of a protein.

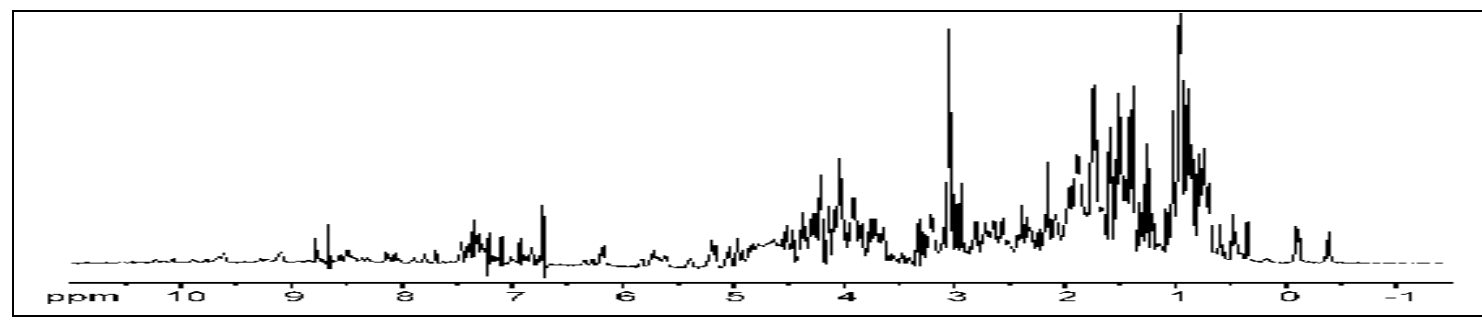

FIG. 2.6 1D spectrum of a protein

Various techniques are used for spectral editing to resolve the overlapping resonances. Spectral editing using Multiple Quantum Coherence is one approach. This approach uses excitation of higher quantum coherences for resolving overlapping resonances. Another approach of spectral editing is to use J-coupling between scalar coupled spins to discriminate coupled from uncoupled spins.

\subsubsection{Two-dimensional MRS}

When the intricacy of the spin system increases the 1D spectrum becomes very complex and the elucidation of the spectrum becomes challenging. From a 1D spectrum it is difficult to determine which spin is coupled to which other spin as they are too complex for interpretation as most of the signals overlap heavily.

Two-dimensional MRS offers relief from overcrowding of resonance lines and the possibility to correlate pairs of resonances. In two-dimensional MRS a signal is recorded as a function of two time variables, $t_{1}$ and $t_{2}$, and the resulting data Fourier transformed twice to yield a spectrum, which is a function of two frequency variables. The intensity is plotted as a function of two frequencies, usually called $F_{1}$ and $F_{2}$ unlike the conventional 1D MRS spectra, which are plots of intensity $v s$. frequency.

General 2D spectroscopy consist of four sections [21] as shown in FIG. 2.7

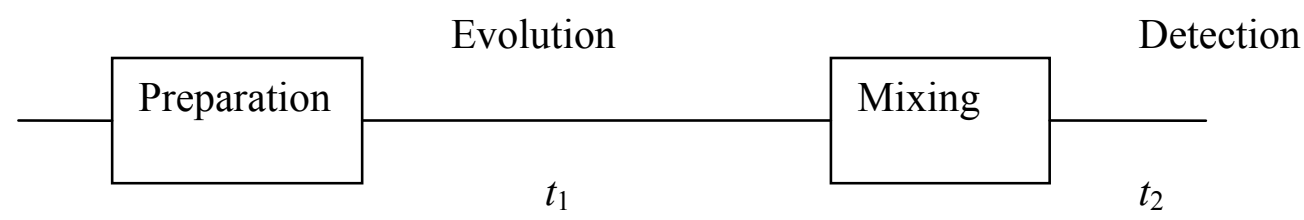

FIG. 2.7 General scheme for two-dimensional MR 
In first period called preparation time, one or more RF pulses excite the spins. The resulting magnetization is allowed to evolve for the first time period $t_{1}$. Further pulses may be applied in mixing time followed by detection time where signal is recorded as a function of the second time variable $t_{2}$.

The recording of the signal can be explained in the following steps. First, $t_{1}$ is set to zero, the pulse sequence is executed and the resulting free induction decay (FID) is recorded. Then the magnetization is allowed to return to equilibrium, then $t_{1}$ is set to $t_{1+} \Delta t_{l}\left(\Delta t_{1}\right.$ is the sampling interval in $\left.t_{1}\right)$, the sequence is repeated and FID is recorded and stored separately from the first. Thus we record a series of spectra, while systematically incrementing the duration $t_{1}$. In the $t_{l}$ dimension the dwell time, $\Delta t_{l}$ is just the fixed duration with which the time $t_{1}$ is incremented from one experiment to the next. Thus, we end up with a discrete matrix $F\left(t_{1}, t_{2}\right)$ of the signal $F$ as a function of $t_{1}$ and $t_{2}$. The $t_{2}$ dimension is represented by the rows of matrix $F$ and the $t_{1}$ dimension by the columns. Fourier transformation of all rows converts them to the frequency domain resulting in a matrix $F\left(t_{1}, F_{2}\right)$. Next all columns are Fourier transformed and the final 2D spectrum $F$ $\left(F_{1}, F_{2}\right)$ is obtained. This matrix is generally represented as a contour plot. In the spectra, scalar coupling giving rise to multiplets come in two distinct types: diagonal-peak multiplets which are centered on the same $F_{1}$ and $F_{2}$ frequency coordinates and crosspeak multiplets which are centered on different $F_{1}$ and $F_{2}$ coordinates.

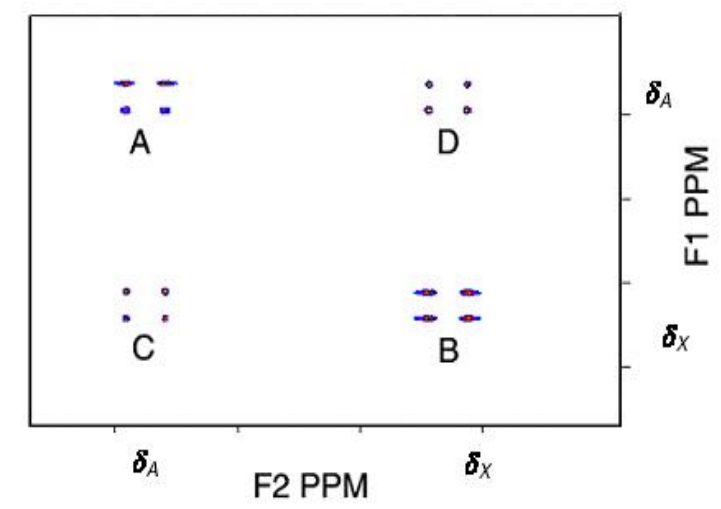

FIG. 2.8 COSY spectrum for two coupled spins, $A$ and $X$ 
Thus in the COSY spectrum (FIG. 2.8) we can observe two diagonal-peak multiplets (A, B) centered at $F 1=F 2=\delta_{\mathrm{A}}$ and $F 1=F 2=\delta_{\mathrm{X}}$, one cross-peak multiplet D centered at $F 1$ $=\delta_{\mathrm{A}}, F 2=\delta_{\mathrm{X}}$ and a second cross-peak multiplet $\mathrm{C}$ centered at $F 1=\delta_{\mathrm{X}}, F 2=\delta_{\mathrm{A}}$. The appearance in a COSY spectrum of a cross-peak multiplet $F 1=\delta_{\mathrm{A}}, F 2=\delta_{\mathrm{X}}$ indicates that the two protons at shifts $\delta_{\mathrm{A}}$ and $\delta_{\mathrm{X}}$ have a scalar coupling between them.

\subsection{Clinical MRI System:}

The MRI system consists of the following components, which operate in relation to each other to produce images of various organs of the patient for diagnosis: Magnet system, Gradient system, RF system. The control and operation of these components is synchronized by a control system in association with the main computer, which gets instructions from the operator through the software of the system.

Magnet: In magnetic resonance, field strengths of up to 20 Tesla are employed. For clinical applications FDA has approved up to 3Tesla field strength. Most clinical MRI magnets are superconducting which employ alloys (like niobium, tin, titanium or zirconium) cooled to very low temperatures. The most common form for a superconducting magnet for MRI is a solenoid in horizontal axis. Cooling is achieved by immersing the superconducting coil in a liquid helium bath, usually surrounded by a dewar vessel of liquid nitrogen. The liquid helium keeps the coil temperature at $4.2 \mathrm{~K}$. They lose all electrical resistance and current can be passed through them without any heating effects. This current in the coil will produce a constant magnetic field. The field strength of a superconducting magnet is inherently stable, and the solenoid also has a shielding effect, minimizing any fluctuating external fields generated in the laboratory.

Gradient Coils: MRI and MRS applications require the presence of coils in the bore of the magnet, which can generate additional fields of specified spatial variation. These are called gradient coils. Typically, the magnetic field variations desired consist of linear variations of the static magnetic field $\mathrm{B}_{0}$ that is commonly taken to define the $\mathrm{z}$ direction of the co-ordinate system used in the coil design. Thus three independently driven coils are required which generate fields with constant $G_{x}=d_{z} / d x, G_{y}=d B_{z} / d y$, and $G_{z}=$ $\mathrm{dB}_{\mathrm{z}} / \mathrm{dz}$ respectively, over usefully large volume of space within the larger magnet supplying $\mathrm{B}_{0}$. This enables the complete MRI repertoire of slice selection and formation 
of images of slices of arbitrary orientation. $G_{x}$ and $G_{y}$ are transverse gradients and $G_{z}$ is the longitudinal gradient in the MRI system.

Radio-Frequency System: The RF system consists of transmitter and receiver parts. The transmitter sends out high-power RF pulses, which are applied to excite the protons from the subject. The amplified pulse is radiated by an RF coil on to the patient body. Protons are excited by this RF pulse and slowly come back to their normal status releasing the absorbed RF energy. The NMR signal is then picked up by the transmit RF coil or by a separate receive RF coil.

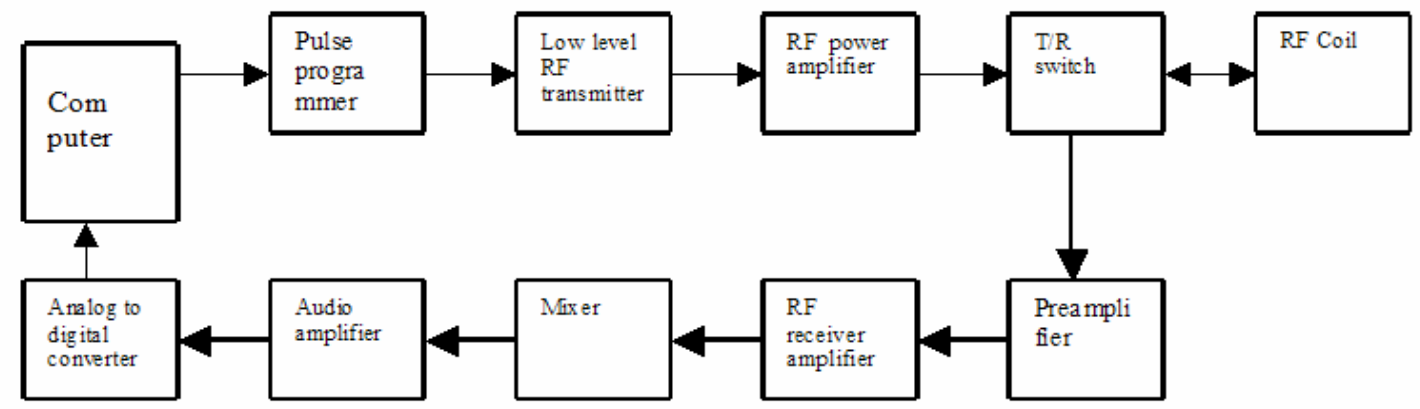

FIG. 2.9 RF system with single RF coil for both transmission and reception

The system computer (or a pulse programmer, which is an attached microprocessor) generates pulse waveforms (FIG. 2.9), which are used to modulate an RF carrier that is an RF signal at constant phase and amplitude. The resulting waveform contains a high frequency signal at the NMR frequency whose amplitude and phase are varied by the modulation. Following the pulse programmer is a low-level RF transmitter followed by an RF power amplifier, which produces watts to kilowatts. The high level RF signal proceeds through the $T / R$ (transmit/receive) switch to the RF coil. The $T / R$ switch is designed to let the high level RF through during transmit but close off the RF transmit channel during the receive. This is because the RF transmit channel may have appreciable noise. The RF signal then produces currents in the RF coil and consequent RF magnetic field, generally called $\mathrm{B}_{1}$ (in contrast to the static magnetic field called as $\mathrm{B}_{0}$ ). The $\mathrm{RF}$ magnetic field in the presence of field gradients manipulates the sample magnetization and nutates particular portions (selected from a slice or volume) away from the direction of $\mathrm{B}_{0}$. Inhomogeneous $\mathrm{B}_{1}$ can cause undesirable non-uniform nutation in different parts of the sample. 
After the magnetization is prepared in the desired way by one or more RF pulses, the transmission is shut off (usually by the transmitter and the T/R switch) and the receive chain gets the NMR signal from the precessing nuclei. The voltage and current induced from the single RF coil by precessing nuclei are first picked up by a preamplifier module. The preamplifier must have a low noise figure, that is, it should add very little noise to the signal coming from the RF coil. The amplified signal from the preamp proceeds through an RF receive amplifier, which further increases the signal strength to a high level. The signal is then fed to a mixer, which 'mixes' the NMR signal with a local RF frequency oscillator. An 'audio' signal is produced whose frequency is the difference between the local oscillator and NMR frequencies.

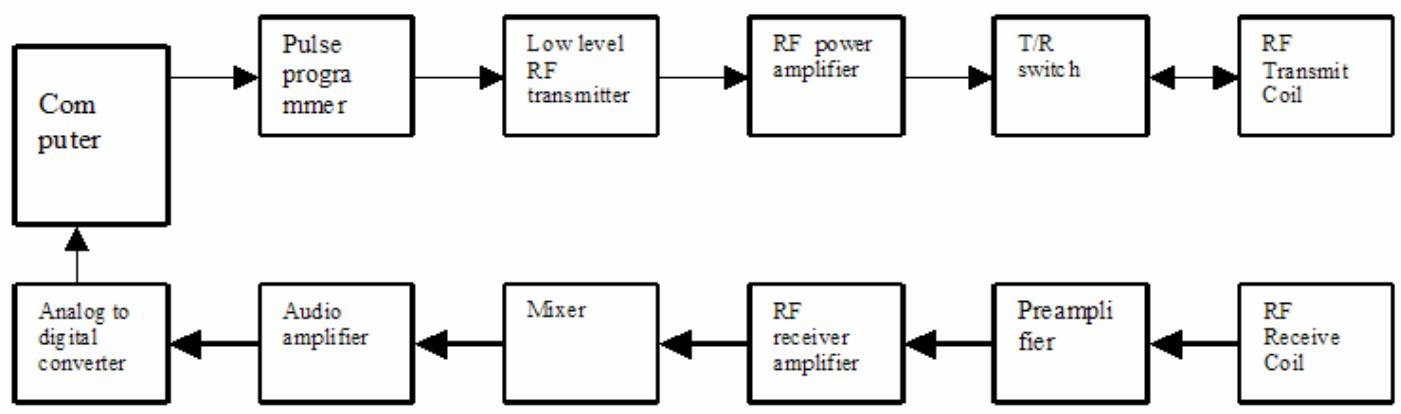

FIG. 2.10 RF system with separate transmitter and receiver coils

The system shown in FIG. 2.10 is similar to that in FIG. 2.9 except that there are separate transmit and receive RF coils. The RF magnetic field $\mathrm{B}_{1}$ which causes the spins to nutate is produced by the transmit coil, and the receive coil senses the returning signal from the precessing nuclear magnetization. Examples of this arrangement might be to transmit with a volume coil and receive with a surface coil. The audio signal is finally digitized (i.e. turned into numbers representing amplitude as a function of time) and fed into the system computer where it is processed to form an image or spectrum.

The transmitter contains a crystal-controlled oscillator at a frequency chosen to match the Larmor frequency of the nuclei under the investigation. This is followed by a power amplifier designed to generate pulses of radio-frequency radiation. The computer controls these pulses because many experiments involve complex sequences of pulses and delays, where duration, intensity and phase of the various pulses are set according to some predetermined strategy for manipulating the nuclear spins. 


\subsection{Localization}

Samples in biological tissues are spatially inhomogeneous in terms of metabolic composition as well as magnetic field strengths $\left(\mathrm{B}_{0}\right.$ and $\left.\mathrm{B}_{1}\right)$. It is essential to employ a spatial localization, for an adequate tissue characterization, such that the origin of the MR signal is accurately known without contamination from other spatial positions. Furthermore, it is difficult to optimize $\mathrm{B}_{0}$ homogeneity over a large unlocalized area, as the broad resonances reduce the spectral resolution and degrade the water suppression. In the case of ${ }^{1} \mathrm{H}$ MRS localization schemes combined with water suppression are required for localized detection of metabolites. FIG. 2.11 shows a brain image where a volume is selected.

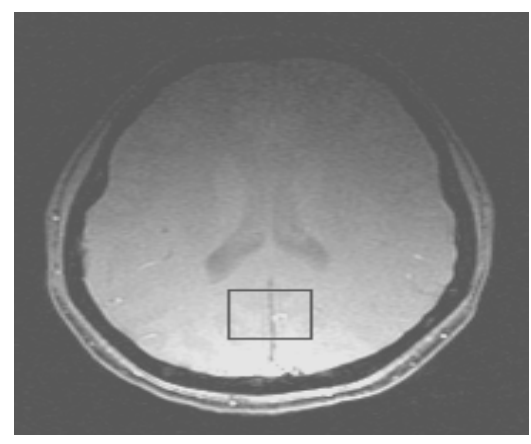

FIG. 2.11 Brain image showing the selected volume [19]

In the last decade, a wide variety of spatial localization techniques have been developed. Spatially dependent gradients, either $\mathrm{B}_{0}$ or $\mathrm{B}_{1}$ gradients, are at the heart of any localization sequence. The $\mathrm{B}_{1}$-dependence of the nutation angle of square $\mathrm{RF}$ pulses (constant amplitude RF pulses) is used to obtain spatial information. The nutation angle $\theta$ generated by a (square) RF pulse of duration $T$ and amplitude $\gamma B_{1}$ is given by

$$
\theta(\mathbf{r})=\gamma \mathrm{B}_{1}(\mathbf{r}) \mathrm{T}
$$

where $\mathbf{r}$ represents the spatial position.

All $\mathrm{B}_{0}$ gradient-based methods depend on the selection of a spatially selective slice by the application of a frequency-selective RF pulse in the presence of a $\mathrm{B}_{0}$ magnetic field.

\subsubsection{Magnetic Field Gradients:}

In MRS instead of providing spectral information, the resonance condition is used to obtain the spatial information. The resonant frequency $\omega_{0}$ is made position dependent, 
such that after Fourier transformation the different frequencies correspond to spatial position rather than chemical shift. This can be achieved by making the external magnetic field position-dependent with the magnetic field gradients [19]. Gradient coils are inserted into the bore of the magnet to superimpose their fields on the main magnetic field. A set of gradient coils provide varying magnetic field strengths along the $x\left(G_{x}\right), y$ $\left(G_{y}\right)$ and $z\left(G_{z}\right)$ direction. It is noted that gradient field does not change the direction of $\mathrm{B}_{0}$ but only changes the spatial variation of the amplitude of $\mathrm{B}_{0}$. The most commonly used magnetic field gradient is a magnetic field of which the amplitude varies linearly with position.

A linear magnetic gradient $G_{z}$ in the z-direction is given by

$$
G_{z}=\left(\frac{d B}{d z}\right)=\text { constant }
$$

where $\mathrm{B}$ is the magnetic field strength and $z$ the position.

The magnetic field is always directed along the z-axis (parallel to main magnetic field $\mathrm{B}_{0}$ ), independent of the gradient orientation. The direction of a gradient refers to the direction in which the strength of the magnetic field varies (Fig 2.12). The magnetic field is centered around the middle of the magnet, i.e. a gradient adds to the main magnetic field on one side of the middle and subtracts from the static field on the other side. Therefore, the magnetic field strength of all gradients is zero in the magnet's isocenter.
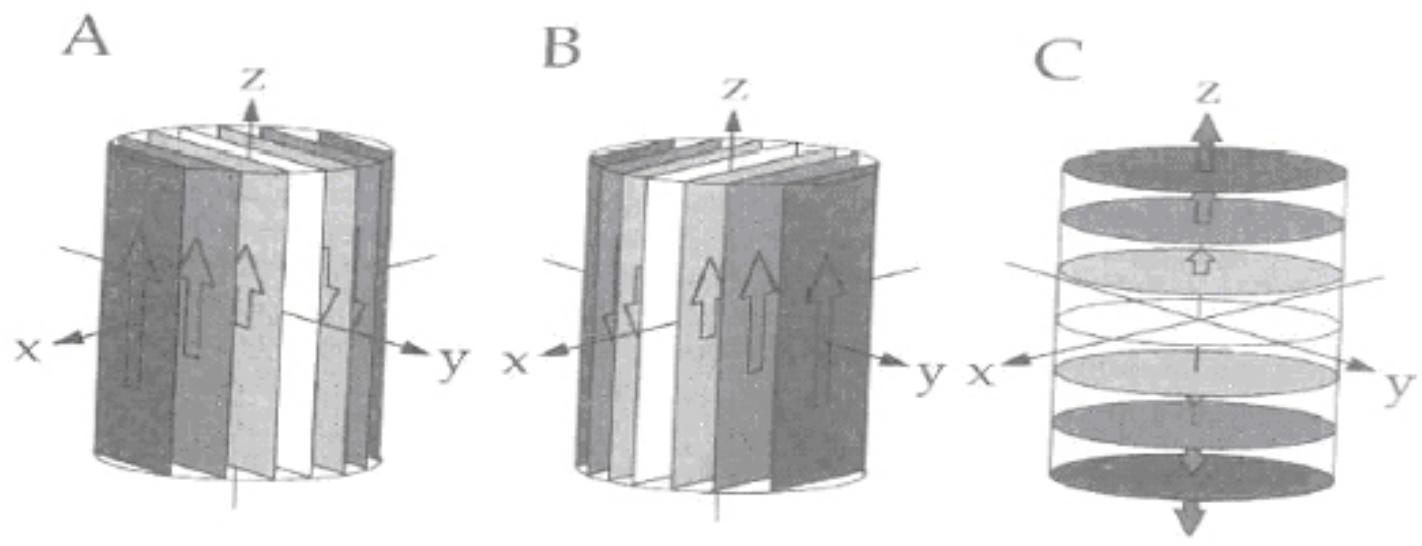

FIG. 2.12 Magnetic field distribution by magnetic field gradients in the (A) X, (B) Y and (C) $Z$ direction. Within each plane the magnetic field strength is identical. The absolute magnetic field generated by the gradient increases linearly as a function of position (as indicated by the relative amplitudes of the arrows and the color of the planes). In the 
middle of the gradient isocenter for each gradient direction (white plane) the magnetic field gradient strength is zero [19]

The total magnetic field at position $\mathbf{r}$ generated by addition of magnetic field gradient $\mathbf{G}$ to the static magnetic field $\mathbf{B}_{\mathbf{0}}$ is given by

$$
\mathbf{B}(\mathbf{r})=\mathbf{B}_{0}+\mathbf{r G}
$$

As a result, the resonance condition should rewritten as

$$
\omega(\mathbf{r})=\gamma \mathbf{B}(\mathbf{r})=\gamma \mathbf{B}_{0}+\gamma \mathbf{r} \mathbf{G}
$$

i.e. the resonant frequency is now dependent on position $\mathbf{r}$.

\subsubsection{Slice Selection}

A spatial slice image can be obtained by the combination of an RF pulse and a magnetic field gradient [23-27]. A magnetic field gradient in the $\mathrm{z}$ direction creates a magnetic field distribution as a function of the $\mathrm{z}$ position. As a result, each $\mathrm{z}$ position is characterized by a specific magnetic field and hence a specific resonant frequency. Short, intense (i.e. 'hard'), constant-amplitude RF pulses ('square' RF pulses) are most often used to achieve uniform excitation of the spins across a given frequency range. When an RF pulse, designed to excite only a selective frequency range, is applied simultaneously with the magnetic field gradient, only a selective range of frequencies, and hence positions, is excited.

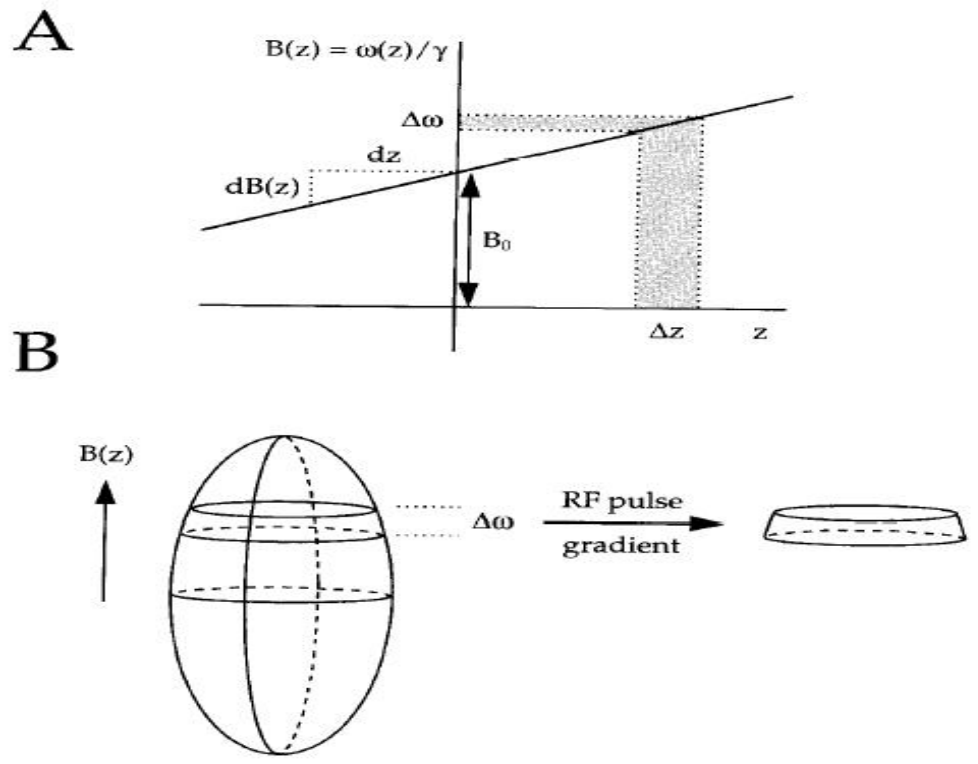

FIG. 2.13 Principle of slice selection with magnetic field gradients [19] 
FIG. 2.13A shows a magnetic field gradient in the $\mathrm{z}$ direction generating a distribution of magnetic field strengths (superimposed on the main magnetic field $\mathrm{B}_{0}$ ) and consequently of frequencies, which is linearly dependent on the spatial position $\mathrm{z}$. Therefore, a range in precession frequencies corresponds directly to a range of spatial positions. FIG 2.13B shows the selection of a range of frequencies by a selective RF pulse in the presence of magnetic field gradient resulting in the selection of a spatial slice from a hypothetical 3D object.

The slice thickness is determined by two factors, the magnetic field gradient strength and the bandwidth of the RF pulse. A stronger magnetic field gradient creates a larger range of resonance frequencies across the sample, resulting in the selection of a thinner spatial slice when the RF pulse is kept constant. Vice versa, the same can be achieved by decreasing the RF pulse bandwidth, while keeping the magnetic field strength constant. When the signal is on resonance, both approaches lead to the same spatial slice.

The spatial position of the slice is also determined by the two factors, the magnetic field gradient strength and the transmitter frequency of the RF pulse. The magnetic field gradient creates the spatial distribution of frequencies, but the transmitter frequency of the RF pulse determines which frequencies are being excited.

\subsubsection{Water Suppression}

In in vivo ${ }^{1} \mathrm{H}$ MRS Studies the water concentration is approximately $10^{5}$ times as abundant as the metabolite concentrations. The concentration of water is 110 Molar as compared to $0.1-2 \mathrm{mM}$ concentration of the metabolites. We have to suppress the strong water signal in order to detect these metabolites. One of the most commonly used pulse sequence elements for water suppression used for in vivo application is shown in FIG. 2.14 . 


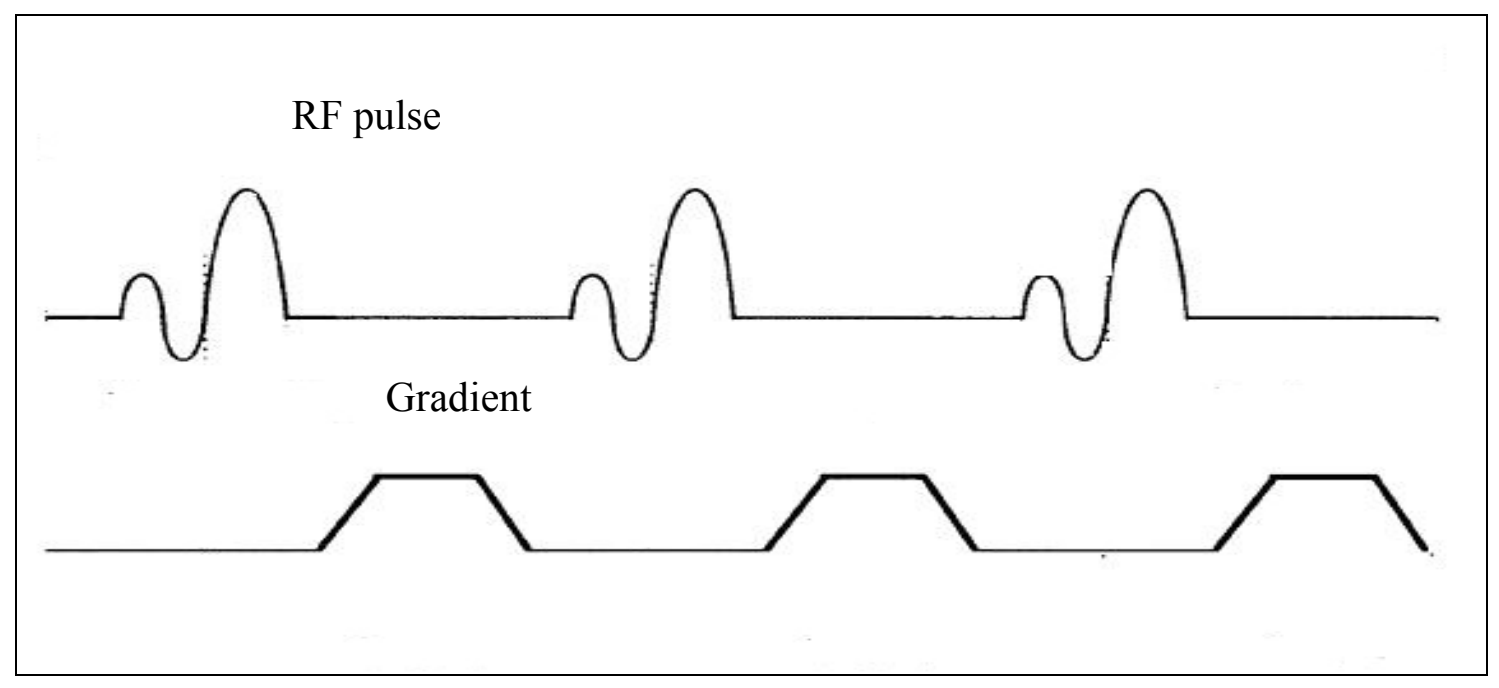

FIG. 2.14 Diagram for water suppression pulse [29]

The selective RF pulse excites the water onto the transverse plane after which all the coherences are dephased by the following $\mathrm{B}_{0}$ magnetic field gradient. This sequence was described by Haase et al [28] and was named as CHESS (Chemical Shift Selective). Since metabolite resonances are left unperturbed, CHESS can precede any pulse sequence. To avoid $T_{1}$ relaxation, the pulse length and the delay between CHESS and the excitation of the metabolites should be as short as possible. The suppression efficiency of the CHESS depends on the ability of the RF pulse to generate transverse magnetization for all water spins in the volume of interest (VOI). Therefore $\mathrm{B}_{0}$ and $\mathrm{B}_{1}$ homogeneity are crucial for adequate water suppression. The CHESS element is repeated two or more times to improve the water suppression.

\subsubsection{Outer Volume Saturation (OVS)}

A three dimensional volume is selected using the combination of RF pulse and $\mathrm{B}_{0}$ gradients. Outer Volume Saturation techniques leave the magnetization in the Volume of Interest (VOI) unperturbed during the localization procedure. All unwanted spins are removed during a preparation period prior to excitation of the desired spins. The dephasing gradients dephase the transverse magnetization in the slice parallel to the coil. This process can be repeated several times to improve the suppression. Up to six spatially localized bands may be saturated during an acquisition to reduce the signal from undesired regions. The spatial saturation RF pulses used in the spectroscopy sequences 
have sharper profiles than the RF pulses used with the standard imaging sequences [29]. Six saturation (SAT) bands are placed adjacent to the prescribed voxel (FIG. 2.15).

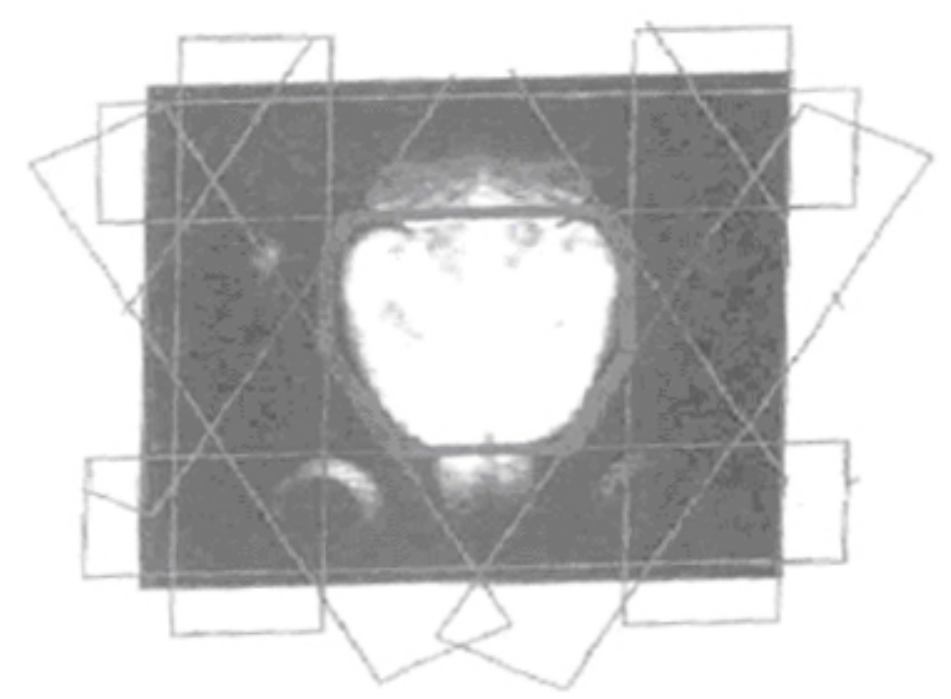

FIG.2.15 Saturation bands adjacent of voxel [19]

Usually the widths of the SAT bands are equal to the corresponding dimensions of the prescribed voxel. The centers of the SAT bands are placed at one-half of the width of the bands from the edges of the voxel. This means that the chemical shift artifact along each axis is similar for both the voxel and for the SAT bands, thereby minimizing possible saturation of portions of the spectrum from within the voxel by the saturation pulses (FIG. 2.16). The effective bandwidth of the SAT pulses is approximately the same as the refocusing pulses, and is thus matched in chemical shift misregistration as prescribed for the volumes defined by the refocusing pulses. 


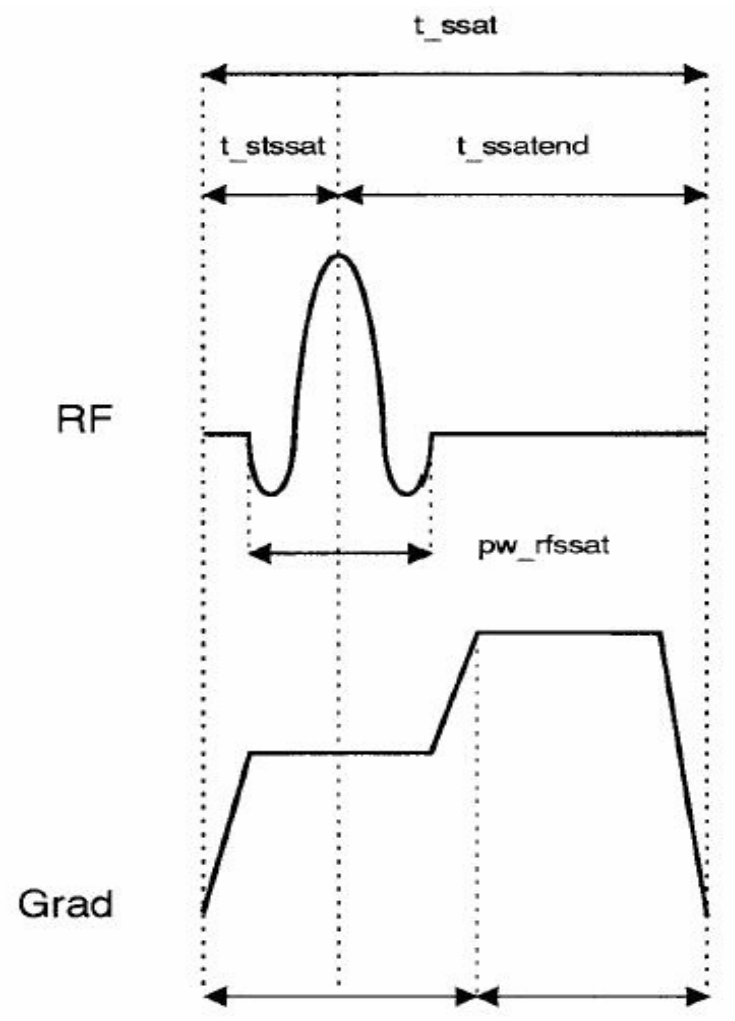

FIG. 2.16 The spectroscopy SAT waveforms [29]

The main advantage of the outer volume suppression techniques for spatial localization is that the magnetization in the Volume of Interest (VOI) remains unperturbed. Further OVS techniques do not induce signal loss due to $T_{1}$ and/or $T_{2}$ relaxation. But for a 3D localization, relatively more RF pulses are required for adequate localization.

\subsection{Volume Selective Excitation}

Single voxel spectroscopy uses three orthogonal slices in such a manner that the region at the intersection of the slices is the only source of signal. Spins within the desired slice experience the pulses while spins outside the desired slice do not experience the selective pulses but are excited onto the transverse plane by the non-selective pulse. We observe the magnetization lies along the longitudinal axis in the three-dimensional cubic voxel experiencing all the RF pulses. 


\subsubsection{PRESS (Point RESolved Spectroscopy)}

The PRESS [30-33] pulse sequence mostly used in clinical applications is shown in FIG. 2.17. PRESS consists of one $90^{\circ} \mathrm{RF}$ pulse and two $180^{\circ} \mathrm{RF}$ pulses. The sequence is capable of three-dimensional localization when the three RF pulses are executed in the presence of $\mathrm{B}_{0}$ magnetic field gradients. A spin echo is formed at time $2 t_{1}$ when the first $180^{\circ}$ pulse is executed at time $t_{1}$ after the excitation pulse. A final spin echo is formed at time $2 t_{1}+2 t_{2}$, when the first spin echo is refocused by the second $180^{\circ}$ pulse during a delay $2 \mathrm{t}_{2}$. The first echo contains the signal from a column, which is the intersection between the two orthogonal slices selected by the $90^{\circ}$ pulse and the first $180^{\circ}$ pulse. The second spin-echo only contains signal from the intersection of the three planes selected by the three pulses resulting in the desired volume. Signal from outside the VOI is either not excited or not refocused, leading to rapid dephasing of the signal. The two pairs of crusher gradients surrounding each $180^{\circ}$ pulse ensure the selection of desired coherences while dephasing all unwanted coherences.

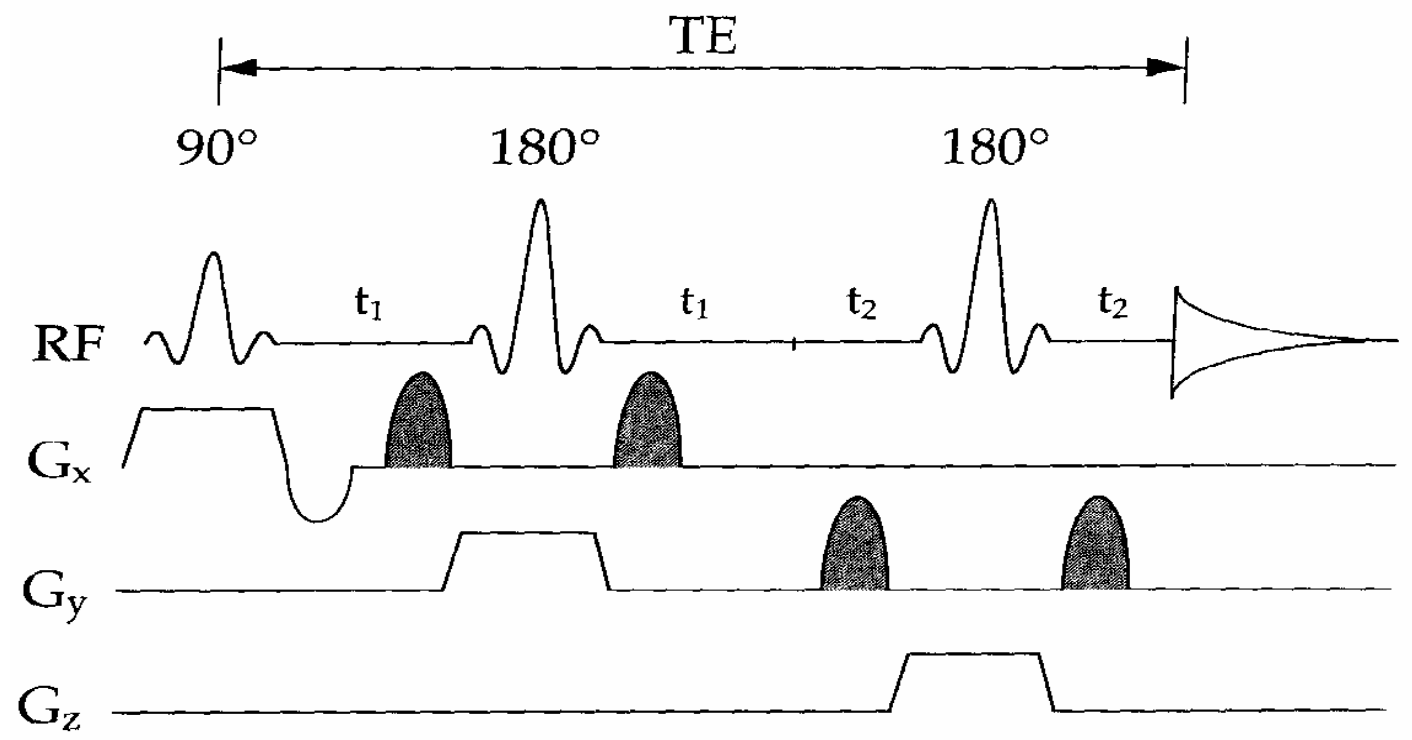

FIG. 2.17 PRESS pulse sequence for 3D spatial localization. Two pairs of crusher gradients surrounding the $180^{\circ}$ refocusing pulses ensure the selection of the desired coherences, while simultaneously destroying all others [19]. 


\subsubsection{STEAM (Stimulated Echo Acquisition Mode)}

STEAM, is a single scan localization technique capable of complete threedimensional localization in a single acquisition. This sequence consists of three $90^{\circ} \mathrm{RF}$ pulses (FIG. 2.18). In this method the volume-of-interest (VOI) is defined by the intersection of three orthogonal slices, which are excited by three frequency selective RF pulses in the presence of orthogonal magnetic field gradients. The first two $90^{\circ}$ pulses are separated by a delay TE/2 and last two $90^{\circ}$ pulses are separated by a delay TM giving rise to four spin echoes. The spin echo formed by pulses 1 and 2 (SE12) occurs at the position TE after the initial excitation pulse, SE13 at position TE+2TM, SE23 at position TE/2+2TM, and SE123 (due to refocusing by pulse 2 and 3 of excited magnetization by pulse 1) occurs at position 2TM. Besides these four spin echoes a stimulated echo formed at position TE+TM is the signal of interest. FID and the spin-echo signals can be eliminated with the use of $\mathrm{B}_{0}$ magnetic field gradients in order to obtain good information from the stimulated echo. Placing a B0 crusher gradient in the TM period can eliminate all the spin-echoes. The FID component can be eliminated by placing a $\mathrm{B}_{0}$ crusher gradient in the first $\mathrm{TE} / 2$ delay and an identical $\mathrm{B}_{0}$ gradient in the last $\mathrm{TE} / 2$ period. Thus a clean selection of the stimulated echo in a single acquisition can be obtained.

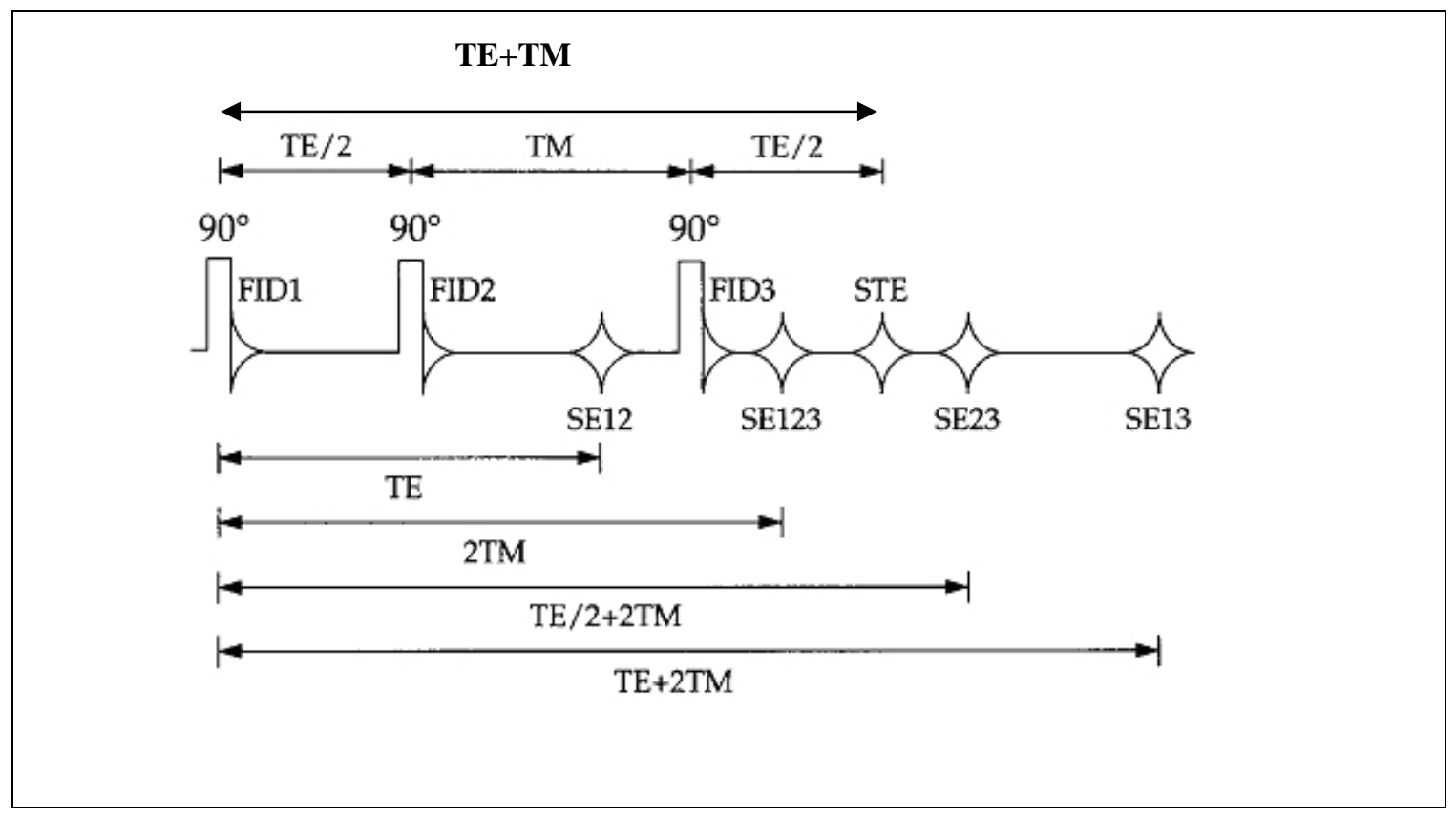

FIG. 2.18 Echo formation during STEAM sequence containing three RF pulses. Three FIDs (FID1, FID2 and FID3), four spin-echoes (SE12, SE13, SE23 and SE123) and one stimulated echo (STE) can be generated [19]. 
The second $90^{\circ} \mathrm{RF}$ pulse rotates half of the transverse magnetization to the longitudinal axis, while the other half is dephased by the TM crusher. This results in $50 \%$ reduction of the amplitude of a stimulated echo.

\subsubsection{Limitations of volume localized 1D MRS:}

FIG. 2.19 shows the 1D spectrum of metabolites in a volume selected from the brain. At lower fields these metabolites overlap extensively. Only a few metabolites are shown in the figure but, in general, the number of metabolites is more. This will further increase the complexity and makes the interpretation of the MR spectrum difficult.

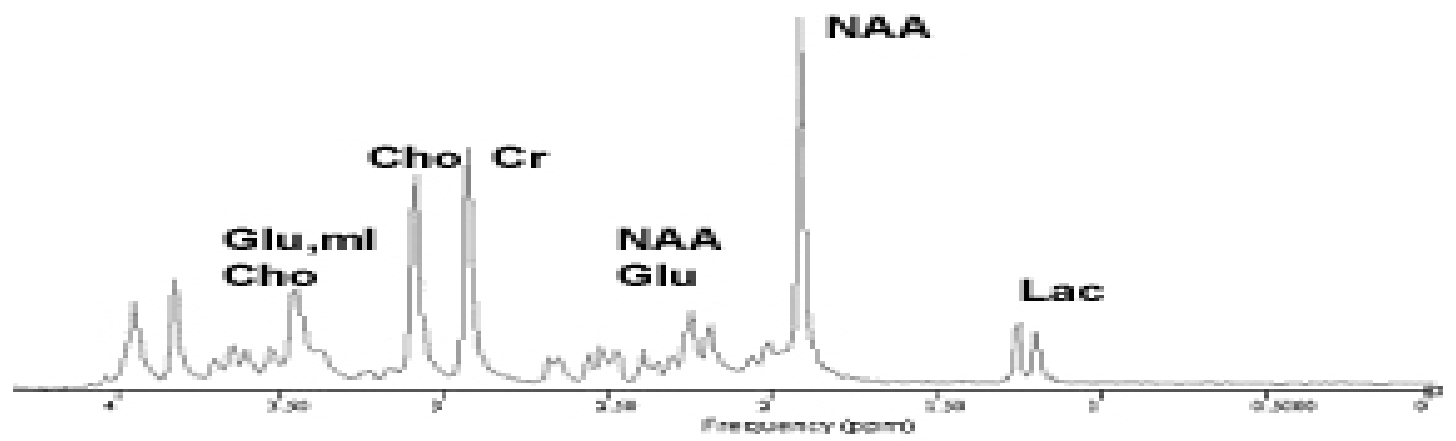

FIG. 2.19 1D MR spectrum of a volume selected from brain.

To avoid such complications many groups have suggested a 2D volume localized MR techniques. Recently a research group from UCLA [41] has proposed a 2D Localized Correlated Spectroscopy (LCOSY) technique.

\subsubsection{Localized COrrelated SpectroscopY (LCOSY):}

LCOSY is a two-dimensional shift correlated MR spectroscopic sequence developed by a group from UCLA [41]. The VOI was localized in one shot by a

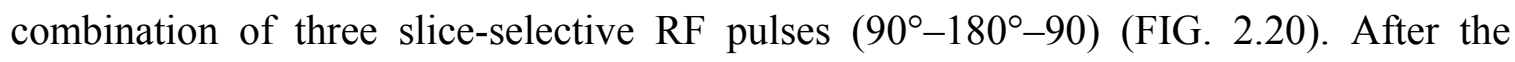
formation of spin echo an incremental period is inserted between $180^{\circ}$ pulse and second $90^{\circ}$ pulse. The last slice selective $90^{\circ} \mathrm{RF}$ pulse acts as coherence transfer pulse for the $2 \mathrm{D}$ spectrum. A pair of refocusing $\mathrm{B}_{0}$ gradient crusher pulses is applied before and after the slice selective $180^{\circ}$ pulse and the last $90^{\circ} \mathrm{RF}$ pulse. For every incremental period a 
minimum of eight averages are obtained to improve signal to noise ratio. The proposed sequence can be considered a single-shot technique in terms of volume localization and coherence transfer.

A CHESS sequence consisting of three frequency-selective water suppression pulses each followed by the dephasing $B_{0}$ gradient pulses is used prior to the localization sequence.

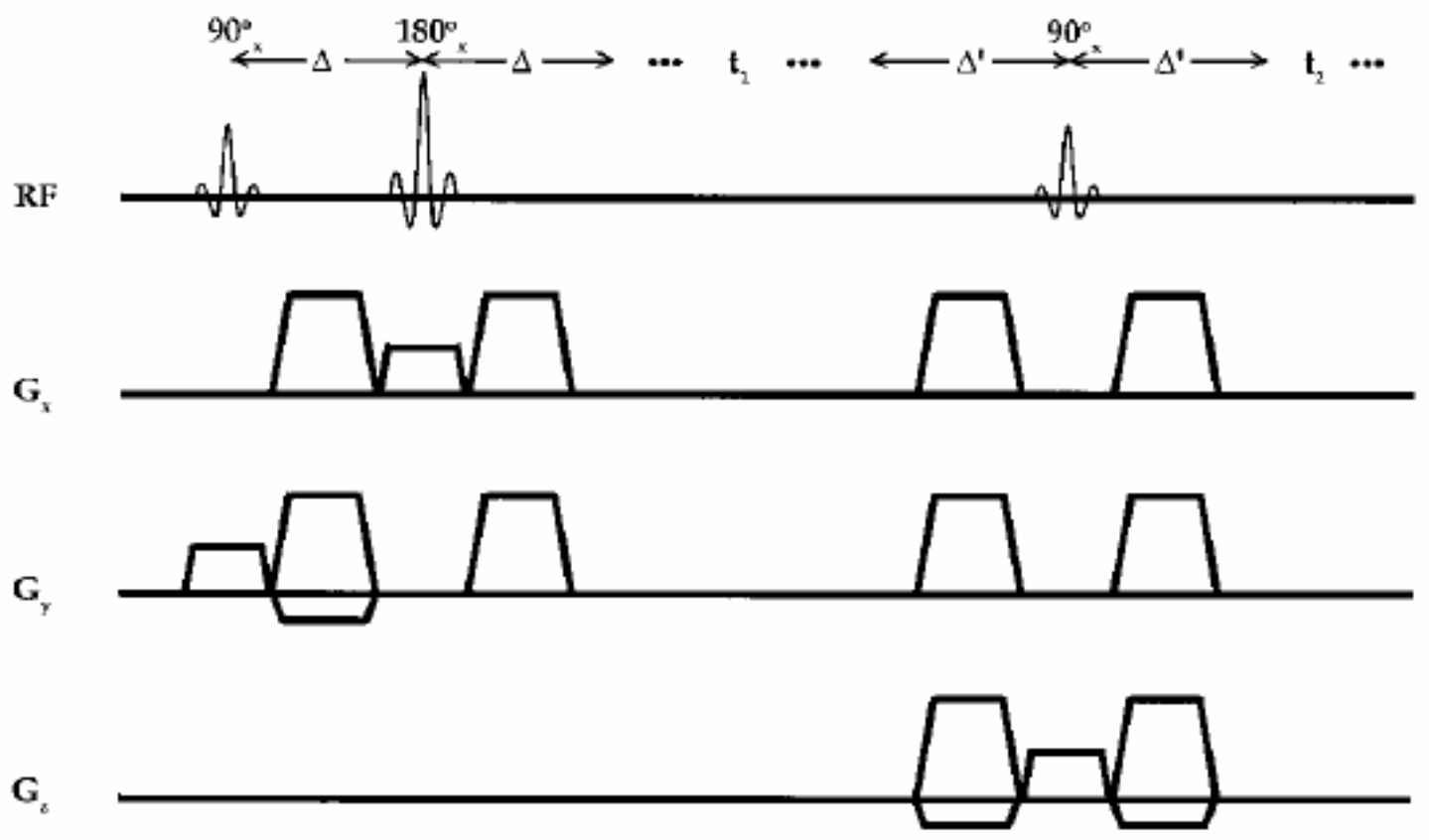

FIG. 2.20 Localized two-dimensional shift correlated MR spectroscopic sequence (LCOSY) [41].

The RF pulse scheme consisted of three RF pulses $\left(90^{\circ}, 180^{\circ}, 90^{\circ}\right)$, which were slice-selective along three orthogonal axes. A pair of $B_{0}$ gradient crusher pulses was symmetric with respect to the slice-refocusing $180^{\circ} \mathrm{RF}$ pulse. The last slice-selective $90^{\circ}$ RF pulse with a pair of symmetric $B_{0}$ gradient crushers also served as coherence transfer pulse for the L-COSY spectrum. FIG. 2.21 shows the 2D spectrum of L-COSY for a volume selected from the brain. We see that the overlapping metabolites which were difficult to characterize in $1 \mathrm{D}$ are now well resolved. The diagonal axis represents the 1D spectrum. Since the protons are coupled to each other we see additional cross peaks. 


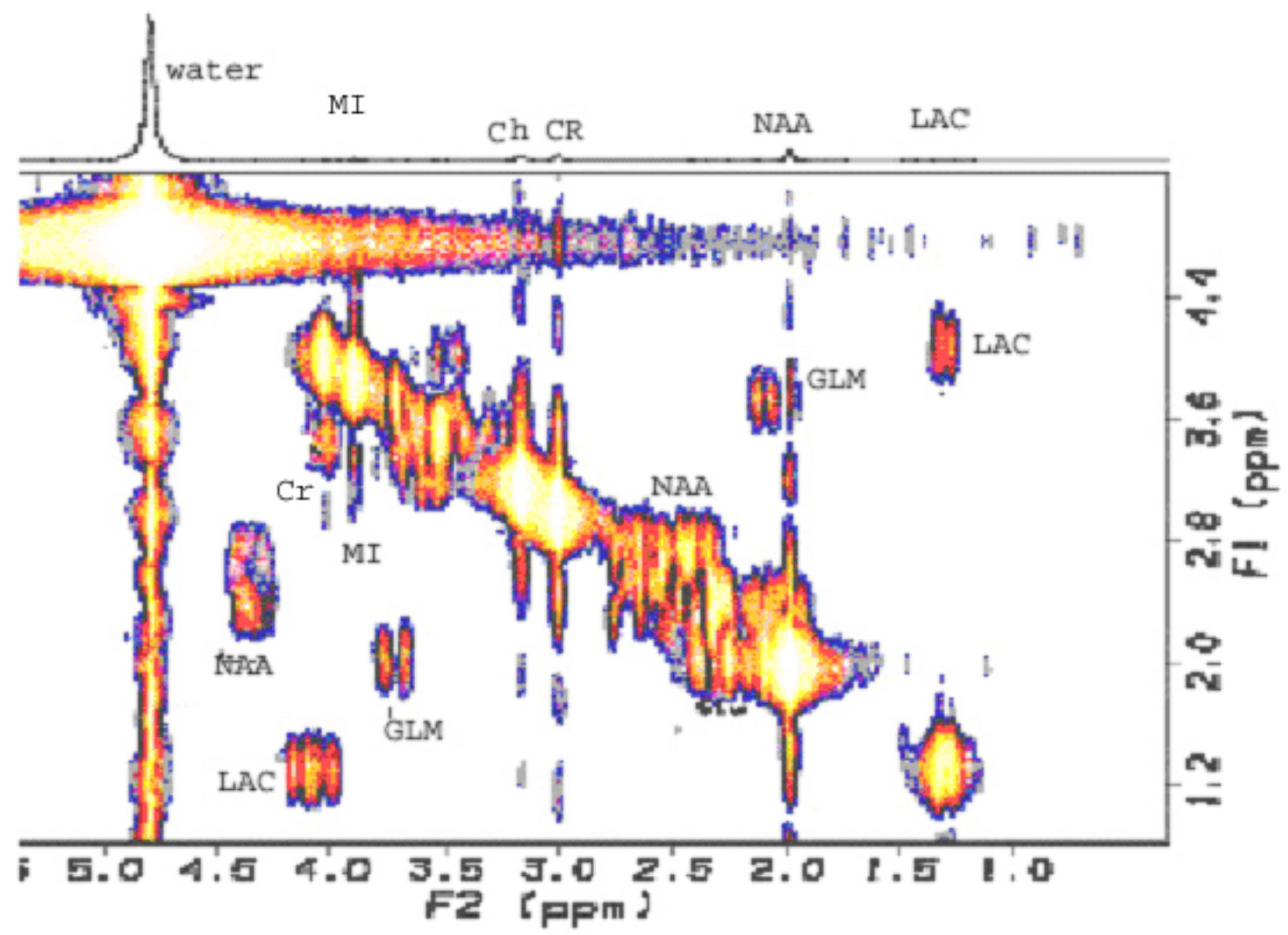

FIG. 2.21 2D L-COSY spectrum of a brain phantom

FIG. 2.21 shows the 2D L-COSY spectrum of a brain phantom. The pattern of the diagonal peaks at $\mathrm{F}_{2}=\mathrm{F}_{1}$ resembles the conventional 1D spectral resonances, whereas the cross peaks due to $J$-coupling between various protons of metabolites are observed on both sides of the diagonal peaks. 


\section{Chapter 3}

\subsection{Theoretical simulations of MRS by GAMMA}

GAMMA (General Approach to Magnetic Resonance Mathematical Analysis) is a magnetic resonance toolkit, based on the computer language $\mathrm{C}++$ written for simulations of MR experiments. Computer simulations of magnetic resonance experiments require a tedious programming step because standard computer languages do not include many features like quantum mechanical operators, super operators, tensors, and free induction decays. Many programs have been developed in recent years but the end user (spectroscopist) is not able to expand the language and introduce new features or new data types. An alternative approach for performing these MRS simulations using objectoriented language was proposed by Dr. Scott A. Smith and Tilo Levante [42].

GAMMA extends the computer language $\mathrm{C}++$ by adding data types used in magnetic resonance and all the data types describing operators, superoperators and tensors are realized in full analogy to the built in data types of a standard computer language. This permits end users to design their particular simulations in an abstract formalism analogous to the analytical description of the problem. Because each code is compiled before execution, it is time efficient for the GAMMA simulations of complex experiments on large spin systems. GAMMA programs are portable which makes the simulations of various multiple-pulse experiments of liquid state and solid state easy.

The layout of the GAMMA environment consists of three shells: mathematical shell, spectroscopic shell, and spectrometer operation shell.

The mathematical shell facilitates the quantum mechanical computations in operator formalism without explicit reference to the inherent matrix calculations in Hilbert space that represent the operator calculus. Relaxation and super operators are implicitly represented by matrix operations, in the Liouville Space. In many situations, it is not necessary to consider the entire density matrix, because certain elements are irrelevant for the selected observable. In such cases, the density operator can be expanded in terms of suitably chosen set of basis operators and to derive equations for their timedependent coefficients. These basis operators form the Liouville Space. 
The spectroscopic shell introduces higher-level concepts like the definition of a spin system and its associated Hamiltonian, the evolution of the density operator under the Hamiltonian, the effect of ideal and of selective pulses and also the definition of a relaxation super operator for a given relaxation mechanism.

The outermost spectrometer operation shell represents the user interface of an actual spectrometer system and can provide a convenient tool for testing pulse programs written for the spectrometer without the need to reformulate the operations in computer terminology. The spectrometer operation shell is spectrometer dependent and has not yet been implemented in GAMMA.

GAMMA uses many abstract data types that have defined algebraic properties and associated functions and are readily manipulated in any program. The introduction of abstract data types reflect the important features of magnetic resonance computations, thus making GAMMA a flexible and versatile programming environment. Abstract data types can be defined as a set of objects coupled with a set of functions that are defined on the objects.

GAMMA uses matrix data types such as diagonal, block diagonal, Hermitian, symmetric, and sparse matrices, derived from a basic class called matrix, which appear in magnetic resonance applications. The user deals with a single data type matrix but the implementation invokes the appropriate functions for the specific matrix types. A matrix in a Hilbert space spanned by a complete basis set represents an operator. The Hilbert space of the spin Hamiltonian is of finite dimensions and permits closed solutions for analysis of MR experiments. The Hilbert space defines the vector space of all admissible state functions. A Liouville space super operator is used for handling relaxation and chemical exchange in the coupled spin systems.

There are many functions that are specific to magnetic resonance. Listed below are areas for which functions are given.

Hamiltonians: Hamiltonians can be employed to describe the various interactions in the spin system such as the isotropic chemical-shift, $J$-coupling, dipolar-coupling, and quadrapolar-coupling.

Evolution: Using an appropriate function call the computation of a density matrix evolution can be obtained. The functions provided return a propagator for a specified 
evolution. Relaxation may be considered during the evolution. There are functions for ideal pulses, real pulses, and shaped pulses.

Acquisition: Functions for FID acquisition allow for a specified detection operator and time evolution under a static Hamiltonian with or without the effects of relaxation.

Data processing: Many routines such as windowing functions and Fourier transformation are used for data processing.

Input-output: There are provisions in GAMMA for data storage and retrieval by interfacing to other data processing (Felix, MRSi, Matlab) and text processing (FrameMaker) programs.

A spin system gives details of the number of spins involved, chemical shifts, J coupling and also the frequency at which the spectrometer is operating. A typical spin system used as an input to GAMMA is given below.

NSpins (0): $3 \quad$ - Number of Spins in the System

Iso (0) (2): $1 \mathrm{H} \quad$ - Spin Isotope Type

Iso (1) (2): $1 \mathrm{H} \quad$ - Spin Isotope Type

PPM (0) (1): $3.8914 \quad$-chemical shifts in ppm

PPM (1) (1): $2.8011 \quad$ - chemical shifts in ppm

PPM (2) (1): 2.6533

$\mathrm{J}(0,1)$ (1): $3.647 \quad$ - coupling constants in $\mathrm{Hz}$

Omega (1): $64 \quad$ - Spectrometer Frequency in $\mathrm{MHz}\left({ }^{1} \mathrm{H}\right.$ based)

A spin system file (demo.sys) and spectral parameters such as acquisition points along the $F_{1}$ and $F_{2}$ dimensions, spectral width is supplied. Mathematical variables like Hamiltonians and detectors are created and a pulse is applied on the channel to be detected. The program is executed using the GAMMA command at the shell prompt and it outputs the generated spectrum in ASCII (spec.asc). Gnuplot is used to produce a plot on the screen using the generated load file (spec.gnu).

\subsection{One-dimensional Simulation}

One-dimensional experiment uses a single radiofrequency pulse which tips the spins from their equilibrium position along the z-axis into $x-y$ plane, where they then precess at their characteristic frequencies determined by the chemical shifts and coupling 
constants. Such a system can be described using density matrix for the ensemble of spins. A product operator is used to keep track of the state of the spins i.e., the form of density matrix. The operators used form a complete basis set, so that any density matrix can be written as a sum of the basic operators [44].

We will now take the case where the spins $a$ and $b$ are coupled to each other with frequencies $v_{a}$ and $v_{b}$ and coupling constant $J$. When a $90^{\circ}$ pulse is applied along $y$ axis the magnetization which at equilibrium lies along the $z$-axis rotates the spins to the $x$-axis , where they precess about $z$-axis due to chemical shifts and couplings, giving a free induction decay (FID) with a signal at time $t$ : (only real magnetization components detected by spectrometer are considered)

$$
\begin{aligned}
& I_{a z}+I_{b z} \stackrel{90_{y}^{0}}{\longrightarrow} I_{a x}+I_{b x} \stackrel{\text { evolvetime } t}{\longrightarrow} I_{a x} \cos \left\{\left[v_{a}+(J / 2)\right] t\right\}+I_{a y} \sin \left\{\left[v_{a}+(J / 2)\right] t\right\} \\
& +I_{a x} \cos \left\{\left[v_{a}-(J / 2)\right] t\right\}+I_{a y} \sin \left\{\left[v_{a}-(J / 2)\right] t\right\} \\
& +I_{b x} \cos \left\{\left[v_{a}+(J / 2)\right] t\right\}+I_{b y} \sin \left\{\left[v_{a}+(J / 2)\right] t\right\} \\
& +I_{b x} \cos \left\{\left[v_{a}-(J / 2)\right] t\right\}+I_{b y} \sin \left\{\left[v_{a}-(J / 2)\right] t\right\}
\end{aligned}
$$

GAMMA contains functions, which will apply a pulse to a density operator representing a spin system, and functions, which will acquire transverse magnetization points during FID acquisition. The pulse can be of many types: ideal, rectangular, shaped, and composite. An ideal pulse is a good hard pulse that hits all selected spins evenly and is used in most of the programs. Relaxation is neglected for simplicity, but could be included as needed.

The program begins with set of queries, which prompt the user to supply needed parameter values. For ideal pulses there are no pulse lengths or offsets. The simplest acquisition one can perform in GAMMA is to use an FID function. The FID here will not decay because the system is evolving under an isotropic Hamiltonian that ignores relaxation effects. Thus we have to apply an apodization (convolve the time domain signal with a certain time domain function) prior to any FFT when processing the simulated FID. Next is application of an FFT and output the row vector (FFT) to an ASCII file, then call Gnuplot to make it plot the file.

The one-pulse experiment can be extended to simulate the effect of field strengths. Various metabolites are simulated at different field strengths $(1.5 \mathrm{~T} / 3 \mathrm{~T})$ to 
understand spectral patterns of different metabolites. The simulation results for the following metabolites are shown below. All the simulations were performed with 512 acquisition points and line broadening of $1 \mathrm{~Hz}$. We observe that the spectrum of metabolite is much resolved at higher magnetic fields. This is due to the increase in spectral dispersion leading to higher resolution. Protons at $1.5 \mathrm{~T}$ field strength resonate at $64 \mathrm{MHz}$, whereas protons at 3.0T field strength resonate at $128 \mathrm{MHz}$. The simulations are plotted amplitude Vs chemical shift (PPM).

\section{ACETATE:}

${ }^{2} \mathrm{CH}_{3}-{ }^{1} \mathrm{COO}^{-}$

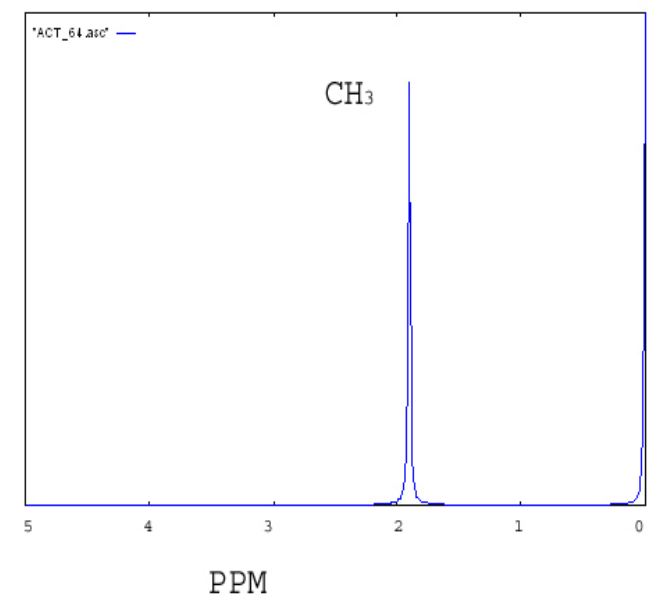

FIG. 3.0.0 Theoretical simulation of Acetate at $1.5 \mathrm{~T}$.

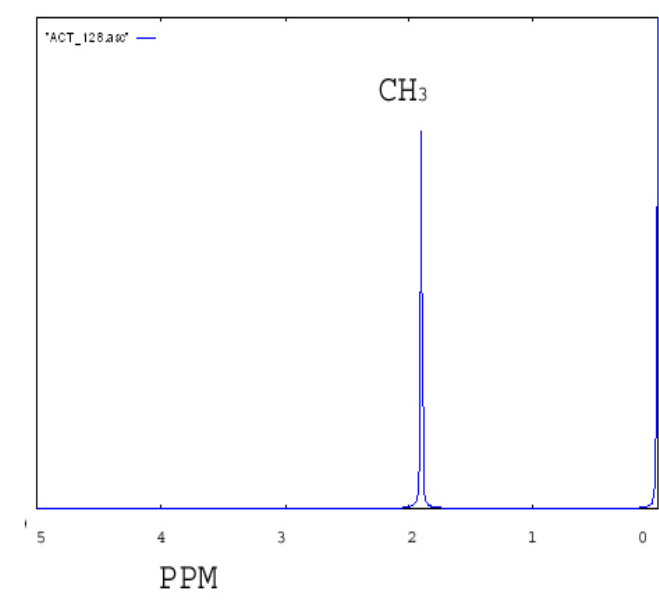

FIG. 3.0.1.Theoretical simulation of Acetate at $3.0 \mathrm{~T}$.

FIG. 3.0.0 and FIG. 3.0.1 shows the theoretical simulation of Acetate at 1.5T and 3.0T field strength respectively. 
ASPARTATE:

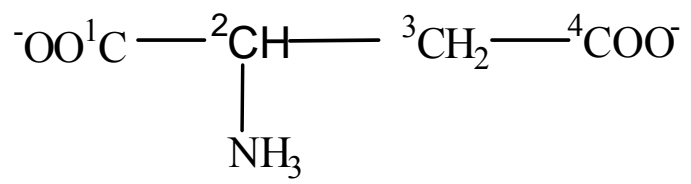

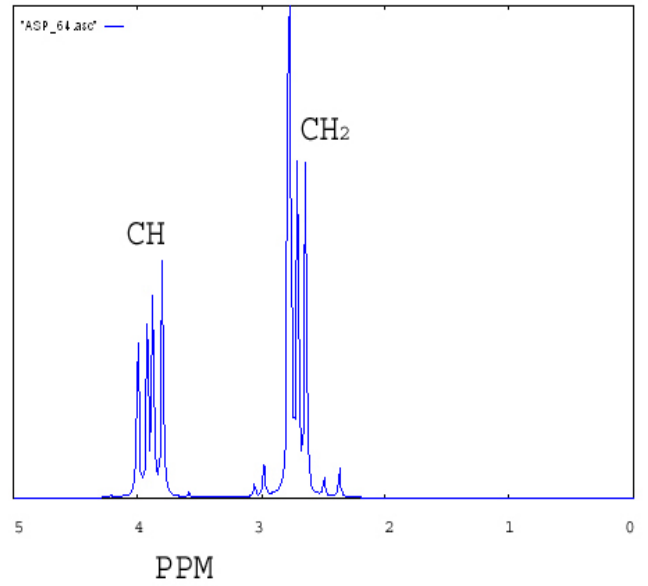

FIG. 3.0.3 Theoretical simulation of Aspartate at $1.5 \mathrm{~T}$.

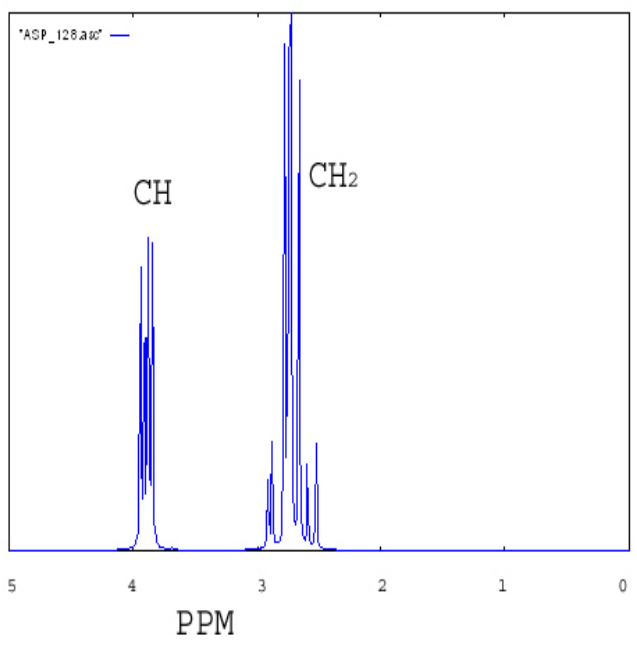

FIG. 3.0.4. Theoretical simulation of Aspartate at $3.0 \mathrm{~T}$.

FIG. 3.0.3 shows the spectrum of Aspartate at 1.5T field strength, whereas FIG. 3.0.4 shows the spectra at 3.0T field strength. The spectral lines in the ranges 2-3 ppm and 3-4 ppm are much resolved in the spectrum simulated at 3.0T. We can observe that the spectrum at 3.0T is highly resolved than the spectrum at $1.5 \mathrm{~T}$ field strength. 


\section{CREATINE:}

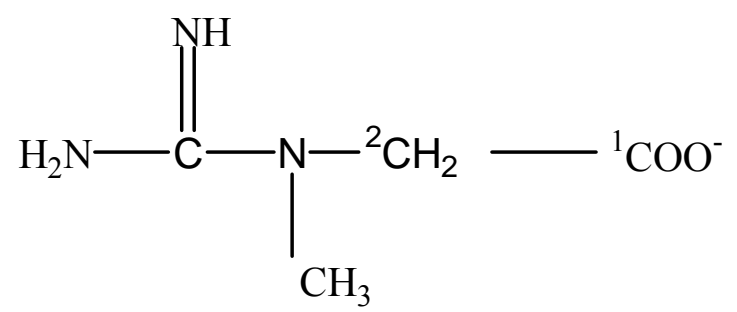

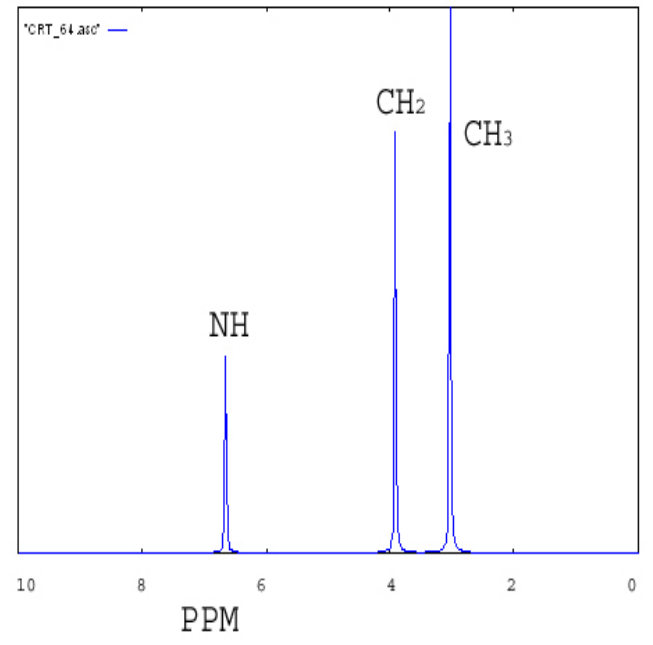

FIG. 3.0.5 Theoretical simulation of Creatine at $1.5 \mathrm{~T}$.

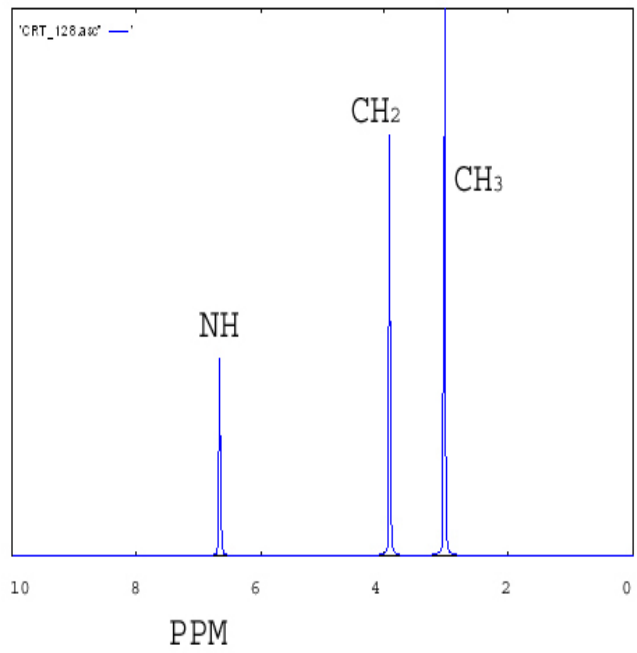

FIG. 3.0.6 Theoretical simulation of Creatine at $3.0 \mathrm{~T}$.

The theoretical simulations of creatine at 1.5T and 3.0T field strength are shown in FIG. 3.0.5 and FIG. 3.0.6 respectively. The protons resonating at different frequencies give rise to three singlets. 


\section{GABA:}

$\mathrm{NH}_{3}-{ }^{4} \mathrm{CH}_{2}-{ }^{3} \mathrm{CH}_{2}-{ }^{2} \mathrm{CH}_{2}-{ }^{1} \mathrm{COO}^{-}$

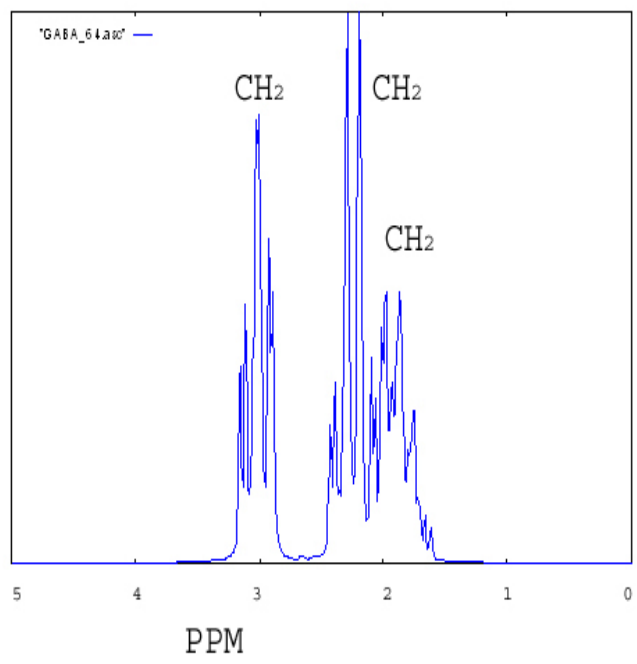

FIG. 3.0.7 Theoretical simulation of GABA at $1.5 \mathrm{~T}$.

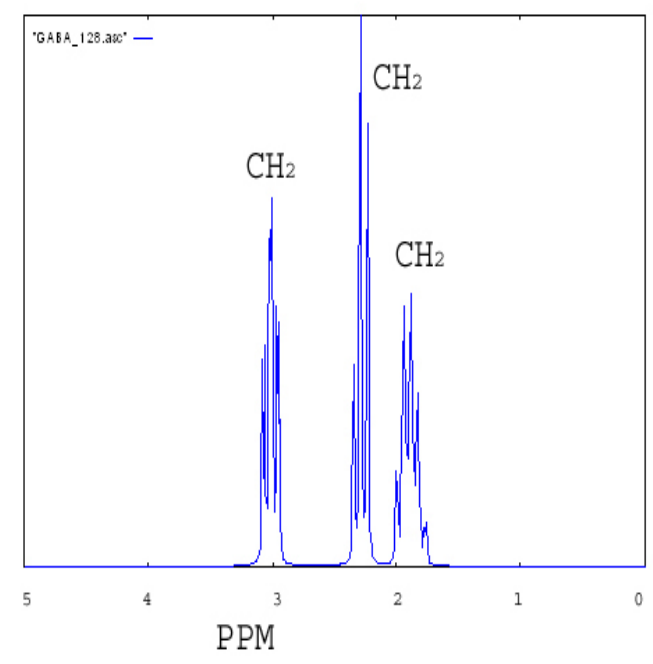

FIG. 3.0.8 Theoretical simulation of GABA at $3.0 \mathrm{~T}$.

The spectrum obtained by the theoretical simulations of GABA at 3.0T (FIG. 3.0.7) shows highly resolved spectral lines compared to spectrum obtained at 1.5T (FIG. 3.0.8). The spectral lines which are overlapped in the spectrum obtained at $1.5 \mathrm{~T}$ field strength are well resolved in the spectrum obtained at 3.0T field strength. 


\section{GLUTAMATE:}

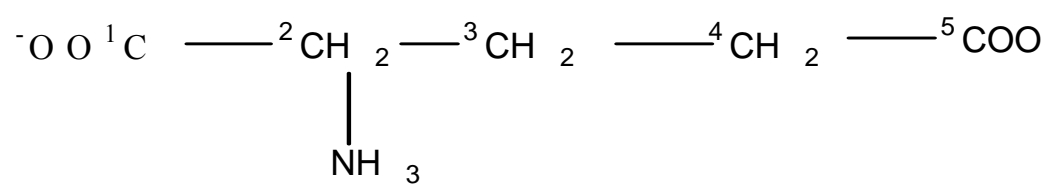

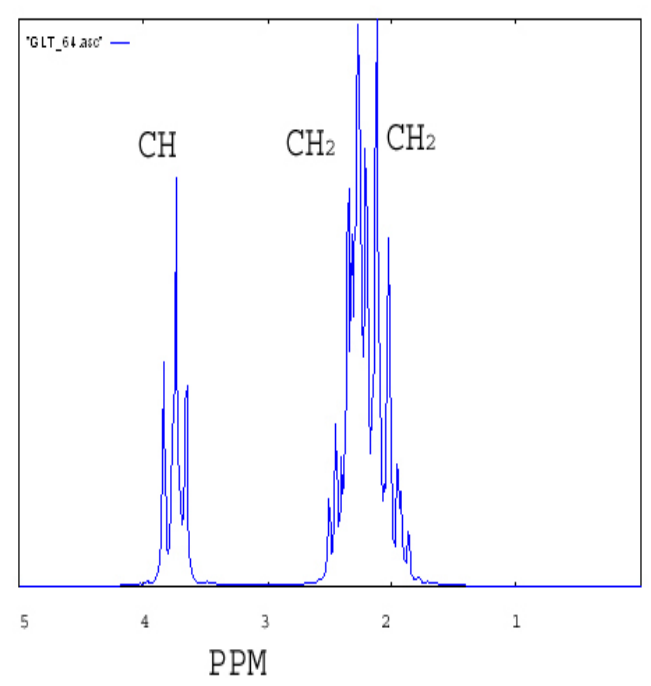

FIG. 3.0.9 Theoretical simulation of Glutamate at $1.5 \mathrm{~T}$.

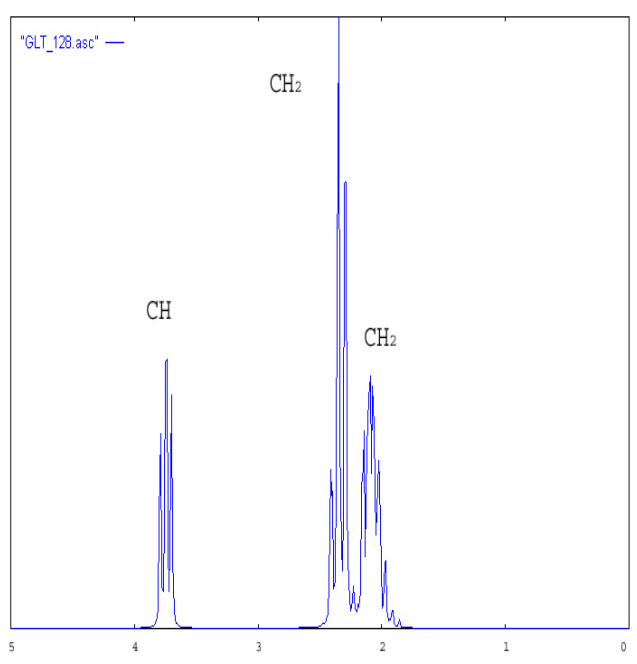

FIG. 3.0.10 Theoretical simulation of Glutamate at $3.0 \mathrm{~T}$.

FIG. 3.0.9 shows the spectrum obtained by the theoretical simulations of Glutamate at 1.5T field strength. We can observe the spectral lines are overlapped in the range of 1.52.5 ppm. The spectrum obtained at 3.0T (FIG. 3.0.10) shows highly resolved spectral lines. 
GLUTAMINE:

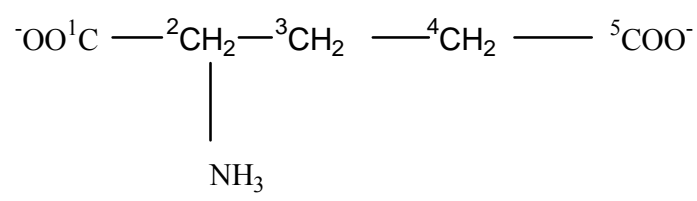

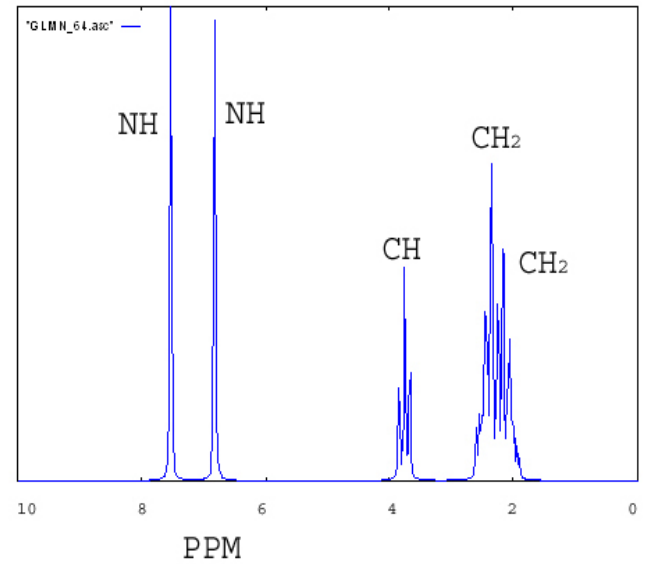

FIG. 3.0.11 Theoretical simulation of Glutamine at $1.5 \mathrm{~T}$.

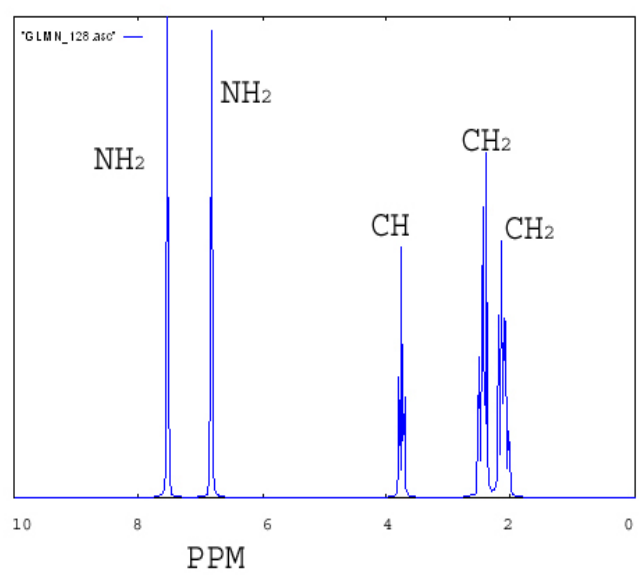

FIG. 3.0.12 Theoretical simulation of Glutamine at $3.0 \mathrm{~T}$.

The effect of field strength on the spectral lines can be better understood by looking at the spectrum obtained by simulations of Glutamine at $1.5 \mathrm{~T}$ and 3.0T field strengths. The spectral lines in the range 2-4 ppm are better resolved at 3.0T (FIG. 3.0.11) whereas they are overlapped in spectrum at 1.5T (FIG. 3.0.12). 


\section{LACTATE:}

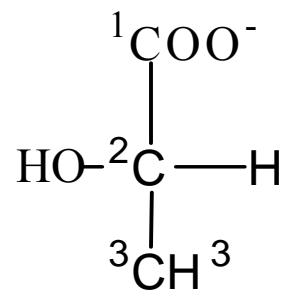

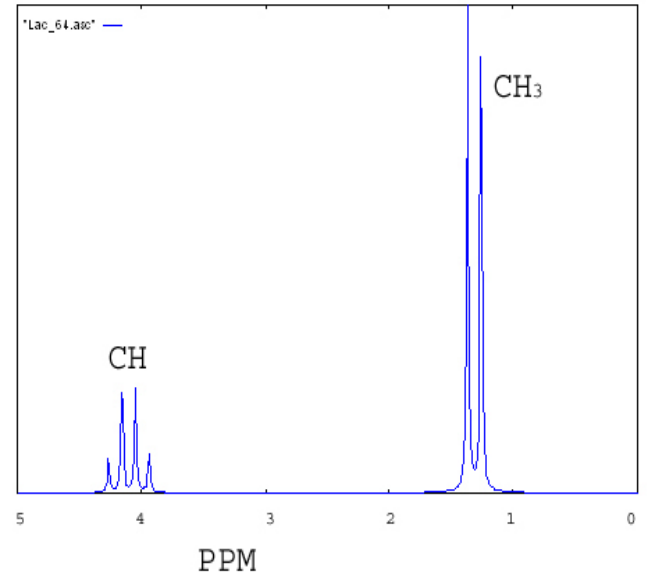

FIG. 3.0.13 Theoretical simulation of Lactate at $1.5 \mathrm{~T}$.

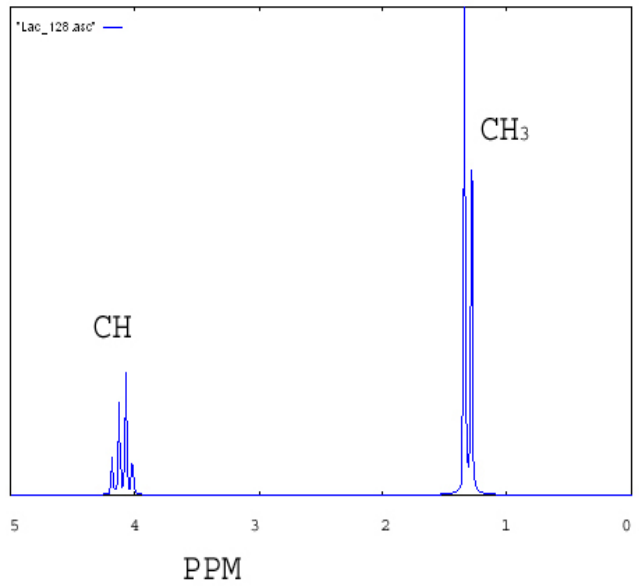

FIG. 3.0.14 Theoretical simulation of Lactate at $3.0 \mathrm{~T}$.

The spectrum of Lactate obtained at 1.5T and 3.0T are shown in FIG. 3.0.13 and FIG. 3.0.14 respectively. 


\section{MIO-INOSITOL:}

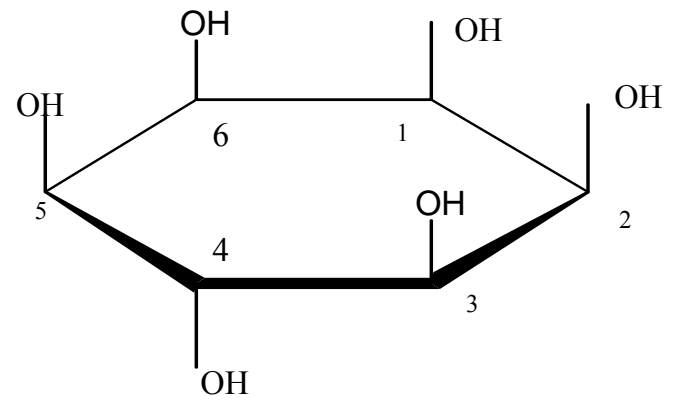

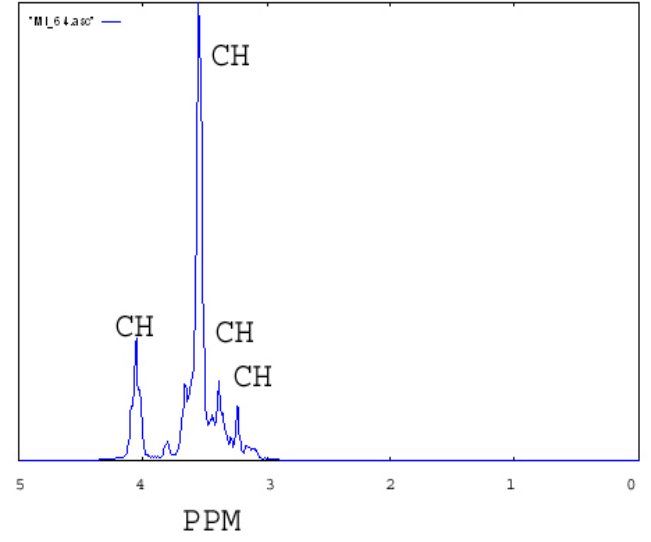

FIG. 3.0.15 Theoretical simulation of MioInositol at $1.5 \mathrm{~T}$.

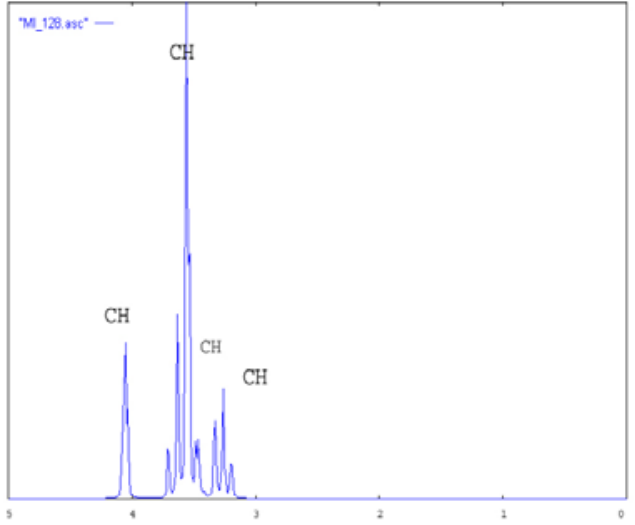

FIG. 3.0.16 Theoretical simulation of MioInositol at 3.0T.

The spectrum of Mio-Inositol obtained at 1.5T and 3.0T are shown in FIG. 3.0.15 and FIG. 3.0.16 respectively. The spectral lines in the spectrum of Mio-Inositol at 3.0T are better resolved than in the spectrum at $1.5 \mathrm{~T}$. 


\section{PHOSPHO-CREATINE:}<smiles>CN([CH]C(=O)[O-])C(=N)NP(=O)([O-])[O-]</smiles>

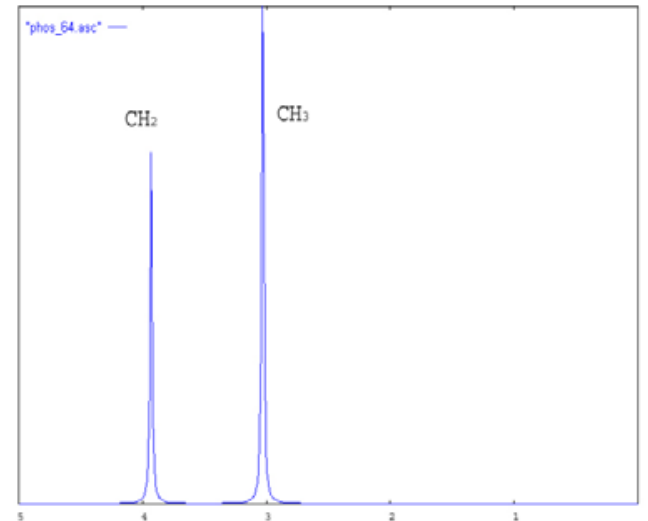

FIG. 3.0.17 Theoretical simulation of PhosphoCreatine at $1.5 \mathrm{~T}$.

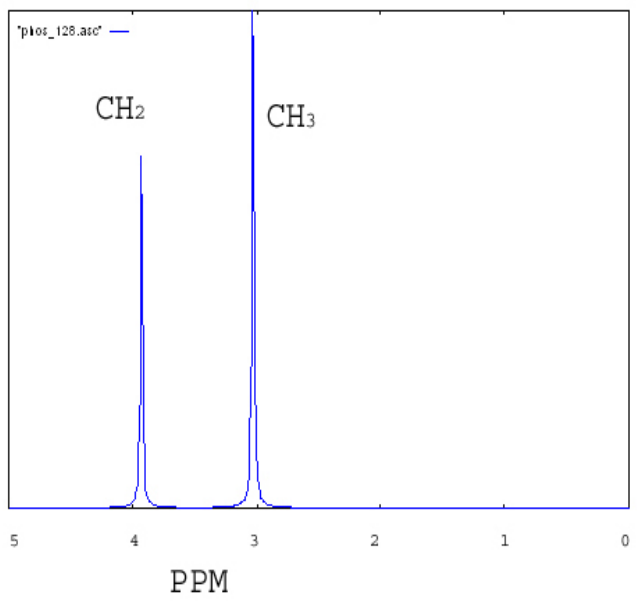

FIG. 3.0.18 Theoretical simulation of PhosphoCreatine at 3.0T.

FIG. 3.0.17 and FIG. 3.0.18 show the theoretical simulations of Phospho-Creatine at 1.5T and 3.0T field strengths respectively.

\subsection{Two-dimensional Experiments:}

Two-dimensional MRS experiments can be simulated in the same straightforward manner as they are performed by the spectrometer. The general idea of $2 \mathrm{D}$ experiments is to add a delay and at least one more pulse before the system return to equilibrium. In COSY experiment a second pulse is added after delay period (called $t_{1}$ ). An algorithm for a $2 \mathrm{D}$ COSY experiment could read as follows:

Read parameters from the user input and/or from files

Set-up pulse Hamiltonians, propogators, initial density operator, detection operator, time increments Apply a $90^{\circ}$ pulse for each $\mathrm{t}_{1}$ point do 


\section{Evolve over $t_{1}$ increments}

Apply $180^{\circ}$ pulse

Evolve over $t_{1}$ time

Apply $90^{\circ}$ pulse

Perform an acquisition

Perform apodization

Apply FFT

Increment $\mathrm{t}_{1}$ delay

End of loop

Apply second FFT

Output to ascii file

call gnuplot

In this case, the value of $t_{1}$ is a parameter and a complete two-dimensional data set consists of $N$ FIDs (signals as a function of the time $\mathrm{t}_{2}$ ), which depends on the value of $\mathrm{t}_{1}$ $\left(\mathrm{t}_{1}=\mathrm{t}_{1}+\mathrm{n} \Delta \mathrm{t}_{1}\right.$, and $\left.n=1,2,3 \ldots N\right)$. During $\mathrm{t}_{2}$ only terms containing some parts of the operator $I_{x}$ or $I_{y}$ can be detected. This means that the spectrum obtained by Fourier transforming with respect to $t_{2}$ contains lines whose positions correspond to those in an ordinary spectrum, but their amplitudes and phases depend on the value of $t_{1}$. At a particular frequency in each such spectrum, a plot of the amplitude at that spectral point as a function of the variable $t_{1}$ will produce a second FID. A second Fourier transformation of this FID results in a two-dimensional spectrum.

To predict the locations of peaks in the 2D spectrum, we can calculate the response of the spins to the pulses and the delays in the experiment. If the spins are not coupled to one another, then all magnetization remains on the same spin (and hence the same frequency) during both time periods. Thus, the peaks occur on the diagonal $v_{1}=v_{2}$.

In the case of coupled spins, we use EQ. 3.3 (replacing $t$ with $t_{1}$ ) to describe the state of the spins up to the second pulse, and carry out the rotations induced by the second $90^{\circ} \mathrm{y}$ pulse to determine the state of the spins immediately after the second pulse [44]:

$$
\begin{aligned}
& \left\{-I_{a z} \cos \left\{[(J / 2)] t_{1}\right\}+2 I_{a y} I_{b x} \sin \left\{[(J / 2)] t_{1}\right\} \cos \left(v_{a} t_{1}\right)\right. \\
& +\left\{+I_{a y} \cos \left\{[(J / 2)] t_{1}\right\}+2 I_{a z} i_{b x} \sin \left\{[(J / 2)] t_{1}\right\} \sin \left(v_{a} t_{1}\right)\right. \\
& +\left\{-I_{b z} \cos \left\{[(J / 2)] t_{1}\right\}+2 I_{b y} I_{a x} \sin \left\{[(J / 2)] t_{1}\right\} \cos \left(v_{b} t_{1}\right)\right. \\
& +\left\{+I_{b y} \cos \left\{[(J / 2)] t_{1}\right\}+2 I_{b z} I_{a x} \sin \left\{[(J / 2)] t_{1}\right\} \sin \left(v_{b} t_{1}\right)\right.
\end{aligned}
$$


Each of these terms again evolve under the influence of both chemical shifts and couplings during the detection period $t_{2}$. The evolution during the time period $t_{2}$ :

$$
\begin{aligned}
& 2 I_{a z} I_{b z} \stackrel{\text { evolvet }}{\longrightarrow} 2 I_{a z} I_{b x} \cos \left[(J / 2) t_{2}\right] \cos \left(v_{b} t_{2}\right)+2 I_{a z} I_{b y} \cos \left[(J / 2) t_{2}\right] \sin \left(v_{b} t_{2}\right) \\
& +I_{b y} \sin \left[(J / 2) t_{2}\right] \cos \left(v_{b} t_{2}\right)-I_{b x} \sin \left[(J / 2) t_{2}\right] \sin \left(v_{b} t_{2}\right)
\end{aligned}
$$

The first two terms are detectable $\left(I_{j z} I_{k x}\right.$ is detectable because it becomes $I_{k x}$ and $I_{k y}$ during $t_{2}$ ), and when we combine these with the evolution terms from the first time period EQ. [2] we obtain

$$
\begin{aligned}
& \left\{I_{b y} \sin \left[(J / 2) t_{2}\right] \cos \left(v_{a} t_{2}\right)-I_{b x} \sin \left[(J / 2) t_{2}\right] \sin \left(v_{b} t_{2}\right)\right\} \sin \left[(J / 2) t_{1}\right] \sin \left(v_{a} t_{1}\right)+ \\
& \left\{I_{a y} \sin \left[(J / 2) t_{2}\right] \cos \left(v_{a} t_{2}\right)-I_{a x} \sin \left[(J / 2) t_{2}\right] \sin \left(v_{a} t_{2}\right)\right\} \sin \left[(J / 2) t_{1}\right] \sin \left(v_{b} t_{1}\right)
\end{aligned}
$$

An observation of the other terms in EQ. (2.5) shows that some correspond to diagonal peaks (evolving at the same frequency during $t_{1}$ and $t_{2}$ ), while those in Eq. (3.7) correspond to cross peaks (different frequencies during these two periods). There are two cross peaks, each with multiplet structure: the first at $v_{a}$ in the $F_{l}$ dimension and $v_{b}$ in the $F_{2}$ dimension: and the second at $v_{b}$ in the $F_{1}$ dimension and $v_{a}$ in the $F_{2}$ dimension.

It is the coupling between the spins that allows magnetization to be transferred by the second pulse; that is cross peaks occur between pairs of spins only when they are directly coupled to one another. All directly coupled spins give rise to cross peaks at the intersection of their frequencies, regardless of the number of other spins that are coupled to either one. All the pulses used are ideal pulses. Gnuplots of 2D COSY for various metabolites are shown below obtained at 3.0T field strength. 


\section{CREATINE:}

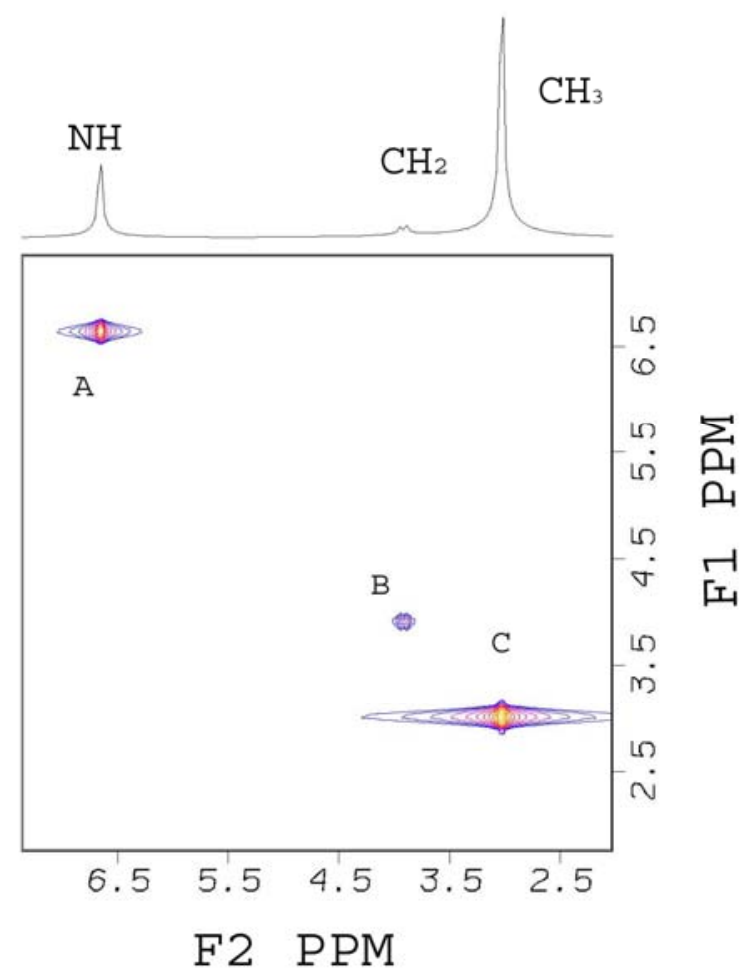

FIG. 3.1 2D COSY spectrum of Creatine

The spectrum shown in FIG. 3.1 is a 2D COSY plot for Creatine. We can observe the three singlet (A, B, C at 3.0270, 3.9130, $6.6490 \mathrm{ppm}$ ) due to $\mathrm{NH}, \mathrm{CH}_{2}$ and $\mathrm{CH}_{3}$ protons respectively which are on diagonal axis. There are no cross peaks since the protons from these groups are coupled to each other. 
GABA:

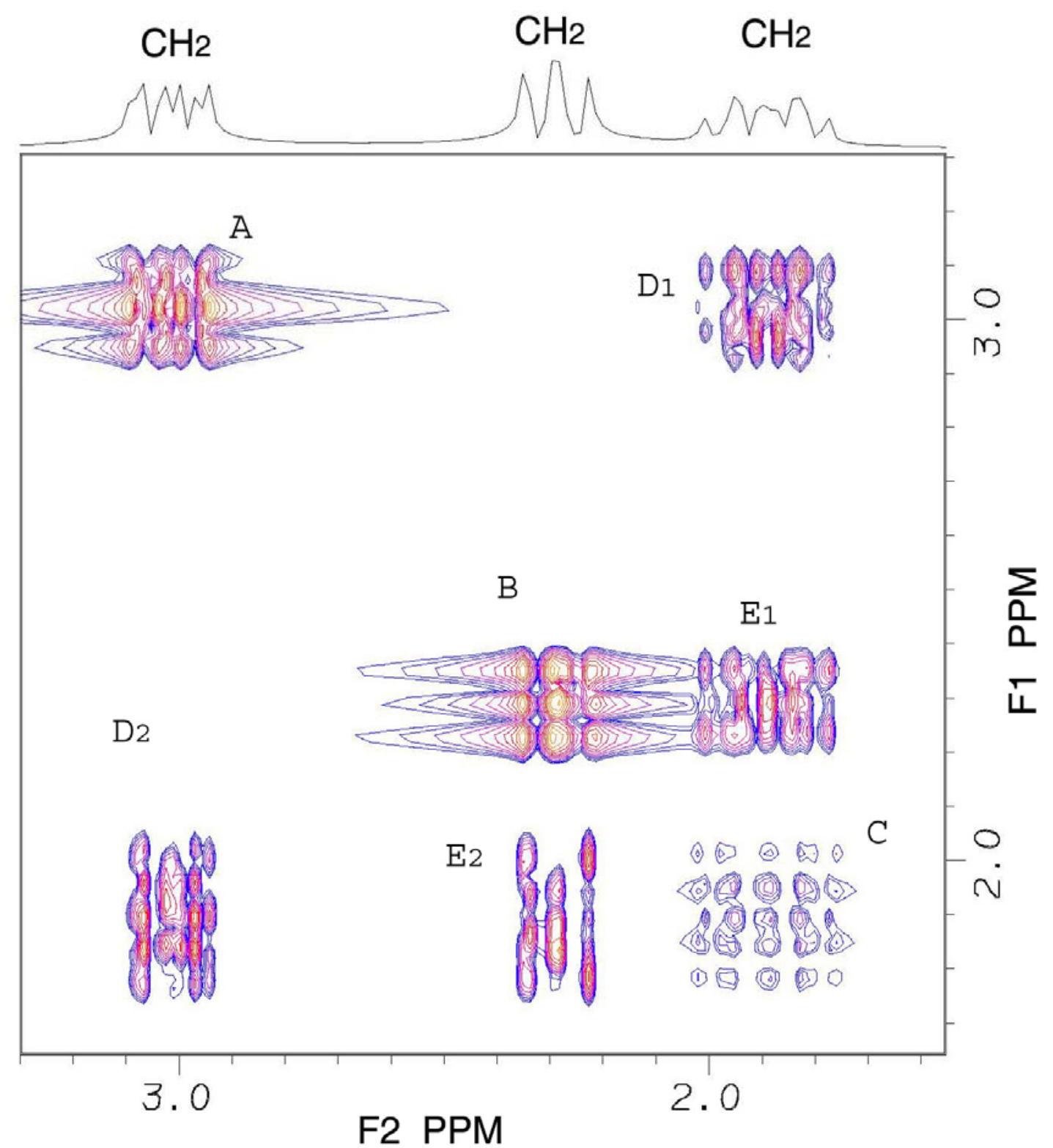

FIG. 3.2 2D COSY spectrum of GABA

The 2D COSY spectrum of GABA is shown in FIG. 3.2. The diagonal peaks (A, B, C) show the chemical shifts of the three $\mathrm{CH}_{2}$ groups of GABA resonating at 1.8890, 2.2840, $3.0128 \mathrm{ppm}$ respectively. Since the protons are coupled to each other we observe cross peaks at $3.0128 \mathrm{ppm}\left(\mathrm{D}_{2}\right), 1.8890 \mathrm{ppm}\left(\mathrm{D}_{1}\right.$ and $\left.\mathrm{E}_{1}\right)$ and $2.2840 \mathrm{ppm}(\mathrm{E} 2)$. 


\section{GLUTAMATE:}

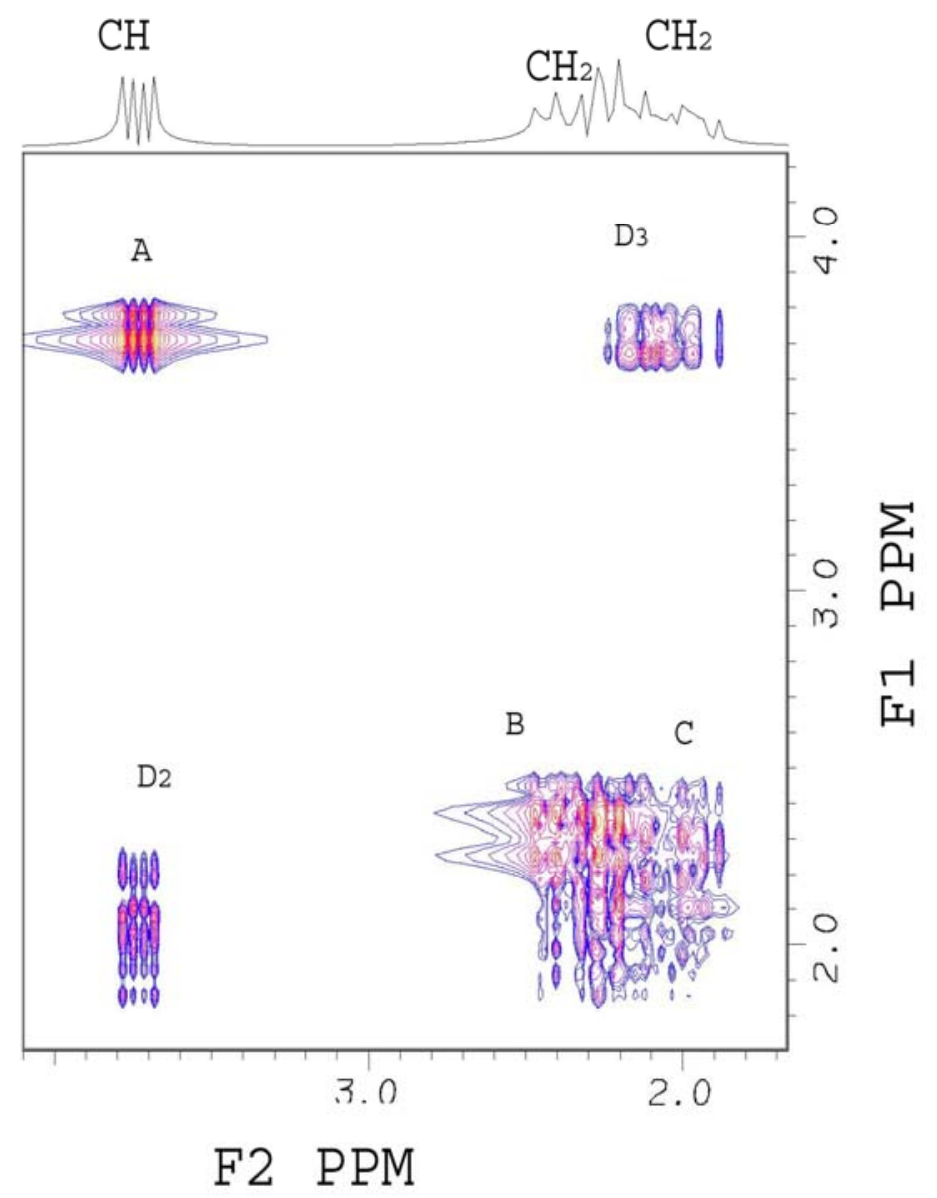

FIG. 3.3 2D COSY spectrum of Glutamate

Glutamate has a complex structure and many coupled protons. This results in a complex spectrum where cross peaks and diagonal peaks are overlapping. It has five protons resonating at 3.7433, 2.1290, 2.1090, 2.4320, 2.4540 ppm (FIG. 3.3) respectively coupled with each other. The diagonal peaks are labeled as A, B, C. Since two of the protons resonate at very close $(2.1290$ and $2.1090 \mathrm{ppm})$ we see overlap of diagonal peaks. We can also observe the cross peaks at $3.7433 \mathrm{ppm}\left(\mathrm{D}_{2}\right)$ and $2.0375 \mathrm{ppm}\left(\mathrm{D}_{3}\right)$. 


\section{LACTATE:}

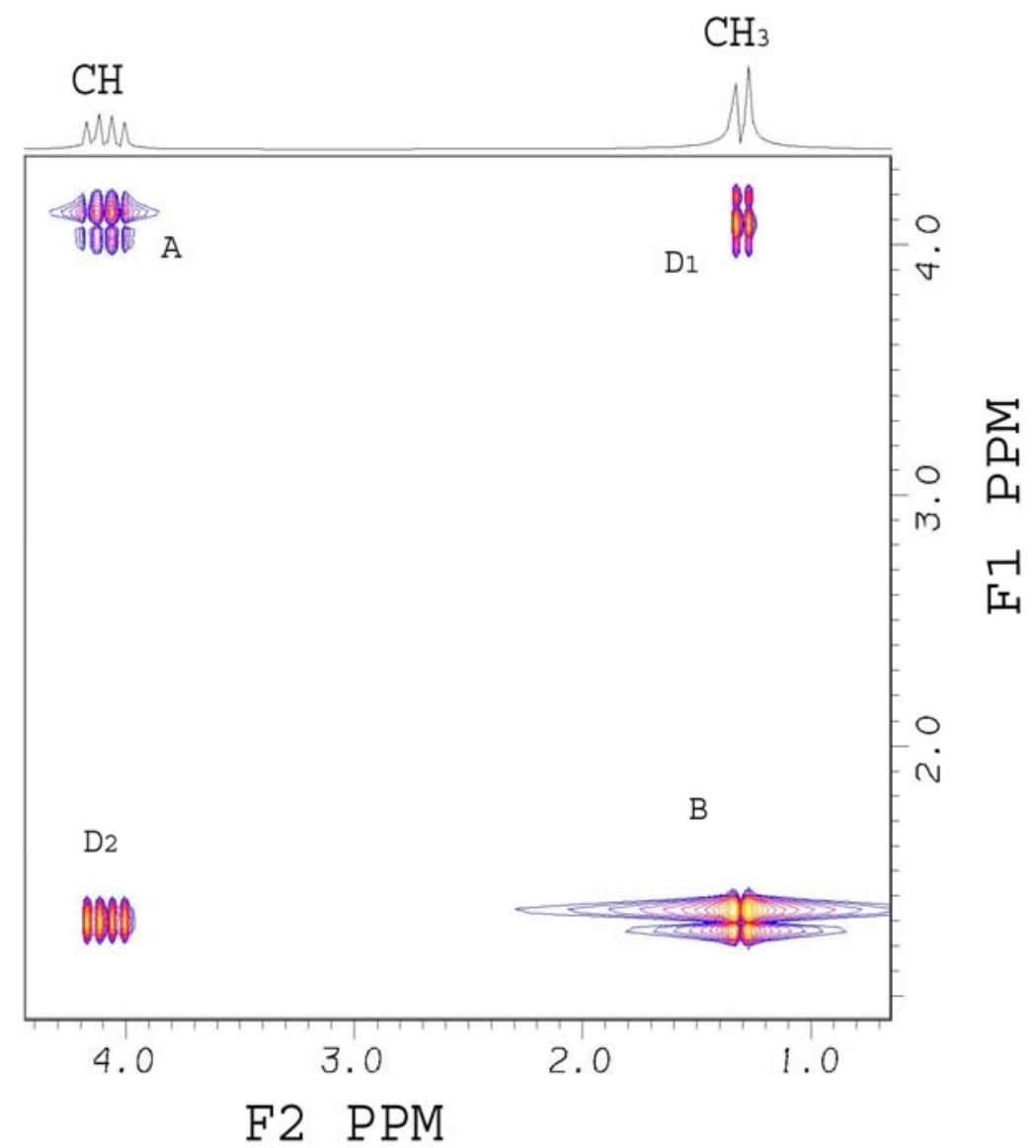

FIG. 3.4 2D COSY Spectrum of Lactate

FIG. 3.4 shows 2D COSY spectrum of Lactate. Lactate has a $\mathrm{CH}_{3}$ and $\mathrm{CH}$ group resonating at $1.3142 \mathrm{ppm}$ (B) and $4.0974 \mathrm{ppm}$ (A) respectively. We see the diagonal peaks at $4.0974 \mathrm{ppm}(\mathrm{A})$ and 1.3142 (B) ppm. These protons are coupled to each other and give rise to cross peaks 4.0974 ppm (D1) and 1.3142 ppm (D2). 


\section{MIO-INOSITOL:}

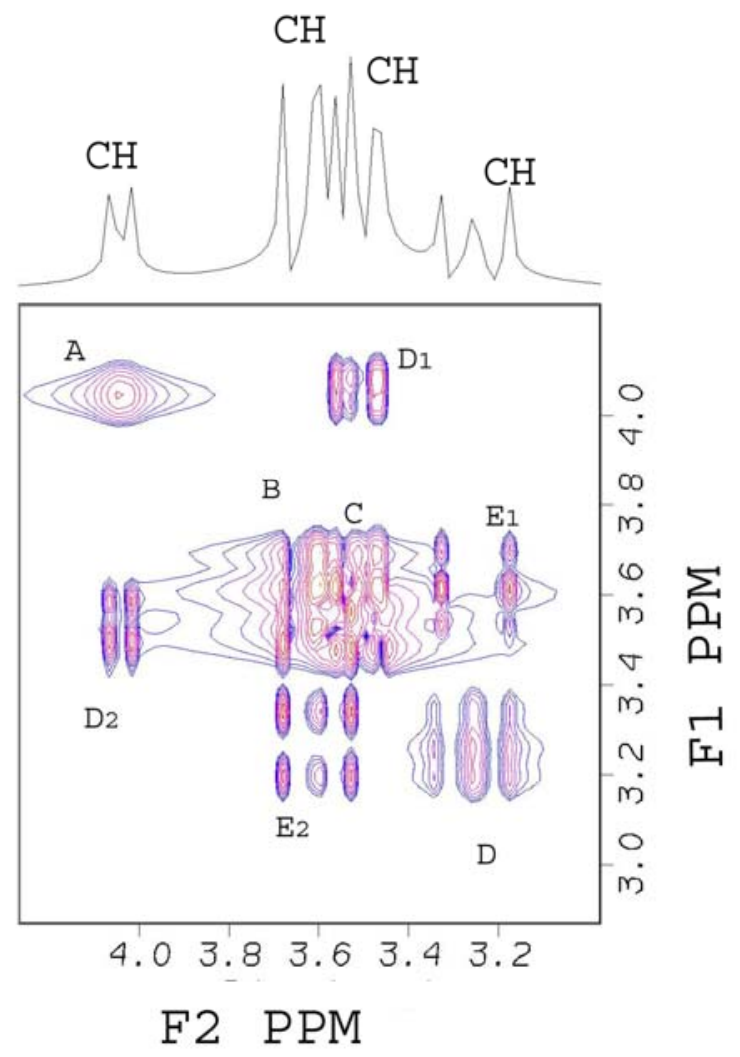

FIG. 3.5 2D COSY spectrum of Myo-Inositol

The 2D COSY spectrum of Mio-Inositol is shown in FIG. 3.5. Myo-Inositol has six CH groups. Six protons resonate 3.5217 ppm (C), 3.6144 ppm (B), 4.0538 ppm (A), 3.2690 ppm (D). We see that the diagonal peaks $\mathrm{B}$ and $\mathrm{C}$ are overlapping as their resonating frequencies are close. Cross peaks are seen which resonate at $4.0538 \mathrm{ppm}\left(\mathrm{D}_{2}\right), 3.6144$ ppm ( $\left.\mathrm{E}_{2}\right), 3.5217 \mathrm{ppm}\left(\mathrm{D}_{1}\right), 3.2690 \mathrm{ppm}\left(\mathrm{E}_{1}\right)$. 


\section{Chapter 4}

\subsection{Development of Constant-Time Sequence}

In this chapter we describe the design and implementation of our new Localized Constant-Time Correlated technique on a GE 3T MRI/MRS scanner.

\subsubsection{Current 2D MRS approach}

L-COSY (Localized COrrelated SpectroscoY), a 2D MRS technique [41], can be used to record single voxel localized 2D proton spectra in vitro as well as in vivo. Fig. 4.1 shows the 2D L-COSY technique that can unambiguously resolve many overlapping peaks, improve spectral resolution and also achieve better dispersion of several metabolites. FIG. 4.1 shows the three pulses in time, as $\Delta \mathrm{t}_{1}$ is incremented.

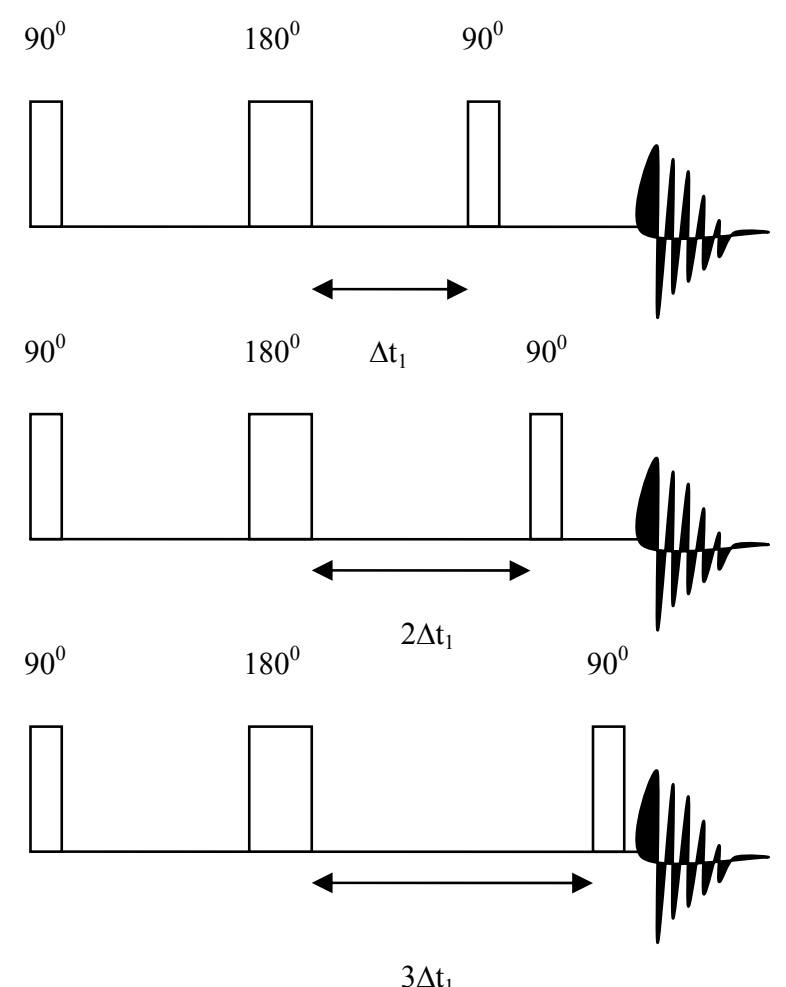

FIG. 4.1 2D LCOSY experiment

From FIG. 4.1, we can observe that the time between the first $90^{\circ}$ pulse and second $90^{\circ}$ pulse changes during each increment. 
But 2D L-COSY has some limitations such as

1. Since the time delay between the $180^{\circ}$ pulse and second $90^{\circ}$ pulse is incremented for each experiment, we see a change in total echo time during the $2 \mathrm{D}$ experiment. This change in time results in variable coherence transfer between spins that are connected through $J$-coupling.

2. Due to systematic increment in time delay during the $2 \mathrm{D}$ experiment the echo time and repetition time for each increment changes. Therefore we see a change in $\mathrm{T}_{1}$ and $\mathrm{T}_{2}$ relaxations for each increment. This variation in relaxation periods complicates the quantification of metabolites.

3. The $J$ modulation in this $2 \mathrm{D}$ experiment reduces the sensitivity of the cross peaks.

4. Though most of the overlapping peaks are resolved still there is considerable overlap of cross peaks for some important metabolites.

To address the above issues we have developed the technique for a 2D Constant-time Correlated Spectroscopic experiment, which has a fixed interval $t_{1}$ during the evolution period.

\subsubsection{Design of Constant Time experiment:}

Every two-dimensional experiment begins with a preparation period, which may be considered as a relaxation delay for generating equilibrium or steady state [21]. Preparation ends with an excitation (e.g., a $90^{\circ}$ pulse) to create $x y$ magnetization or to create a specific initial state of the spin system, which then evolves during $t_{1}$. The evolution time $\mathrm{t}_{1}$ is incremented in equal steps $\Delta \mathrm{t}_{1}$ corresponding to a spectral width $S W_{1}$. Separate data acquisitions are performed for each value of $\mathrm{t}_{1}$ to give N1 FIDs, each with N2 data points. Depending on the nature of the mixing period, generally initiated by an RF pulse, and the interactions present between spins, signal intensities and/or phases detected during $t_{2}$ will be modulated in a periodic way, as $t_{1}$ is incremented. In simple terms, if a component of magnetization precessing with frequency $F_{l}$ during $\mathrm{t}_{1}$ influences or is transferred to a component precessing at $F_{2}$ during $\mathrm{t}_{2}$, then correlation signals with coordinates $\left(F_{1}, F 2\right)$ appear in the 2D data matrix.

The type of $2 \mathrm{D}$ experiment to be performed is determined by the exact details of how preparation, evolution, and mixing periods are constructed. A preparation period establishes the condition of the spin system at the beginning of $t_{1}$. It usually ends with a 
pulse that generates transverse magnetization. The evolution period is generally a period of free precession for transverse magnetization, during which the various magnetization components evolve in time and become distributed in the $x y$ plane according to their respective frequencies. Evolution may be influenced by spin decoupling, by $180^{\circ}$ refocusing pulses, or by other perturbations such as sample spinning or gradient pulses. The mixing period may include one or more pulses and delays that cause a mixing of spin states between interacting spins. The effects of mixing are reflected in changes in the amplitudes and/or phases of the detected signals and depend on the phase encoding, which varies periodically with $\mathrm{t}_{1}$.

Evolution $\left(\mathrm{T}_{\mathrm{ct}}+\mathrm{t}_{1} / 2\right)-\left[180^{0}\right]$-evolution $\left(\mathrm{T}_{\mathrm{ct}}-\mathrm{t}_{1} / 2\right)$-mixing

The above expression represents a more specialized application in which the total time for the evolution period $\mathrm{T}_{\mathrm{ct}}$ is kept constant and the position of the $\left[180^{\circ}\right]$ pulse is stepped through this period. When $t_{1}=t_{0}$, chemical shifts show maximum negative evolution, which decreases toward zero and becomes positive (increases in a positive sense) as $t_{1}$ is incremented and the $\left[180^{\circ}\right]$ shifts through the centre and out towards the end of the $T_{c t}$ period. At the same time, couplings that are unaffected by $\left[180^{\circ}\right]$ contribute only a constant phase factor and do not act as modulations in the $t_{1}$ domain.

\subsubsection{Refocusing pulses:}

A pulse of the RF field long enough to tip the spin vector through exactly $90^{\circ}$ is called a $90^{\circ}$ pulse. A $90^{\circ}$ pulse is used to rotate the magnetization into the $x y$ plane. Further pulses can act on the initial pulse; for example another $90^{\circ}$ pulse will place the magnetization on the $z$-axis, and using a $180^{\circ}$ pulse, the magnetization will precess to a new position in the $x y$ plane. The new direction of the magnetization is the mirror image of its starting position with respect to the axis used for the pulse. The $180^{\circ}$ pulse employs a $90^{\circ}$-phase shift of the RF frequency. Many NMR experiments utilize the elementary sequence $\left[90^{\circ}-\tau-180^{\circ}-\tau\right]$ as the primary building block within a pulse sequence ( $\tau$ is a delay time). A simple system with two independent spin vectors can be considered to examine the effect of this part of pulse sequence. After a $90^{\circ}$ pulse the two vectors lie in the $x y$ plane, one precessing at a faster rate $(\mathrm{F})$ than the other $(\mathrm{S})$. When an $180^{\circ}$ refocusing pulse along the $y$-axis is applied, $\mathrm{B}_{1}$ precession of the individual spin vectors is forced to new positions within the $x y$ plane, after which normal $\mathrm{B}_{0}$ precession 
continues. The spins are once again aligned along y-axis at time $2 \tau$ following the beginning of the sequence, since precisely the same distance is covered by, for example the $\mathrm{F}$ spin as it had traveled before the $180^{\circ}$ pulse. There is no variation of peak intensities as a function of the length of the refocusing period since all spins are refocused at the end of the time period $2 \tau$. That is, the chemical shift is refocused and does not create any modulation of peak heights as a function of the refocusing time $2 \tau$. If the refocusing period is used as an evolution time, there will be no modulation component due to chemical shift, and chemical shift will not be a measurable frequency in the new $\left(\mathrm{F}_{1}\right)$ dimension. A modulation in detected signal is introduced when two spins are coupled and both experience a $180^{\circ}$ pulse.

This can be explained using two $J$-coupled spin vectors (A and X). The coupling to the $\mathrm{X}$ spin splits the natural frequency of A into two values, separated by $J \mathrm{~Hz}$, the coupling constant. For this on resonance condition, one A component will precess with a frequency $+J / 2$ denoted by $\mathrm{F}$, the other frequency $-J / 2$ denoted by $\mathrm{S}$. The $\mathrm{F}$ component has an attached $\mathrm{X}$ spin whose orientation is $\alpha$ and $\mathrm{S}$ component has an attached $\mathrm{X}$ spin whose orientation is $\beta$. Only the positions in the xy plane of A components $F$ and $S$ are affected by the $180^{\circ}$ refocusing pulse. Their precession characteristics are determined solely by the orientations of the attached $\mathrm{X}$ spins. The separate inversion pulse on $\mathrm{X}$ changes the spin states of the $X$ spins: $\alpha_{x}$ turns into $\beta_{x}$ and $\beta_{x}$ turns into $\alpha_{x}$. Those A spins previously attached to $\alpha_{x}$ spins (F spins) are now attached to $\beta_{x}$ spins, and thus become $S$ spins. Those A spins previously attached to $\beta_{\mathrm{x}}$ spins (S spins) are now attached to $\alpha_{\mathrm{x}}$ spins, and thus become $\mathrm{F}$ spins. Therefore, their precession directions reverse and $\mathrm{A}$ spins coupled to $\mathrm{X}$ spins do not refocus. A spins not coupled to $\mathrm{X}$ are unaffected by any $\mathrm{X}$ pulse and proceed in the same precessional sense and ultimately refocus. The change of direction in precession prevents the refocusing after $2 \tau$, and the final orientation of the spin vectors will be a function of $J$ and $2 \tau$. This experiment, if conducted as a function of $2 \tau$ (or $t_{1}$ ), leads to a modulation of the phase of the echo signal. The modulation frequency in this simple system is $J$.

The localized COSY can be converted to a constant time experiment with the elimination of $t_{1}$-dependent homonuclear $J$ modulations. This results in collapse of 
multiplet splittings in the $F_{1}$ domain and allows the individual cross peak multiplets to be more easily distinguished in crowded spectra. $J$ coupling appear in $\mathrm{F}_{2}$ in the usual way. The main feature of a constant-time experiment is that the total time $T_{c t}$ between preparation and mixing pulses is constant for the entire experiment. Chemical shift evolution during this time can be generated by introducing a $180^{\circ}$ pulse whose position is incremented through the period $\mathrm{T}_{\mathrm{ct}}$. In this sequence the $180^{\circ}$ pulse refocuses the chemical shift evolution. Evolution of the homonuclear $\mathrm{J}$ is unaffected by the $180^{\circ}$ pulse and occurs always to the same extent over the complete interval $\mathrm{T}_{\mathrm{ct}}$. Note that in the pulse sequence the $t_{1}$ period is represented by two half periods, each of which must be incremented by half the normal $t_{1}$.

Cross peaks and diagonal peaks show their typical antiphase absorption and inphase dispersion in $F_{2}$, but both types of peaks can be phased to absorption in the decoupled $F_{1}$ domain. The quality of $F_{1}$ decoupling depends on the quality of the $180^{0}$ pulse.

The constant $J$ evolution that occurs in $\mathrm{T}_{\mathrm{ct}}$ results in the usual product of sine and cosine factors, for example, $\cos \left(\pi J_{k l} T_{c t}\right)$, which are now constraints that influence the intensities of multiplets (coherence transfer amplitude) without causing splittings in $F_{1}$. For diagonal peaks the product is over cosine terms for all couplings to spin $k$ : for cross peaks the product includes a sine term for the active coupling and cosine terms for passive couplings. The cross peak intensity function has a null at $T_{c t}=n / J_{k l}$ for the active coupling and $T_{c t}=(2 n+1) /\left(2 J_{k m}\right)$ for all passive couplings. The latter condition for all couplings gives a null for diagonal peaks. The minimum value that can be used for $T_{c t}$ is determined by the total number of $t_{1}$ increments required for the desired spectral resolution along $F_{1}$ dimension.

\subsection{Implementation of sequence for a GE $3 T$ MRI/MRS scanner}

The Localized Constant Time COrrelated SpectroscopY (CT-COSY) pulse sequence was designed and implemented in EPIC (Environment for Pulse Programming in C). The sequence uses a combination of three slice-selective RF pulses $\left(90^{0}-180^{\circ}-90^{0}\right)$ for volume localization. The water suppression and outer volume saturation modules are provided with GE 3T Horizon LX scanner. FIG. 4.2 shows the pulse sequence for the 2D 
Localized Constant-Time Correlated technique. Numerically optimized Shinnar-Le Roux (SLR) [45] RF pulses with durations of 3.6, 5.2, and $3.6 \mathrm{~ms}$, respectively are used.

Optimized Shinnar-Le Roux (SLR) pulses have very good slice profiles.

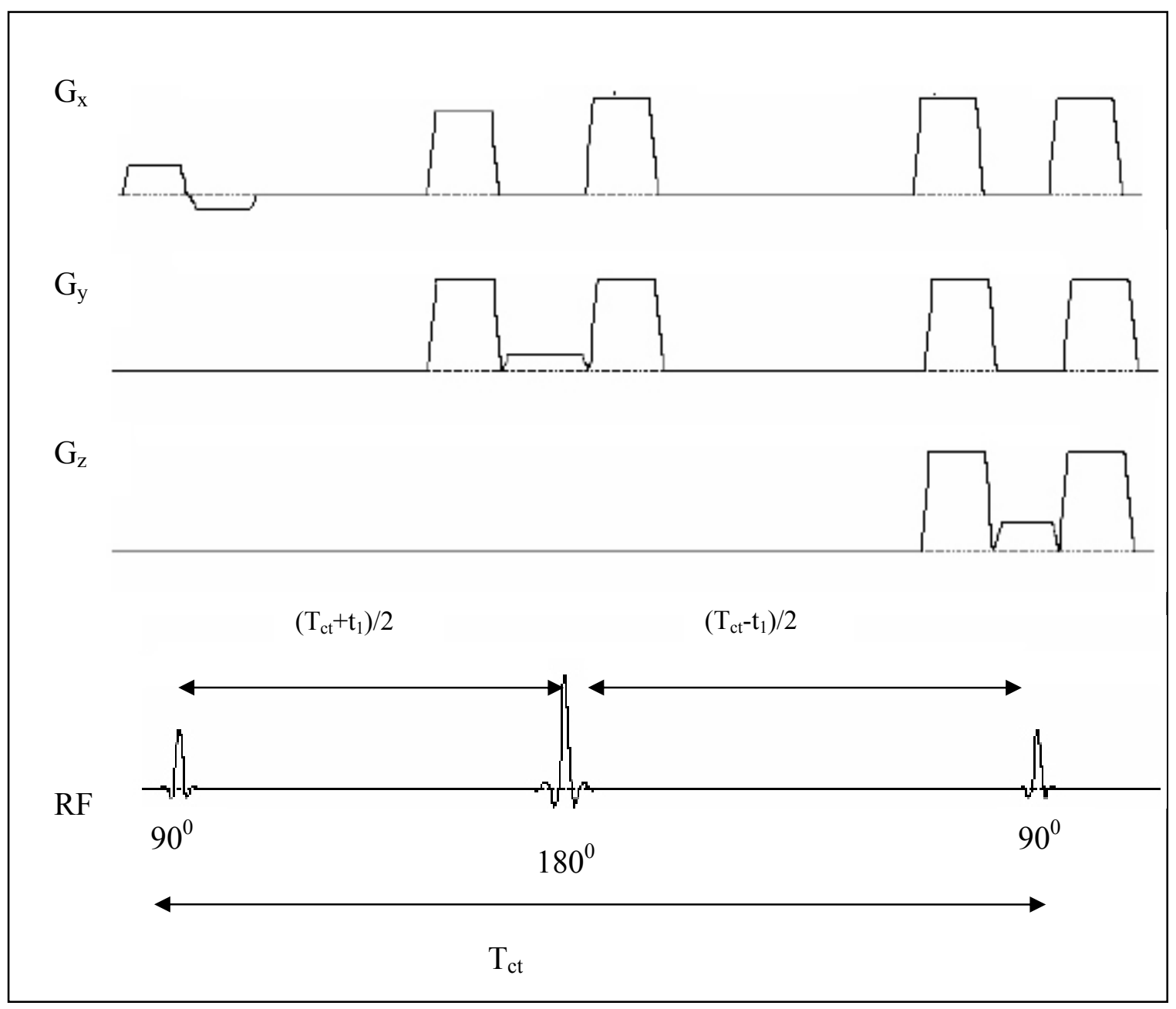

FIG. 4.2 2D Localized Constant-Time Correlated Pulse Sequence

Two incremental delays were introduced symmetric to the $180^{\circ}$ pulse. Now there are two variable time delays. The first delay is between the first $90^{\circ}$ pulse and the $180^{\circ}$ pulse is given by $\left(\mathrm{T}_{\mathrm{ct}}+\mathrm{t}_{1}\right) / 2$. The second delay is between the $180^{\circ}$ pulse and the second $90^{0}$ pulse is given by $\left(\mathrm{T}_{\mathrm{ct}} \mathrm{t}_{1}\right) / 2$. As the $t_{1}$ is incremented the first delay is incremented, the second delay is decremented. So, the time between the start of the first $90^{\circ}$ pulse and acquisition, $T_{\text {ct }}$ is kept constant. This ensures the same echo time and repetition time for each increment. The constant time between the first $90^{\circ}$ pulse and acquisition, $\mathrm{T}_{\mathrm{ct}}$ also 
permits the same amount of coherence transfer for each increment unlike in L-COSY experiment where coherence transfer and $T_{1}, T_{2}$ relaxations vary for each increment as discussed in section 4.0.1. The position of the $180^{\circ}$ pulse is incremented through the period $T_{\text {ct. }}$ In the pulse sequence the $t_{1}$ period is represented by two half periods, each of which must be incremented by half the normal $\Delta \mathrm{t}_{1}$. There are refocusing $\mathrm{B}_{0}$ gradient crusher pulses before and after both the $180^{\circ} \mathrm{RF}$ pulse and the last $90^{\circ} \mathrm{RF}$ pulse.

The central $180^{\circ}$ pulse refocuses chemical shifts, but coupling remains throughout the constant time. The time period $\mathrm{T}_{\mathrm{ct}}$ can be chosen to maximize coherence transfer at all $t_{1}$ increments and minimize the diagonal or cross peaks. This permits to resolve the overlapping peaks. Furthermore, the peaks (diagonal or cross) are decoupled in $t_{1}$ since there is no $J$ modulation with $\mathrm{t}_{1}$. The proposed sequence can be considered as a singleshot technique in terms of volume localization and coherence transfer.

\subsection{Environment for Pulse Programming in C (EPIC)}

EPIC is an acronym that stands for Environment for Pulse programming In $\mathbf{C}$ [29]. EPIC is a programming language, a development environment, and a runtime environment for MR pulse sequences. An EPIC program is executed on a MR Scanner, and controls system hardware to generate a pulse sequence.

\subsubsection{Hardware Overview}

The computer hardware involved in the EPIC environment consists of the host computer (an SGI Indigo2) connected to the TPS (Transceiver Processing and Storage) chassis, which consists of the IPG (Integrated Pulse Generator), the CERD (Combined Exciter Receiver DAB), the ReflexAP (Bulk Access Memory and Array Processor), and the TPS CPU (FIG. 4.3). The IPG is the heart of the pulse generation. 


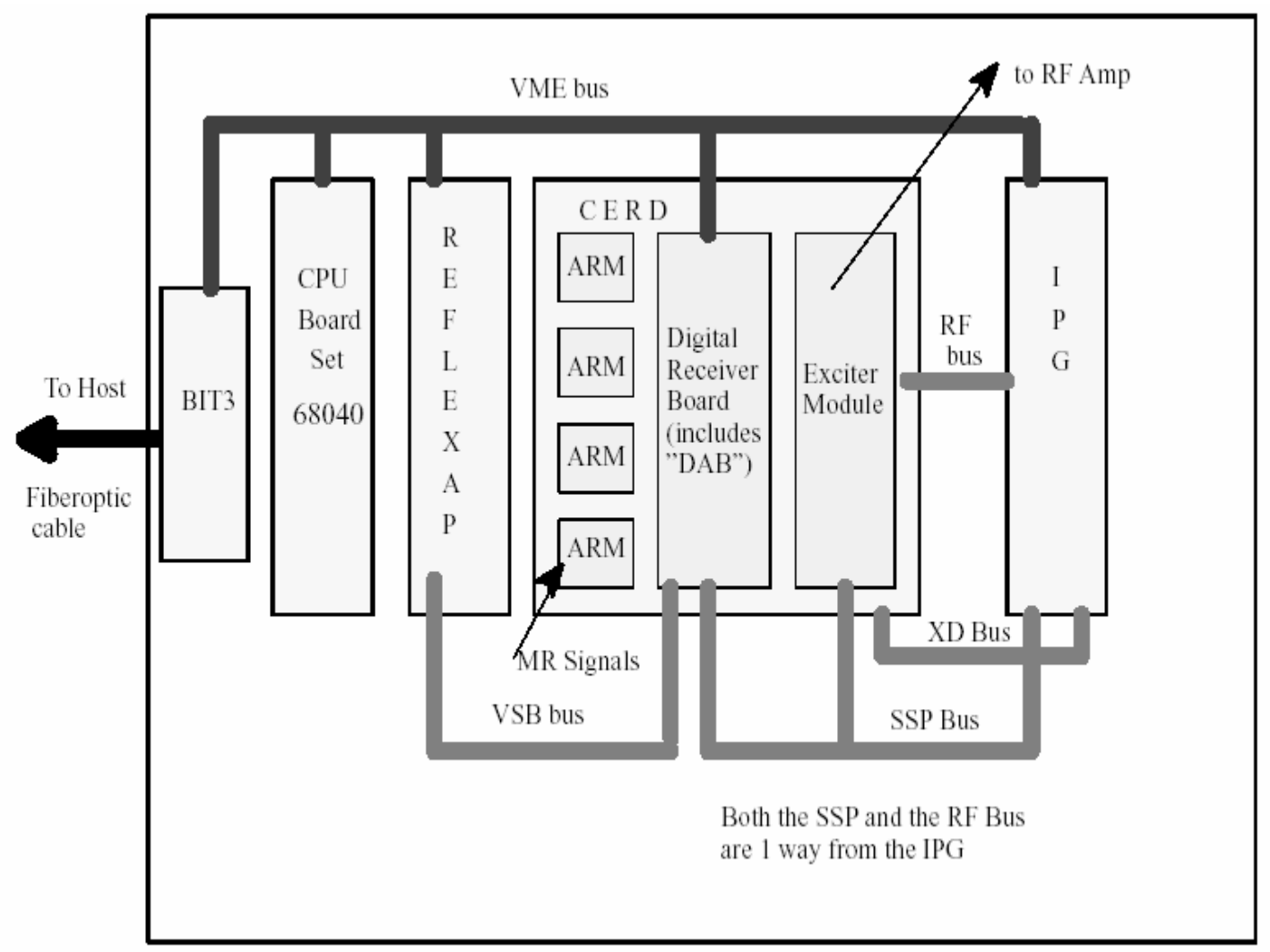

FIG. 4.3 TPS CHASSIS [29]

In order to understand how pulses are generated, one must first understand the concepts of waveform memory, instruction memory and the function of the LCA (Logic Cell Array) based sequencers. The LCA based sequencers are used to produce 16 bit digital waveforms for the gradient and RF amplifiers and to provide control of the devices connected to the SSP (Sequence Synchronous Protocol) bus. There are 8 sequencers XGRAD, YGRAD, ZGRAD, RHO1, RHO2, THETA, OMEGA and SSP and their names indicate which device they control.

The essential purpose of the CERD is to demodulate and sample the MR signal, filter the digitized signal, and scale, sort, average and store the data for subsequent reconstruction processing. The CERD board contains four ARMs (Analog Receive Module), and combines the functionality, which existed on three separate VME boards (Exciter, Receiver, and DAB) in Signa 5.x.

After data acquisition is finished, RECON begins processing raw MR data stored in Bulk Access Memory (BAM), which is a part of the ReflexAP board. The data is processed on 
the AP within the ReflexAP board and then placed in an image work area within the BAM. The TPS is capable of concurrently running data acquisition and reconstruction. Therefore, the operator can begin scanning while images are being reconstructed from the previous scan.

\subsubsection{Software Overview}

The process of compiling and linking the software produces two executable files, namely PSD host process and PSD IPG process. The PSD host process is responsible for turning the operator-entered protocol information (TE, TR, FOV etc.) into pulse parameters (pulse widths, amplitudes, delays etc.), which are used by the second executable. The PSD IPG process is responsible for turning the pulse parameters into a digital representation of a pulse sequence. This process also makes necessary real time modifications as the sequence is played out by the hardware.

The software written by the EPIC programmer is preprocessed and split into 2 source modules. For example, if the EPIC source code is named psd.e (.e being a required extension), the resultant $\mathrm{C}$ sources will be psd.host.c for the host processor and psd.ipg.c for the IPG processor. The EPIC language provides compiler controls to facilitate in this splitting and to allow variables defined in the host section of code to be referenced in the IPG section.

\subsubsection{Communication in EPIC}

The PSD host process communicates with the rest of the Signa system through a set of predefined variables called Control Variables or CVs. FIG. 4.4 shows how the CV is set and it can be noted that the communication is bidirectional. 


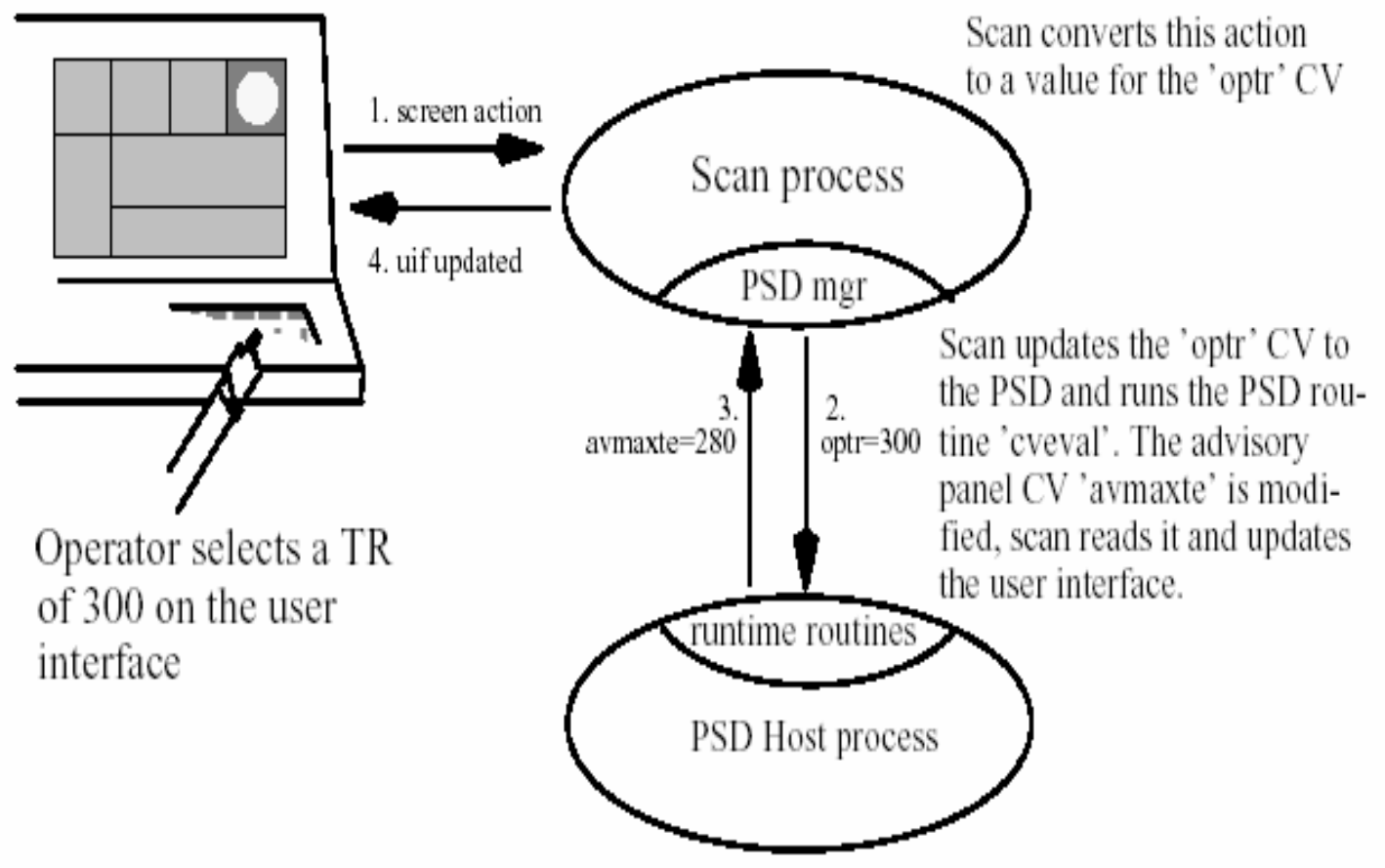

FIG. 4.4 Interprocess Communication[29]

When the operator selects CV (TR) of 300 on the user interface, scan process updates the 'optr' CV value to the PSD and runs the PSD routine 'cveval'. Then the advisory panel $\mathrm{CV}$ 'avmaxte' is modified, scan reads it and updates the user interface.

The Scan, PSD Host and PSD IPG processes are compiled with a 'core' CV include file to allow the PSD manager to communicate the prescription via predefined variables. It is possible to add CVs or 'export' variables whose values are calculated by the PSD Host process and sent to the PSD IPG process. The complete set of CVs and a special set of IPG export variables are sent to the PSD IPG process to communicate pulse widths, start times and scan features. This information is used to generate the data for the hardware sequencers and to control the real time modification and execution of the scan. The PSD IPG process also receives scan information from the rest of the Signa system through the set of CVs and export variables. The PSD IPG process can send information concerning the data about to be collected to the data acquisition software via the SSP bus. 


\subsection{Safety Issues in clinical MRS:}

All MRI scans deposit energy into the patient (or phantom) that occupies the bore of the scanner. In SIGNA the rate of the power deposition is estimated by the PSD with the Signal Absorption Rate (SAR) calculations.

Specific Absorption Rate (SAR) is calculated in the following way:

$S A R \alpha$ (Number of identical RF pulses in sequence) $x$ (Energy deposited by each RF pulse)

(Sequence repetition time) $x$ (Patient Weight)

This shows the strong relationship between the RF pulses, TR times, patient weight and SAR. Product PSDs use a variety of RF pulses with different transmit and/or receive coils, so the RF pulses are not identical nor is energy deposition independent of scan prescription parameters.

This can be resolved by converting the RF pulses used into an equivalent number of standard RF pulses. Then the energy deposited by each standard RF pulse is computed based on parameters in the scan prescription. With these conversions, the SAR calculation becomes:

Average $=($ Number of standard RF pulses in sequence $) \times($ Energy deposited by standard RFpulse $)$ SAR

(Sequence repetition time) $\mathrm{x}$ (Patient Weight)

First the peak B1 of the RF pulse, is calculated with the equation:

$$
\text { peak } B_{1}=\max _{-} b 1 \times \frac{n o m_{-} p w}{p w} \times \frac{a c t \_f a}{n o m_{-} f a}
$$

Since the energy deposited by an RF pulse is proportional to the pulse width and the square of the amplitude, the number of standard pulses that deposit the energy of a specific RF pulse type in a sequence is:

\# Standard Pulses $=$ num $\mathrm{x}$ effwidth $\mathrm{x} \frac{\mathrm{pw}}{\text { standard pulses width }} \times\left[\frac{\text { peak } B_{1}}{\mathrm{~B} 1 \text { standard pulse }}\right]^{2}$

Thus every RF pulse of the sequence gets converted to an equivalent number of standard pulses. Then the sum of all the standard pulses is taken and the average SAR for the sequence is calculated: 
Average $=($ Number of standard RF pulses in sequence $) \mathrm{x}$ (Energy deposited by standard RFpulse $)$ SAR

From EQ. 4.5 it can be seen that the average SAR depends on the number of RF pulses in the sequence and energy deposited by each RF pulse. Our sequence CT-COSY was compiled considering all the regulations including the SAR limits. 


\section{Chapter 5}

\subsection{Results:}

We have demonstrated our localized constant-time two-dimensional correlated MRS technique on a two spin-1/2 system with a J coupling.

\subsubsection{Phantom Preparation}

Trans-cinnamic acid was obtained from Sigma-Aldrich (St. Louis) and was prepared in a $250 \mathrm{ml}$ spherical flask. $100 \mathrm{mM}$ of trans-cinnamic acid was prepared in Methylene Chloride $\left(\mathrm{CH}_{2} \mathrm{Cl}_{2}\right)$. The structure of this molecule is shown in FIG. 5.1.

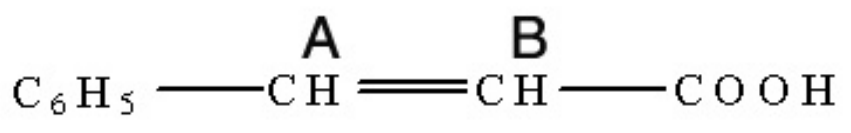

\section{FIG. 5.1 Chemical structure of trans-cinnamic acid}

This molecule includes a two spin $1 / 2$ system (A and B) as shown in the FIG. 5.1, with chemical shifts of 6.5 and $7.8 \mathrm{ppm}$ and coupling constant of $J=16.1 \mathrm{~Hz}$.

\subsection{Optimization}

\subsubsection{Optimization of solvent suppression}

The solvent was suppressed using the water suppression module of our sequence. The solvent signal was selectively excited using chemical shift selective pulses followed by dephasing with gradients. This module is described in section 2.4.3 in chapter 2. The suppression was optimized by adjusting the flip angles of the three solvent selective pulses. The gradient that follow each RF pulse are placed on two axes at a time, with the first pair on the $x$ and $y$ axes, the second on the $y$ and $z$ axes, and the third on the $x$ and $z$. This gradient combination ensures maximum dephasing of the solvent signal.

\subsubsection{Shimming}

Homogeneity of the magnetic field is a major concern in MRS experiments. This is performed by adjusting the currents in the shim coils which corrects for the magnetic field inhomogeniety across the volume from which the signal is observed. Linear and 
second order shims were optimized to achieve maximum suppression of the solvent signal and to improve the line-widths of the observed signal.

\subsection{Scan}

After optimization of the shims we acquire fast gradient echo images of the entire phantom. Gradient echo images (5mm thick) were obtained using a flip angle of $20^{\circ}$, repetition time of $30 \mathrm{~ms}$, field of view of $12 \mathrm{~cm}$ (read) and $12 \mathrm{~cm}$ (phase) dimensions with 256 points along both directions resulting in a spatial resolution of $468 \mu \mathrm{m} \times 468 \mu \mathrm{m}$. A voxel is selected using the graphical interface of GE software. An extremity coil was employed in transmit-receive mode for all the MRS experiments.

\subsubsection{Experimental parameters}

The two-dimensional localized correlated MRS (L-COSY) experiment described in chapter 1 and CT-COSY experiments were performed with the following parameters. Voxel size $27 \mathrm{ml}\left(3 \times 3 \times 3 \mathrm{~cm}^{3}\right)$, time of repetition $(\mathrm{TR})=2000 \mathrm{~ms}$, echo time $(\mathrm{TE})=30 \mathrm{~ms}$, and eight averages per $t_{1}$ increment was performed. The variable incrementation period was set to $800 \mu \mathrm{s}$. We employed 2048 complex points along $\mathrm{F}_{2}$ (acquisition dimension) and 64 points along $\mathrm{F}_{1}$ dimension.

\subsection{Post Processing of the Data}

\subsubsection{Apodization}

Apodization is the multiplication of an FID by a window function. The purpose of apodization is to modify the final spectrum in such a way that certain parameters can be measured more easily. Usually, either spectral signal-to-noise or resolution is enhanced at the expense of degradation of the other characteristic. As a general rule, sensitivity is increased by enhancing the beginning of the FID (where signal-to-noise is highest) and resolution is enhanced by suppressing the beginning of the FID. A number of window functions can be used for sensitivity enhancement such as Linear apodization EQ. (5.1), trapezoidal multiplication EQ. (5.2), and sinebells EQ. (5.3) where $a, b, n, t_{1}, t_{2}$ and $t_{3}$ are apodization parameters, $t$ is time, and $X^{\prime}(t)$ is the apodized FID. 
Linear: $X^{\prime}(t)=X(t)(1-a t), \quad 0<t<1 / a$

Trapezoidal: $\quad X^{\prime}(t)=X(t) t / t_{1}, \quad 0<t<t_{1}$ :

$$
\begin{aligned}
& X(t), t_{1} \leq t \leq t_{2} ; \\
& X(t)\left(t_{3}-t\right) /\left(t_{3}-t_{2}\right), \quad t_{2}<t<t_{3}
\end{aligned}
$$

Sinebell: $\quad X^{\prime}(t)=X(t) \sin ^{n}(a t+b)$

\subsubsection{Zero Filling}

One technique used to increase spectral resolution is zero filling. Zero's are added to the end of the FID so that when the Fourier transform is carried out, more data points describe the spectrum. Independent information can be obtained by zero filling and the resolution is proportional to $n \sin (\pi / n) / \pi$ where $n$ is the ratio of the number of points in the spectrum to the number of time domain points. After one zero fill, the resolution is $90 \%$ of the maximum.

\subsubsection{Linear Prediction}

Linear predictive analysis is a non-iterative method that fits a number of decaying sinusoids to a FID in the presence of noise. The concept behind linear prediction is that each data point $\left(x_{n}\right)$ can be expressed as linear combination of $L$ previous points

$$
x_{n}=\sum_{r=1}^{L} p_{r} x_{n-r}
$$

where $p_{r}$ is a linear prediction parameter. It can be shown that for $x_{n}=\exp [(2 \pi(f-k) n \Delta t+\gamma)]$, that $p_{1}=2 \exp [(2 \pi f-k) \Delta t]$ and $p_{2}=-\exp (-2 k \Delta t)$ where $\mathrm{f}$ is frequency, $\mathrm{k}$ is decay constant, $\Delta \mathrm{t}$ is the time interval between points and $\gamma$ is the phase. It can be further shown that if $x_{n}$ consists of a sum damped sinusoids the $L \geq 2 K$. In general, $L$ is chosen to be about $\mathrm{N} / 4$ where $\mathrm{N}$ is the number of data points. As long as $2 K \leq L \leq N / 2$ the equations may be solved and any excess solutions correspond to noise. The solutions of the polynomial of order $L$, from which decay constants, intensities, frequencies, and phases are determined, are then in order of significance. 
The two-dimensional raw data obtained form the scanner is post processed using commercial software called Felix. A ' $\mathrm{C}$ ' program was employed to convert the data format of GE into Felix format. The $64 t_{1}$ data points were linear predicted to 128 points and zero-filled to 256 points. The 2048 points were zero-filled to 4096 complex points. A squared sine-bell filter was employed on both dimensions and Fourier transformed to yield the spectrum shown in FIG. 5.2.

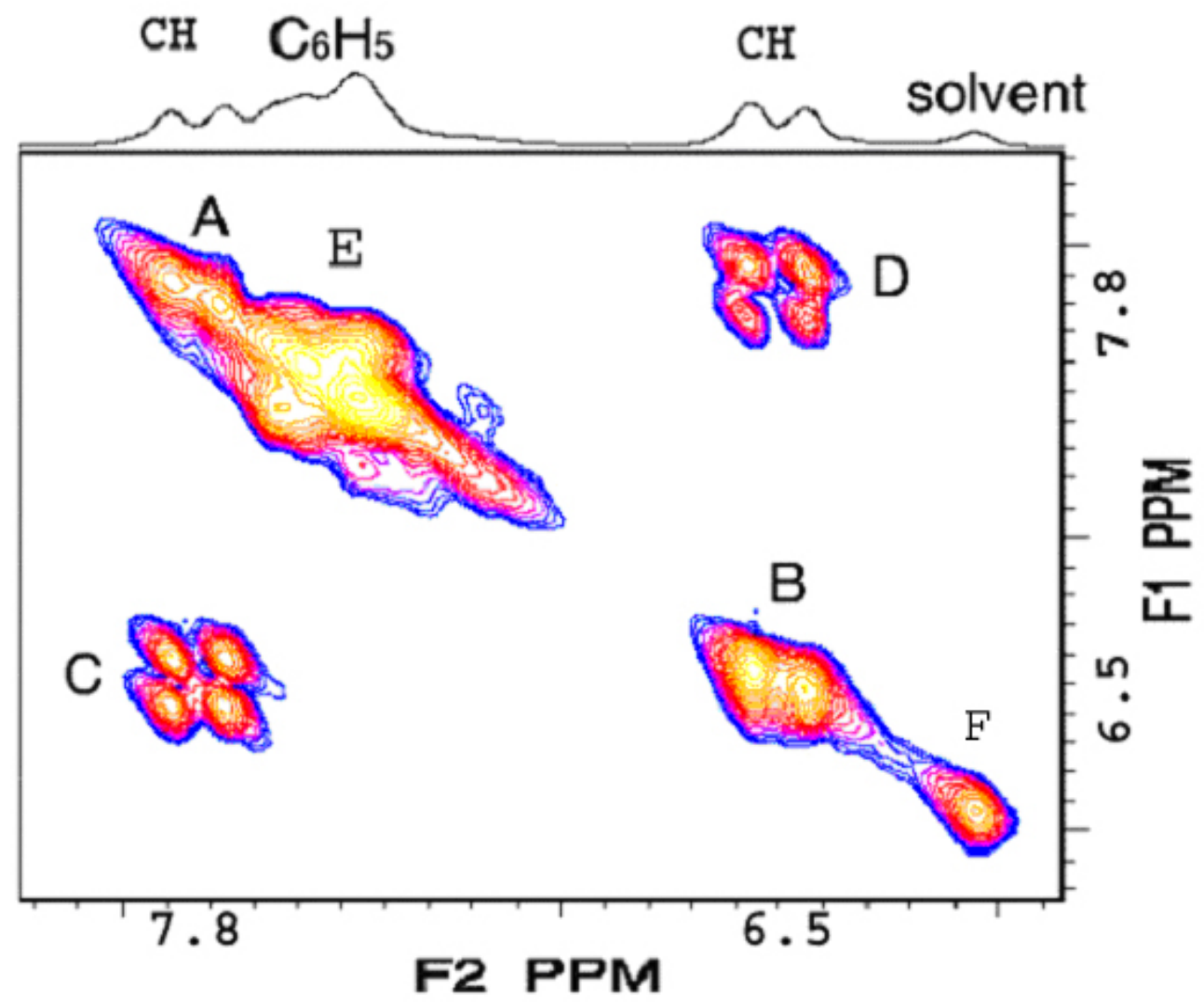

FIG. 5.2 2D L-COSY MR Spectrum of trans-cinnamic acid

We observe the diagonal peaks due to the protons $\mathrm{A}$ and $\mathrm{B}$ and also $\mathrm{E}$ due to the protons from the $\mathrm{C}_{6} \mathrm{H}_{5}$. The diagonal peak $\mathrm{F}$ is due to the solvent used. The cross peaks between $\mathrm{A}$ and $\mathrm{B}$ protons are shown as $\mathrm{C}$ and D in the FIG. 5.2. We can clearly see that the cross 
peaks and diagonal peaks exhibit two doublets along $\mathrm{F}_{1}$ and $\mathrm{F}_{2}$ dimensions with a $J$ splitting of $16.1 \mathrm{~Hz}$.

Theoretical simulation of the 2D COSY spectrum was performed for A and $\mathrm{B}$ protons of trans-cinnamic acid (FIG. 5.3) using 512 acquisition points along $t_{1}$ and $t_{2}$ axes with identical chemical shifts and spin-spin coupling as observed in the experimental spectrum. FIG. 5.3 displays identical splitting pattern as observed in the experimental spectrum. In the theoretical simulations we have increased the spectral resolution in order to observe the splitting on both diagonal and cross peaks. The diagonal (A, B) and cross peaks $(C, D)$ clearly demonstrate the J splitting of $16.1 \mathrm{~Hz}$.

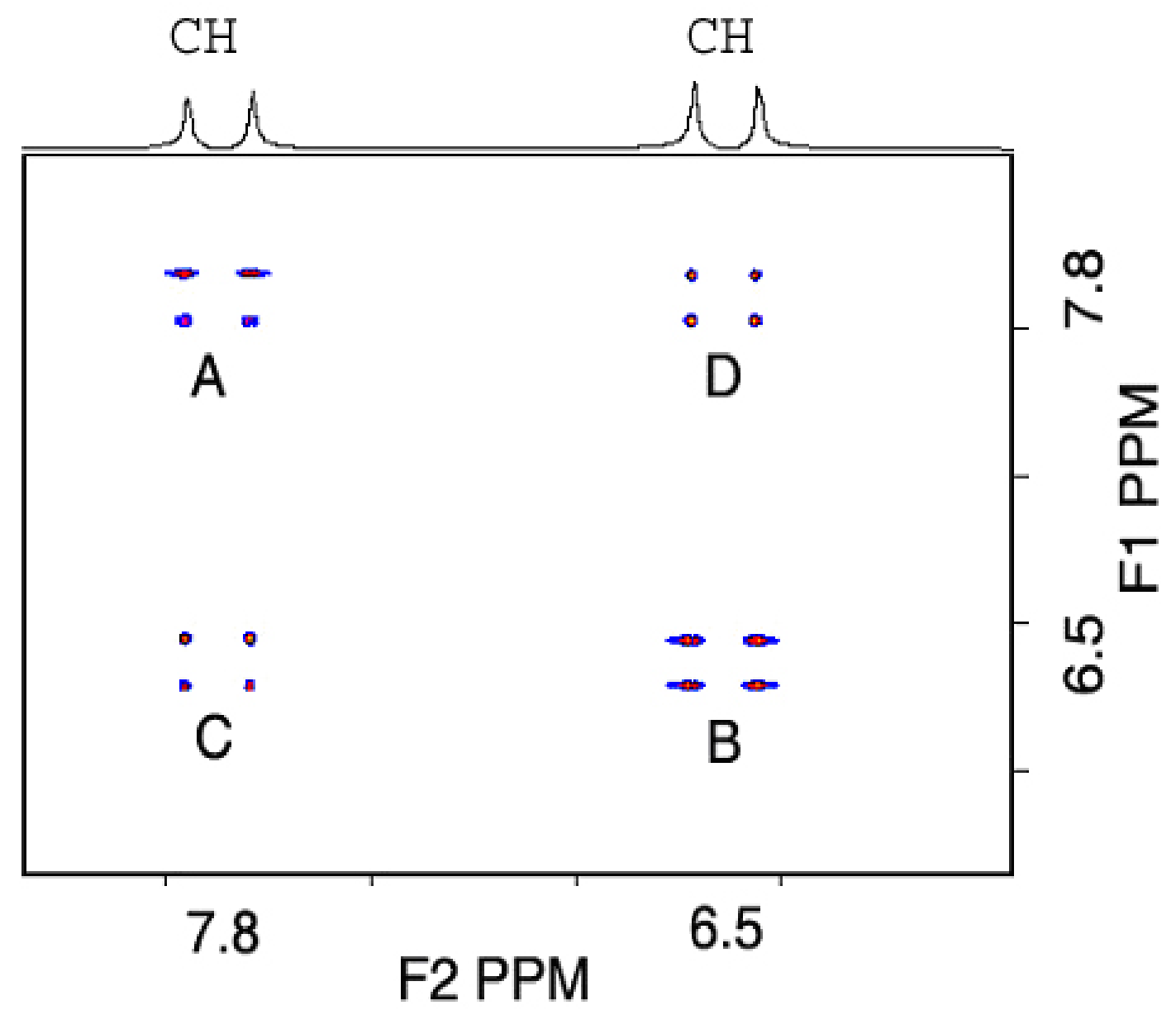

FIG. 5.3 Simulated 2D COSY MR Spectrum of trans-cinnamic acid 


\subsection{Experimental results:}

All the CT-COSY experiments were performed on the identical voxel $(27 \mathrm{ml})$ using the same parameters as employed for the L-COSY experiment. Voxel size $27 \mathrm{ml}$ $\left(3 \times 3 \times 3 \mathrm{~cm}^{3}\right)$, time of repetition $(\mathrm{TR})=2000 \mathrm{~ms}$, and eight averages per $\mathrm{t}_{1}$ increment were performed. In this experiment we have two incrementable delays and they were set to 400 $\mu$ s so that the total incrementation time becomes $800 \mu$ s as employed for the L-COSY experiments. We employed 2048 complex points along $\mathrm{F}_{2}$ (acquisition dimension) and 64 points along $\mathrm{F}_{1}$ dimension. Identical post-processing as employed for L-COSY was performed on the CT-COSY data. The $64 \mathrm{t}_{1}$ data points were linear predicted to 128 points and zero-filled to 256 points. The 2048 points were zero-filled to 4096 complex points. A squared sin-bell filter was employed on both dimensions and then Fourier transformed.

The theoretical amplitude for the cross peaks and diagonal peaks for the A and B spins of trans-cinnamic acid in the CT-COSY experiment can be expressed as:

$$
A_{x} \cos \left(\pi J t_{1}\right) \sin \left(\omega_{A} t_{1}\right)-2 A_{z} B_{y} \sin \left(\pi J t_{1}\right) \sin \left(\omega_{x} t_{1}\right)
$$

The diagonal peak intensity is proportional to $\cos \left(\pi J t_{1}\right)$ and cross peak intensity to $\sin \left(\pi J t_{1}\right)$. The time period $t_{l}$ can be chosen to maximize coherence transfer to either diagonal peaks or cross peaks. When constant time is optimized for $T_{\text {ct }}=1 / 2 J$ the coherence transfer to cross peaks gets maximized. When constant time is optimized for $\mathrm{T}_{\mathrm{ct}}=1 / J$ the coherence transfer to diagonal peaks gets maximized.

FIG. 4.3 shows the CT-COSY spectrum for $\mathrm{T}_{\text {ct }}$ value corresponding to $3 / 2 J(93 \mathrm{~ms})$ where $J=16.1 \mathrm{~Hz}$. The coherence transfer to cross peaks gets maximized according to EQ. 5.5. Note the collapse of the doublets along the $F_{1}$ dimension shown in FIG. 5.4 due to constant time evolution. 


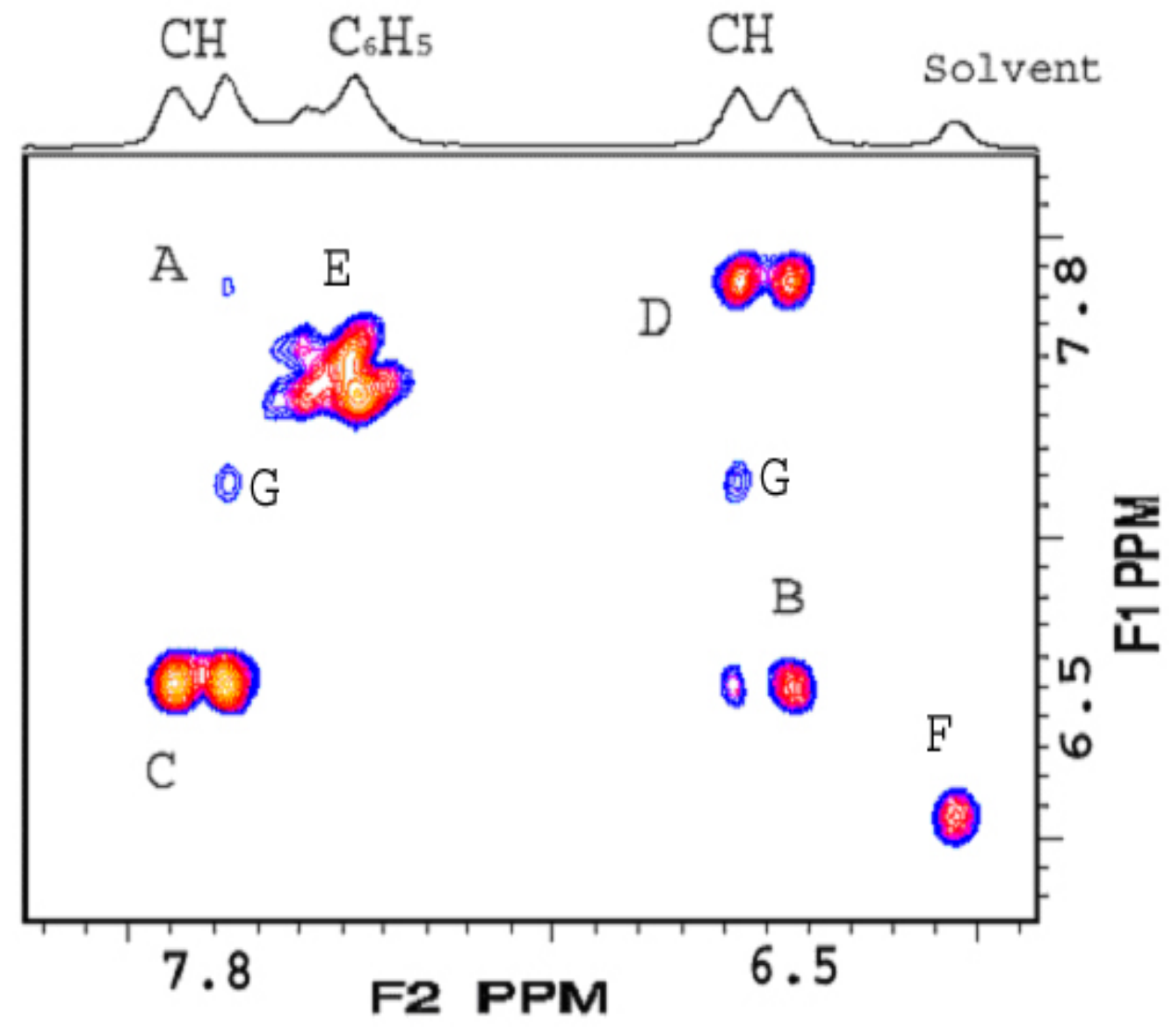

FIG. 5.4 2D CT-COSY Spectrum $\left(T_{c t}=3 / 2 J(93 m s)\right)$

FIG. 5.4 shows the simulated spectrum of CT-COSY for the two protons A and B of trans-cinnamic acid with identical $T_{c t}$ time of $93 \mathrm{~ms}\left(T_{\mathrm{ct}}=3 / 2 \mathrm{~J}\right)$. We clearly see the maximum coherence transfer to the cross peaks. A, B are diagonal peaks from the protons, $\mathrm{E}$ is due to $\mathrm{C}_{6} \mathrm{H}_{5}$ group and $\mathrm{F}$ is due to the solvent used. $\mathrm{G}$ is the artifactual peak at the center frequency of the spectrum which arises due to the strong coupling effects (trans-cinnamic acid has coupling of $16.1 \mathrm{~Hz}$ ). 
Theoretical simulations were performed for A and B protons of trans-cinnamic acid.

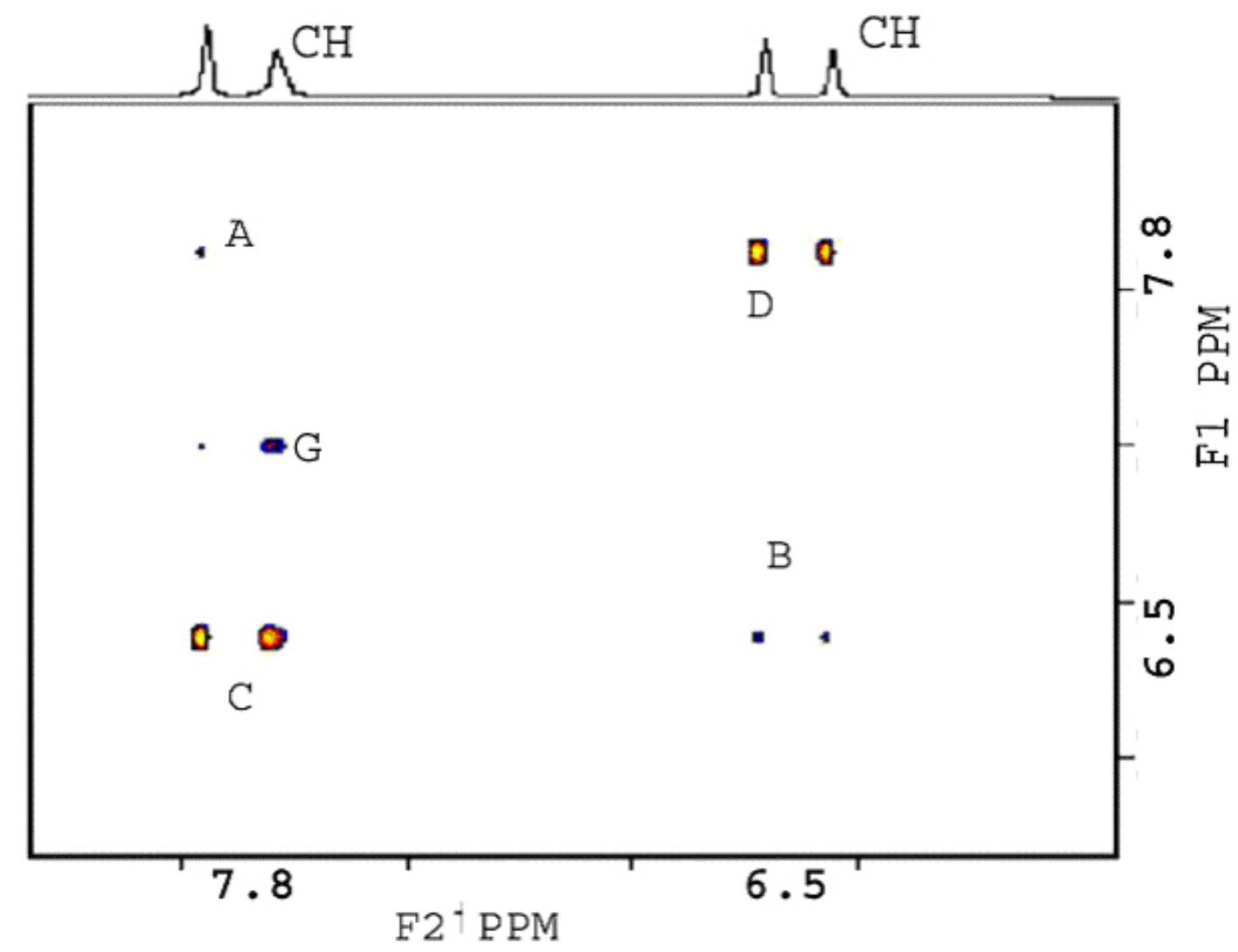

FIG. 5.5 Simulated spectrum of CT-COSY sequence. Coherence transfer to cross peaks is maximized.

FIG. 5.5 shows the simulated CT-COSY spectrum. The coherence transfer is maximized for cross peaks (C, D) whereas diagonal peaks (A, B) intensity becomes weak. Constant time $T_{\text {ct }}$ increases in simulations as we consider 256 points along both $F_{1}$ and $F_{2}$ dimensions. The spectrum is clear as only $\mathrm{AB}$ spins are simulated, ignoring other spins. We can also observe the artifactual peak $G$ even in simulation result. 


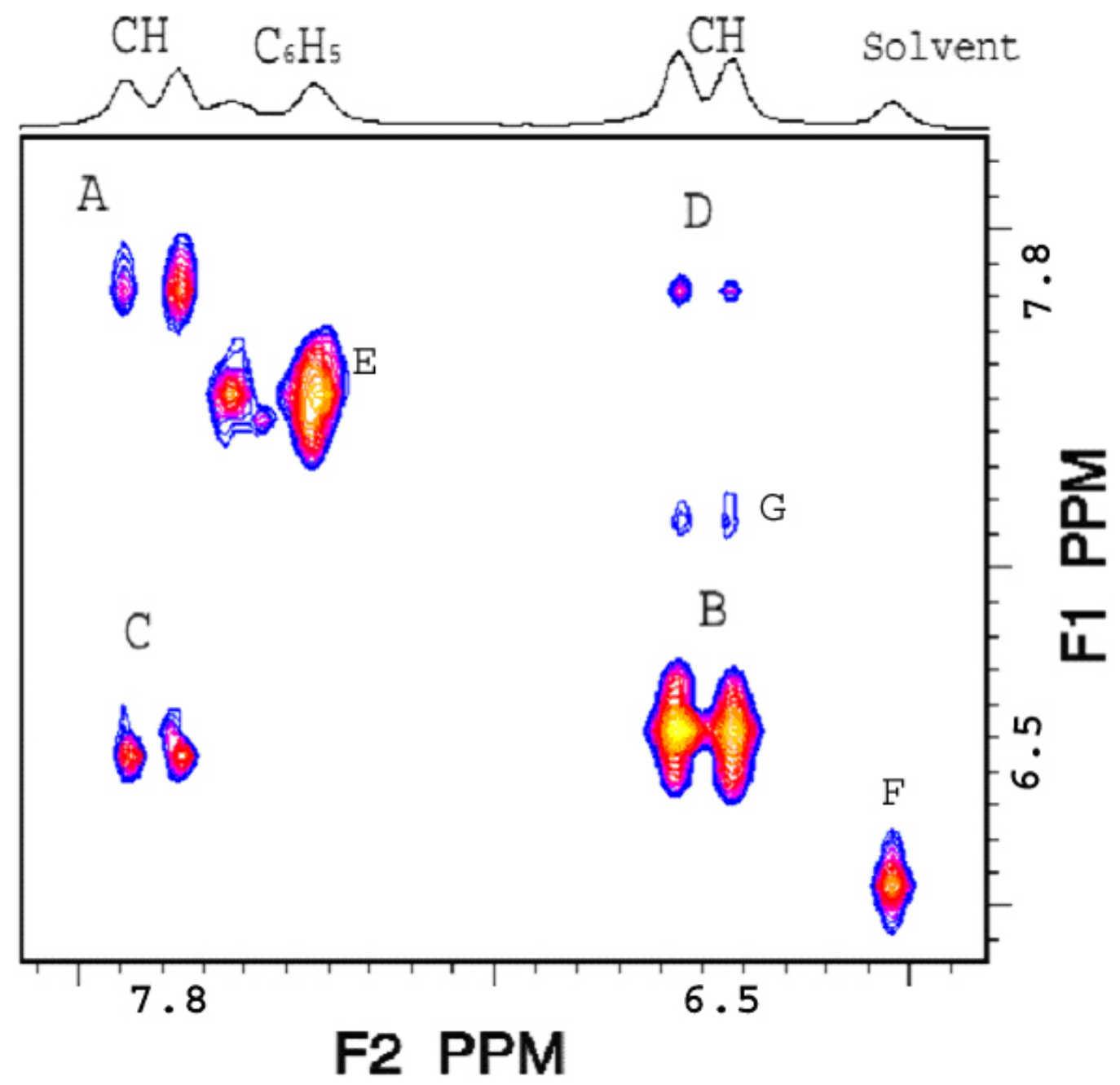

FIG. 5.6 Experimental result of CTCOSY $\left(T_{c t}=2 / J(124 m s)\right)$

FIG. 5.6 shows the experimental result of CTCOSY technique for $\mathrm{T}_{\mathrm{ct}}$ value corresponding to $2 / J(124 \mathrm{~ms})$ where $J=16.1 \mathrm{~Hz}$. The coherence transfer to the diagonal peaks (A, B) gets maximized according to EQ. 5.5. Again note the collapse of the doublets along the $\mathrm{F}_{1}$ dimension due to constant time evolution. The cross peaks $(\mathrm{C}, \mathrm{D})$ intensity is weak. Diagonal peak $\mathrm{E}$ is due to the $\mathrm{C}_{6} \mathrm{H}_{5}$ group in trans-cinnamic acid and peak $F$ is due to the solvent used. $G$ is the arifactual peak. 


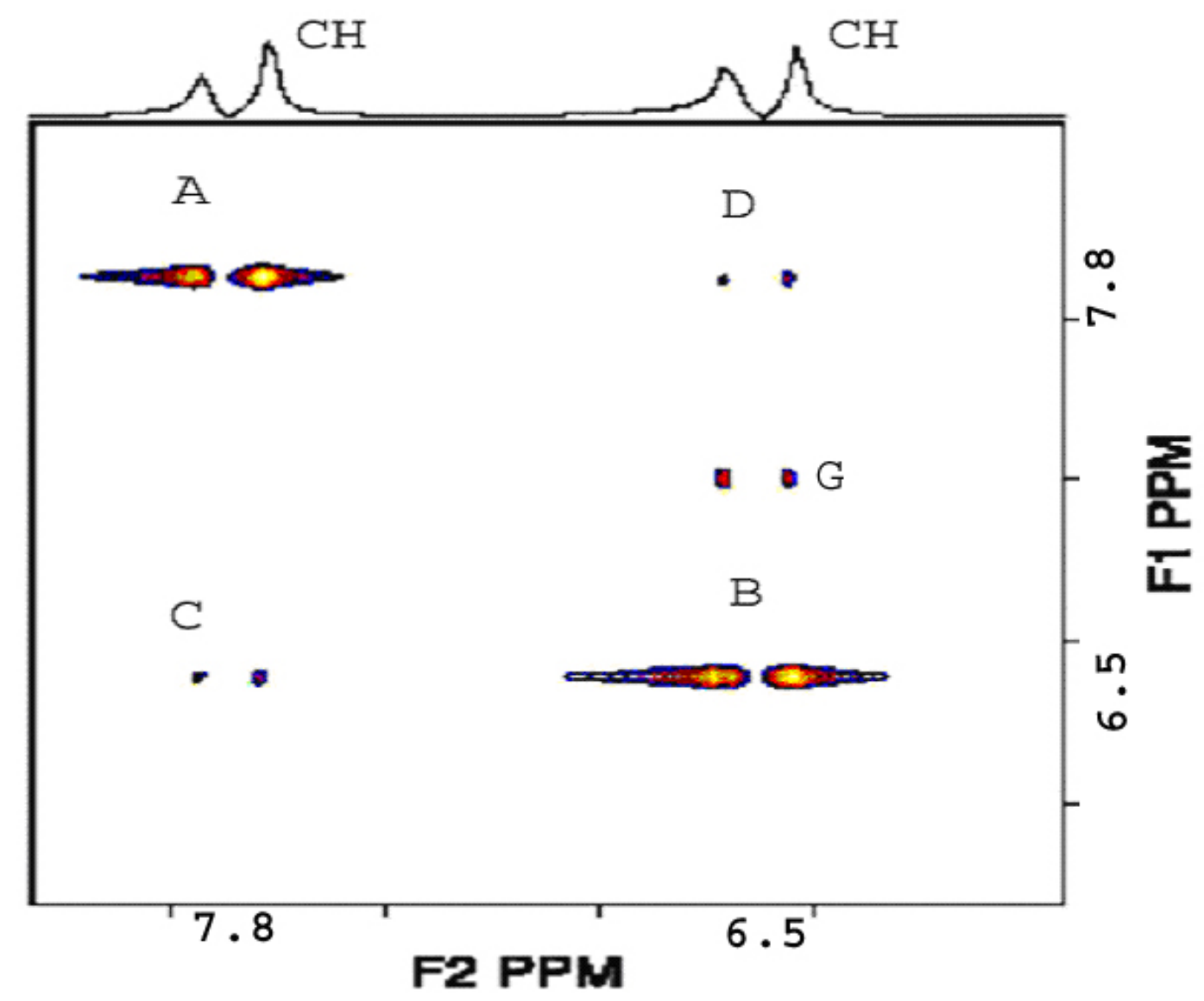

FIG. 5.7 Simulated result of CT-COSY technique. Coherence transfer is maximized to diagonal peaks.

FIG. 5.7 shows the simulated spectrum of of CT-COSY for the two protons A and B of Trans-cinnamic acid. We clearly see the maximum coherence transfer to the diagonal peaks.

We observe artifactual peaks (G) at the center frequency of the spectrum in both cases, experimental and simulations. These artifactual peaks result from the strong coupling effects [46]. Trans-Cinnamic acid has a strong coupling of $16.1 \mathrm{~Hz}$. This leads to the appearance of the artifactual peaks at the center frequency in the spectrum. 


\section{Chapter 6}

\subsection{Conclusions}

In this thesis we have developed a novel localized constant-time correlated MRS technique for a clinical GE 3T MRI/MRS scanner to overcome the following limitations that exist in two-dimensional localized correlated spectroscopy for clinical applications.

1) The variable incrementation period in $2 \mathrm{D}$ L-COSY sequence changes the total echo time for every increment. This result in variable coherence transfers in each experiment and is different for each metabolite with different spin-spin couplings.

2) This sequence also introduces variable $T_{1}$ (Spin-Lattice relaxation) and $T_{2}$ (SpinSpin relaxation) effects on the cross peaks and diagonal peaks which complicates the quantification.

3) The splitting of the cross peaks due to spin-spin coupling reduces the sensitivity resulting in longer scan time and also there is still some overlap of cross peaks at clinical field strengths.

We have addressed the above challenges by designing the localized constant-time correlated spectroscopy (L-COSY) for metabolite characterization and quantification.

In our sequence every $t_{1}$ increment has the same echo time and repetition time resulting in identical $\mathrm{T}_{1}$ and $\mathrm{T}_{2}$ weighting for each scan. Due to the constant time the $J$-decoupling along $F_{1}$ dimension has been achieved.

We have successfully implemented and evaluated our new localized constant-time two-dimensional correlated MRS technique on the GE 3T MRI / MRS scanner. The experimental demonstrations of the CT-COSY technique on a two-spin $1 / 2$ system has been compared with L-COSY technique and theoretical simulations. The coherence transfer to diagonal peaks or cross peaks have been demonstrated by employing suitable constant time periods. The cross peaks and diagonal peaks (doublets) collapse along $\mathrm{F}_{1}$ dimension resulting in enhanced sensitivity. These features of the technique permits absolute quantification of the metabolites as compared to the L-COSY technique where quantification can be achieved only by taking metabolite ratios.

This new localized two-dimensional constant-time technique will permit detection and quantification of J-coupled metabolites with high sensitivity and also in reduced 
experimental time. The potential applications of this technique for in vivo applications include metabolites in the brain, breast and calf-muscle.

\subsection{Future work}

The CT-COSY experiments have to be performed for various metabolites and should be analyzed for coherence transfer to cross peaks and diagonal peaks. This will permit optimization of the sequence for in vivo applications. For example the sequence can be optimized to a specific metabolite with appropriate constant time. This sequence also permits absolute quantification due to constant $T_{1}$ and $T_{2}$ for each $t_{1}$ increment. The collapse of the multiplets in $F_{1}$ dimension increases the sensitivity of the cross peaks as compared to the existing L-COSY technique. The sensitivity of the experiment could be increased by three-to-fivefold since there is no loss of intensity from cancellation in antiphase multiplets in $t_{1}$. However the increased duration of constant-time introduces larger losses due to transverse relaxation as compared to L-COSY technique. These effects have to be optimized by theoretical simulations including transverse relaxation. Our current simulations do not include the losses due to transverse relaxation. 


\section{Bibliography}

[1] E.M.Purcell, H.C.Torrey and R.V.Pound, 'Resonance absorption by nuclear magnetic moments in a solid', Phys.Rev. 69, 37-38 (1946).

[2] F.Bloch,W.W.Hansen and M.E.Packard,'Nuclear induction', Phys.Rev. 69,127(1946)

[3] F.Bloch, 'Nuclear induction', Phys.Rev. 70, 460-473 (1946).

[4] F.Bloch, W.W.Hansen and M.Packard, 'The nuclear induction experiment', Phys.Rev. 70, 474-485 (1946).

[5] R.R.Ernst and W.A.Anderson, 'Applications of Fourier transform spectroscopy to magnetic resonance', Rev.Sci.Instrum. 37, 93-102 (1966).

[6] R.A.Iles, A.N.Stevens and J.R.Griffiths, NMR studies of metabolites in living tissues, Prog.NMR Spectroscopy 15, 49-200 (1982).

[7] M.J.Avison, H.P.hetherington and R.G.Shulman, 'Applications of NMR to studies of tissue metabolism', Ann.Rev.BIophys.Chem.15, 377-402 (1986).

[8] O.A.C.Petroff, 'Biological ${ }^{1}$ H NMR spectroscopy', Comp.Biochem.Physiol. 908, 249-260 (1988).

[9] S.Cerdan and J.Seeing, 'NMR studies of metabolism', Ann.Rev.BIophys.Chem. 19, 43-67 (1990).

[10] B.D.Ross, 'The biochemistry of living tissue:Examination by MRS', NMR Biomed. 5,215-219 (1992).

[11] S.R.Williams, 'In vivo proton spectroscopy.Experimental aspects and potential', In NMR Basic Principles and Progress P.Diehl, E.Fluck,H.Gunther,R.Kosfeld and J.Seeing,(Eds) Vol.28,p.55-71,Springer-Verlag,Berlin (1992).

[12] F.A.Howe, R.J.Maxwell, D.E.Sauders, M.M.Brown and J.R.Griffiths, 'Proton spectroscopy in vivo', Magn.Reson. Q. 9, 31-59 (1993).

[13] H.Bachelard and R.Badar-Goffer, 'NMR spectroscopy in neurochemistry', J.Neurochem. 61,412-429 (1993).

[14] O.Hendriksen, 'MR spectroscopy in clinical research', Acta Radiol. 35, 96-116 (1994).

[15] B.Ross and T.Michaelis, 'Clinical applications of magnetic resonance spectroscopy', Magn.Reson.Q. 10,191-247 (1994). 
[16] W.G.Proctor and F.C.Yu 'The dependence of a nuclear magnetic resonance frequency ', Phys.Rev.77, 717(1950).

[17] W.C.Dickinson, 'Dependence of the F19 nuclear resonance position on chemical compound', Phys. Rev. 77.736 (1950).

[18] N.F.Ransey and E.M.Purcell, 'Interactions between nuclear spins in molecules', Phys.Rev. 85, 143-144 (1952).

[19] Robin.D.Graaf. IN VIVO NMR SPECTROSCOPY: JOHN WILLEY \&SONS: 1998.

[20] E.L.Hahn. 'Spin Echoes', Phys. Rev.94, 630-638 (1954)

[21] William R.Croasmun and Robert M.K.Carlson 'Two-Dimensional NMR Spectroscopy' VCH Publishers. 1994.

[22] James Keeler, Basics of Two Dimensional NMR.

[23] J.H.den Boef, C.M.J.van Uijen and C.E.Holzschere, 'Multiple-slice NMR imaging by three-dimensional Fourier zeugmatography', Phys. Med.biol. I 29,857-862 (1984)

[24] L.E.Crooks,J.Watts, J.Hoenniger,M.Arakawa,L.Kaufman,H.Guenther and D.Feinberg,'Thin-section definition in magnetic resonance imaging:technical concepts and their implementation', Radiology 154, 463-467 (1985).

[25] D.A.Feinberg, L.E.Crooks, J.C.Hoenninger, J.C.Watts, M.arakawa, H.Chang and L.Kaufman, 'Contigous thin multisection MR imaging by two-dimensional Fourier transform techniques', Radiology 159,811-817 (1986)

[26] I.R.Young, D.J.Bryant and J.A.Payne, 'Variation in slice shape and absorption artifacts in the determination of tissue parameters in NMR imaging', Magn.Reson.Med. 2,355-389 (1985).

[27] P.Devolon, L.Emsley,P.Weber, R.Meuli, M.Decorps and G.Bodenhausen, 'Methods for reconstructing phase sensitive slice profiles in magnetic resonance imaging', Magn.Reson.Med. 31,178-183 (1994).

[28] A.Haase, J.Frahm, W.Hanicke and D.Mattaei, " ${ }^{1} \mathrm{H}$ NMR chemical shift selective (CHESS) imaging',Phys.Med.Biol. 30,341-344 (1985).

[29] GE’s EPIC Manual. 
[30] P.A.Bottomley, 'Selective volume method for performing localized NMR spectroscopy', U.S.patent 4480228 (1984).

[31] P.A.Bottomley, 'Spatial localization in NMR spectroscopy in vivo', Ann.N.Y.Acad. Sci. 508, 333-348 (1987).

[32] W.I.Jung, 'Localized double spin echo proton spectroscopy part I: Basic concepts', Concepts.Magn.Reson. 8, 1-17 (1996).

[33] W.I.Jung, 'Localized double spin echo proton spectroscopy part II:Basic concepts', Concepts.Magn.Reson. 8, 77-103 (1996).

[34] J.Frahm, K.D.Merbodlt and W.hanicke, 'Loclaized proton spectroscopy using stimulated echoes', J.Magn.Reson. 72, 502-508 (1987).

[35] J.Granot, 'Selected volume excitation usinf stimulated echoes (VEST).Application to spatially localized spectroscopy and imaging', J.Magn.Reson. 70,488-492 (1986).

[36] R.Kimmich and D. Hoepfel,'Volume -selective multipulse spin-echo spectroscopy', J.Magn.Reson. 72,379-384 (1987).

[37] J.Frahm, H.Bruhn, M.L.Gyngell, K.D.Merbodlt, W.Hanicke and R.Sauter, 'Localized high-resolution proton NMR spectroscopy using stimulated echoes:initial applications to human brain in vivo',Magn.Reson.Med. 9, 79-93 (1989).

[38] C.T.W.Moonen, M.von Kienlin, P.C.M.van Ziji, J.Cohen, J.Gillen, P.Daly and G.Wolf,'Comparision of single-shot localization methods (PRESS and STEAM) for in vivo proton NMR spectroscopy', NMR Biomed. 2,201-208 (1989).

[39] P.C.M.vanZiji, C.T.W.Moonen, J.R.Alger,J.S.Cohen and S.A.Chesnick, 'High field localized proton spectroscopy in small volumes:Greatly improved localization and shimming using shielded strong gradients', Magn.Reson.Med 10, 256-265 (1989).

[40] K.D.Merboldt, D.Chien, W.Hanicke, M.L.Gyngell, H.Bruhn and J.Frahm, 'Localized 13P NMR spectroscopy of the adult human brain in vivo using stimulated-echo (STEAM) sequences', J.Magn.reson. 89, 343-361 (1990).

[41] M. Albert Thomas, Kenneth Yue, Nader Binesh, Pablo Davanzo, Anand Kumar, Benjamin Siegel, Mark Frye, John Curran, Robert Lufkin, Paul Martin, Barry Guze, 'Localized two-dimensional shift correlated MR spectroscopy of human brain', Magn.Reson.Med, 46 ,58-67 (2001).

[42] Smith SA.Levante TO,Meier BH, Ernst RR,'Computer simulations in magnetic resonance.An object oriented programming approach',J.Magn.Reson A106:75105 (1994). 
[43] Govindaraju V, Young K,Maudsley AA,'Proton NMR chemical shifts and coupling constants for brain metabolites.NMR Biomed. 13,129-153 (2000).

[44] David E.Wemmer, 'Homonuclear Correlated Spectroscopy (COSY): The basics of Two-Dimensional NMR', Concepts .Magn.Reson. 1,59-72 (1989).

[45] Antonella Raddi and Uwe Klose,'Optimized Shinnar-Le Roux RF 1800 Pulses in Fast Spin-Echo Measurements', J.Magn.Reson 9,613-620 (1999).

[46] Zhengrong Wu, Ad Bax, 'Measurement of Homonuclear Proton Couplings Based on Cross_Peak Nulling in CT-COSY', J.Magn.Reson 151,242-252 (2001).

[47] N. Chandrakumar and S. Subramanian, 'Modern Techniques in high-resolution FTNMR', Springer-Verlag, 1987. 